\title{
TWO-DIMENSIONAL STEADY SUPERSONIC EXOTHERMICALLY REACTING EULER FLOW PAST LIPSCHITZ BENDING WALLS
}

\author{
GUI-QIANG G. CHEN, JIE KUANG, AND YONGQIAN ZHANG
}

\begin{abstract}
We are concerned with the two-dimensional steady supersonic reacting Euler flow past Lipschitz bending walls that are small perturbations of a convex one, and establish the existence of global entropy solutions when the total variation of both the initial data and the slope of the boundary is sufficiently small. The flow is governed by an ideal polytropic gas and undergoes a one-step exothermic chemical reaction under the reaction rate function that is Lipschtiz and has a positive lower bound. The heat released by the reaction may cause the total variation of the solution to increase along the flow direction. We employ the modified wave-front tracking scheme to construct approximate solutions and develop a Glimm-type functional by incorporating the approximate strong rarefaction waves and Lipschitz bending walls to obtain the uniform bound on the total variation of the approximate solutions. Then we employ this bound to prove the convergence of the approximate solutions to a global entropy solution that contains a strong rarefaction wave generated by the Lipschitz bending wall. In addition, the asymptotic behavior of the entropy solution in the flow direction is also analyzed.
\end{abstract}

\section{INTRODUCTION}

We are concerned with the problem of the two-dimensional steady supersonic exothermically reacting Euler flow past Lipschitz bending walls that are small perturbations of a convex one (see Fig. 1.1). The governing system for steady exothermically reacting flow consists of the Euler equations with the following form:

$$
\left\{\begin{array}{l}
\partial_{x}(\rho u)+\partial_{y}(\rho v)=0 \\
\partial_{x}\left(\rho u^{2}+p\right)+\partial_{y}(\rho u v)=0 \\
\partial_{x}(\rho u v)+\partial_{y}\left(\rho v^{2}+p\right)=0 \\
\partial_{x}((\rho E+p) u)+\partial_{y}((\rho E+p) v)=0 \\
\partial_{x}(\rho u Z)+\partial_{y}(\rho v Z)=-\rho Z \phi(T)
\end{array}\right.
$$

where $(u, v), p, \rho, Z$, and $\phi(T)$ stand for the velocity, pressure, density, fraction of unburned gas in the mixture, and reaction rate, respectively, and

$$
E=\frac{1}{2}\left(u^{2}+v^{2}\right)+e+\tilde{q} Z
$$

denotes the specific total energy with the specific internal energy $e$, and $\tilde{q}$ is the specific binding energy of unburned gas. The other two thermodynamic variables are the

Date: October 1, 2018.

2010 Mathematics Subject Classification. 35B07, 35B20, 35D30, 76J20, 76L99, 76N10.

Key words and phrases. Steady, supersonic, reacting Euler flow, rarefaction wave, wave-front tracking scheme, Lispchitz bending wall, entropy solutions, asymptotic behavior, stability. 
temperature $T$ and entropy $S$ which are defined through thermodynamical relations:

$$
T d S=d e-\frac{p}{\rho^{2}} d \rho .
$$

Then the pressure $p$ and internal energy $e$ can be regarded as functions of $(\rho, S)$ :

$$
p=p(\rho, S), \quad e=e(\rho, S) .
$$

In particular, $\partial_{\rho} p(\rho, S)>0$ and $\partial_{\rho} e(\rho, S)>0$ for $\rho>0$, and $c=\sqrt{\partial_{\rho} p(\rho, S)}$ is called the local sound speed.

For an ideal polytropic gas, the constitutive relations are given by

$$
p=R \rho T, \quad e=c_{v} T, \quad \gamma=1+\frac{R}{c_{v}}>1,
$$

and

$$
p=p(\rho, S)=\kappa \rho^{\gamma} e^{S / c_{v}}, \quad e(\rho, S)=\frac{\kappa}{\gamma-1} \rho^{\gamma-1} e^{S / c_{v}}=\frac{R T}{\gamma-1},
$$

where $R, \kappa, c_{v}$, and $\gamma>1$ are all positive constants. Then the sonic speed is given by $c=\sqrt{\frac{\gamma p}{\rho}}$.

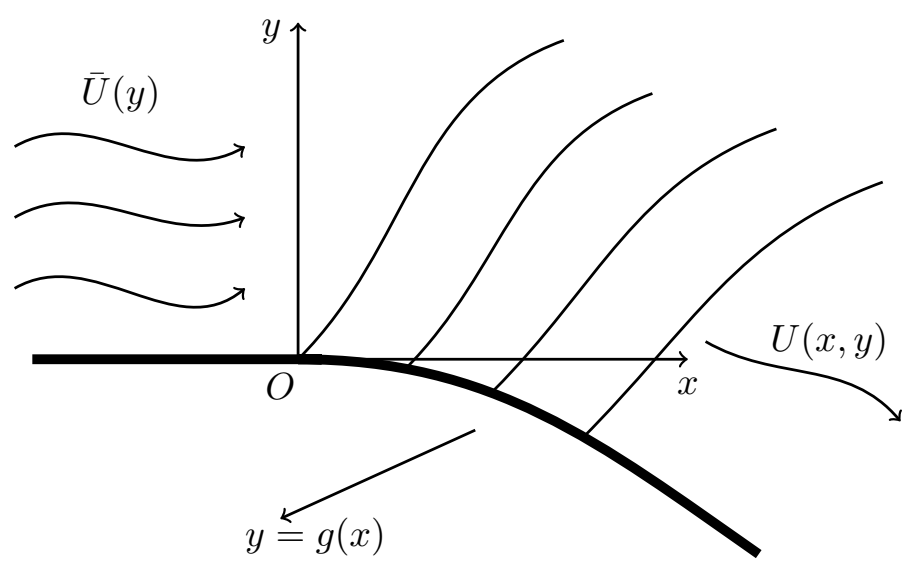

Fig. 1.1. Supersonic reacting flow past a Lipschitz bending wall

In addition, inadmissible discontinuous solutions are eliminated by requiring the following entropy condition for the solutions:

$$
\partial_{x}(\rho u S)+\partial_{y}(\rho v S) \geq \frac{\tilde{q} \rho Z \phi(T)}{T}
$$

in the distributional sense.

System (1.1) for the exothermically reacting steady Euler flow can be written in the following general form:

$$
W(U)_{x}+H(U)_{y}=G(U)
$$


with $U=(u, v, p, \rho, Z)^{\top}$, where

$$
\begin{aligned}
W(U) & =\left(\rho u, \rho u^{2}+p, \rho u v, \rho u\left(\tilde{h}+\frac{u^{2}+v^{2}}{2}\right), \rho u Z\right)^{\top}, \\
H(U) & =\left(\rho v, \rho u v, \rho v^{2}+p, \rho v\left(\tilde{h}+\frac{u^{2}+v^{2}}{2}\right), \rho v Z\right)^{\top}, \\
G(U) & =(0,0,0, \tilde{q} \rho \phi(T) Z,-\rho \phi(T) Z)^{\top},
\end{aligned}
$$

with $\tilde{h}=\frac{\gamma p}{(\gamma-1) \rho}$. When $\rho>0$ and $u>c, U$ can also be represented by $W$, that is, $U=U(W)$, by the implicit function theorem since the Jacobian does not vanish:

$$
\operatorname{det}\left(\nabla_{U} W(U)\right)=-\frac{\rho u^{2}}{\gamma-1}\left(u^{2}-c^{2}\right) \neq 0 .
$$

Throughout this paper, we assume the following:

$\left(\mathbf{H}_{\mathbf{1}}\right)$ The Lipschitz continuous bending wall $y=g(x)$ is a small perturbation of the convex wall $y=g_{*}(x)$ for $x \geq 0$ with

$$
g(x)=0 \quad \text { for } x \leq 0, \quad g_{+}^{\prime}(x) \in B V\left(\mathbb{R}_{+} ; \mathbb{R}\right),
$$

and, for some small $\varepsilon>0$,

$$
\left\|g_{+}^{\prime}(x)-g_{*}^{\prime}(x)\right\|_{B V\left(\mathbb{R}_{+}\right)} \leq \varepsilon,
$$

where

$$
g_{+}^{\prime}(x)=g^{\prime}(x+)=\lim _{\hat{x} \rightarrow x+} \frac{g(\hat{x})-g(x)}{\hat{x}-x} ;
$$

$\left(\mathbf{H}_{\mathbf{2}}\right)$ The incoming flow $\bar{U}(y)=(\bar{u}, \bar{v}, \bar{p}, \bar{\rho}, \bar{Z})(y)$ at $x=0$ with bounded total variation is a small perturbation of the constant state $U_{\infty}=\left(u_{\infty}, 0, p_{\infty}, \rho_{\infty}, 0\right)$ and satisfies

$$
\bar{u}^{2}+\bar{v}^{2}>\bar{c}^{2}, \quad 0 \leq \bar{Z} \leq 1, \quad \bar{Z}(\infty)=0,
$$

where $u_{\infty}>c_{\infty}=\sqrt{\frac{\gamma p_{\infty}}{\rho_{\infty}}}$. In addition, there exists a positive constant $T_{*}$ such that

$$
\bar{T}(y)>T_{*}>0 .
$$

Assumption (1.8) in $\left(\mathbf{H}_{\mathbf{2}}\right)$ is to make sure that the reaction rate function $\phi(T)$ has a positive minimum value $L_{*}=\phi\left(T_{*}\right)$ which never vanishes. In a sense, this is a very realistic condition. Typically, $\phi(T)$ has the Arrhenius form as in [6]:

$$
\phi(T)=T^{\alpha} e^{-\frac{\mathcal{E}}{R T}},
$$

which vanishes only at absolutely zero temperature, where $\mathcal{E}$ is the action energy and $\alpha$ is a positive constant.

For the given bending wall $y=g(x)$, the domain and its boundary are defined as

$$
\Omega=\{(x, y): x>0, y>g(x)\}, \quad \Gamma=\{(x, y): x>0, y=g(x)\},
$$

and

$$
\mathbf{n}=\mathbf{n}(x, g(x))=\frac{\left(g^{\prime}(x),-1\right)}{\sqrt{1+\left(g^{\prime}(x)\right)^{2}}}
$$


is the outer normal vector to $\Gamma$ at point $x$ except the non-differential point. Regarding $x$ as a time-like variable, the planar flow problem can be formulated as the initial-boundary problem for system (1.1) with

\section{Cauchy condition:}

\section{Boundary condition:}

$$
\left.U\right|_{x=0}=\bar{U}(y)
$$

$$
\left.(u, v) \cdot \mathbf{n}\right|_{\Gamma}=0 .
$$

Definition 1.1 (Entropy solutions). A function $U \in B V_{l o c}(\Omega)$ is called an entropy solution to the initial-boundary value problem (1.1) and (1.10)-(1.11) in $\Omega \subset \mathbb{R}_{+}^{2}$ provided that, for any convex entropy pair $(\eta, q)$ with respect to $W=W(U)$ of (1.1), that is, $\nabla^{2} \eta(W) \geq 0$ and $\nabla q(W)=\nabla \eta(W) \nabla H(U(W))$, the following entropy inequality holds: For any $\psi \in$ $C_{0}^{\infty}\left(\mathbb{R}^{2}\right)$ with $\psi \geq 0$,

$$
\begin{aligned}
& \iint_{\Omega}\left(\eta(W(U)) \psi_{x}+q(W(U)) \psi_{y}+\nabla_{W} \eta(W(U)) G(U) \psi\right) d x d y \\
& +\int_{0}^{\infty} \eta(W(\bar{U}(y))) \psi(0, y) d y \geq 0 .
\end{aligned}
$$

The entropy inequality (1.12) directly implies that $U=U(x, y)$ is a weak solution of system (1.1) with (1.10)-(1.11):

$$
\iint_{\Omega}\left(W(U) \phi_{x}+H(U) \phi_{y}+G(U) \phi\right) d x d y+\int_{0}^{\infty} W(\bar{U}(y)) \phi(0, y) d y=0
$$

for any $\phi \in C_{0}^{\infty}\left(\mathbb{R}^{2}\right)$. This can be seen by choosing $\eta(W)= \pm W$.

Moreover, $\eta(W)=-\rho u S$ is an entropy which is convex with respect to $W$, while $q(W)=-\rho v S$ is the corresponding entropy flux, when $\rho>0$ and $u>c$, so that the entropy inequality (1.12) also implies the physical entropy condition (1.5).

For the non-reacting steady Euler system with supersonic state and certain physical boundaries, some analysis on the shocks and rarefaction waves has been made. For example, as described in [14, when a supersonic flow hits a sharp body or moves around a sharp corner, a supersonic shock is formed and attached to the body, or a rarefaction wave is generated by the corner. Such a physical phenomenon has been extensively studied for the Lipschitz boundaries or smooth boundaries that are small perturbations of a straight one. For instance, Zhang [45]-48] considered the two-dimensional steady supersonic potential flow past Lipschitz wedges or over bending walls and obtained the global existence and asymptotic behavior of entropy solutions in $B V$ which contain a strong shock with large vertex angle or a strong rarefaction wave. Later, similar results have been obtained for the full Euler equations for entropy solutions in $B V$ which contain a strong shock in ChenZhang-Zhu [8]. Moreover, based on [8], the stability and uniqueness of entropy solutions containing a strong shock by the wave-front tracking algorithm were established in ChenLi 5]. For the space dimension higher than two, the global existence of weak solutions for steady supersonic conical flow has been analyzed first in Lien-Liu [28] for isentropic Euler flow under the assumptions that the symmetrical cone has a small opening angle and the initial strength of the relatively strong shock is sufficiently weak, and has been studied then in Wang-Zhang [41] for steady potential flow past the cone with an arbitrary opening angle that is less than a critical value. On the other hand, the local/global existence for 
steady supersonic flow past cones or sharp corners with smooth boundaries has also been studied extensively in [15, 9, 10, 11, 40] and the references cited therein.

For the exothermically reacting Euler equations, the global existence of entropy solutions for the Cauchy problem was first established for the one-dimensional case that the reaction rate function is Lipschtiz and has a lower bound in Chen-Wagner [6] when the total variation of the initial data is bounded by a constant proportional to a parameter $\frac{1}{\gamma-1}$, by developing the Glimm-type fractional-step scheme. In Chen-Xiao-Zhang [7], the Cauchy problem for the two-dimensional steady case has first been studied, and the initialboundary value problem for supersonic reacting flow past a Lipschitz wedge with large or small angle has then been analyzed: When the total variation of both the initial data and the slope of the wedge boundary is suitably small, the global existence and asymptotic behavior of the entropy solutions have been established. For the multidimensional case, we also refer the reader to [13] for the details.

When the reaction rate function is discontinuous, we refer to [30, 36, 38, 39, 43, 44] and the references therein for the Riemann problem for the one-dimensional case. For further information on this topic and related combustion theories, we refer the reader to [17, 32, 34, 42.

In this paper, we establish the global existence and asymptotic behavior of entropy solutions for two-dimensional steady supersonic inviscid reacting flow over a Lipschitz bending wall. Our problem is different from [45] that has been done for the potential flow. The flow here is described by five equations with the reaction source terms, which may be viewed as the full Euler equations coupled with a nonhomogeneous transport equation owing to the reacting process. Thus the problem we consider can be formulated as an initial-boundary value problem for a hyperbolic system of balance laws, which involves a strong rarefaction wave. One of our main motivations is the mathematical difficulty that the heat released by the reaction may cause the total variation of the solution to increase along the flow direction, even in the one-dimensional case (cf. [3, 6, 16, 18, 22, 33]). One of our main results in this paper is to prove that the increase in the total variation of the solution is eventually bounded as a result of the uniform and exponential decay of the reactant along the flow direction.

To treat this nonlinear problem with the strong rarefaction wave and reaction source terms, one natural way is to combine the wave-front tracking algorithm with the fractionalstep technique, since it is more convenient to determine the position and control the strength of the strong rarefaction wave in the construction of approximate solutions. More precisely, to achieve this, we proceed in the following two steps:

We first study the homogeneous system by approximating the Lipschitz boundary with polylines and employing the ideas developed in Amadori [1] and Bressan [4] to construct approximate solutions $U^{\nu, h}$ of the initial-boundary value problem in each interval $((k-$ 1) $h, k h), k \in \mathbb{N}_{+}$. In this construction, our key observation is that the discontinuities of the 5th component $Z(x, y)$ propagate only along the 4 -contact discontinuities and is unchanged when they cross the other families of waves, especially for the non-physical waves. In addition, in dealing with the change in the strength of weak waves after they interact with strong rarefaction fronts, we impose the weights $W\left(\alpha_{i}, x,-\right), 1 \leq i \leq 4$, and $W(\epsilon, x,-)$ for weak waves and introduce a functional $F_{1}(U ; x)$ (see $\S 5$ below). 
The second step is to consider the reaction process that is related by (4.30)-(4.34) with the reaction step $h$. To do this, we employ the accurate Riemann solver to solve the Riemann problem with the Riemann data on the physical waves for the reaction step, and let the non-physical waves across line $x=k h$ directly when the reaction step occurs on it. We also remark that the orders of strong rarefaction fronts and the non-physical fronts are unchanged. However, in the proof of the convergence and consistency of the solutions, we first fix $h$ to take the limits for $\nu \rightarrow \infty$ and then let $h \rightarrow 0$. We do this in order to avoid the wave-fronts that may increase owing to the reaction process. Similar ideas have also been used in Amadori-Gosse-Guerra [2]. Finally, with the estimates on the wave interactions, we can first obtain the a priori bound on the total variation of the approximate solutions and then, by carrying out the steps as in [2, 19], we obtain the global existence of entropy solutions for problem (1.1) and (1.10)-(1.11).

For the asymptotic behavior of entropy solutions, we need further estimates on the approximate solutions $U^{\nu, h}$. The key step for the estimates required here is when the 1-generalized characteristics intersect with the boundary after they cross the rarefaction area. This is different from the wedge case that has been handled in [7]. Then, by applying the Glimm-Lax theory [20], one can derive the asymptotic behavior of entropy solutions.

Before concluding this section, we remark in passing that the global existence for the Cauchy problem of the one-dimensional hyperbolic systems of conservation laws with the initial data containing strong rarefaction waves has been considered by Lewicka in [24, 25]. The problem we are considering here is different from [24, 25], since our problem is of initial-boundary value type for the hyperbolic systems of balance laws including the reaction source terms. For the existence and $L^{1}$-stability for the Cauchy problem containing weak elementary waves (i.e., shocks, rarefaction waves, and contact discontinuities) for one-dimensional hyperbolic systems, we refer the reader to [23, 35] and the references therein. Also see Lewicka-Trivisa [26] for the $L^{1}$ well-posedness of the one-dimensional hyperbolic systems of conservation laws near solutions containing two large shocks.

The rest of this paper is organized as follows: In $\S 2$, we present the nonlinear waves and the Riemann solutions for the homogeneous equation (1.6) in the supersonic region. In $\S 3$, we consider the background solution for the homogeneous system as supersonic Euler flow (i.e., $Z=0$ ) past the convex Lipschitz bending wall $y=g_{*}(x)$. In $\S 4$, the approximate solutions for the initial-boundary value problem (1.1) and (1.10)-(1.11) are constructed by developing a fractional-step wave-front tracking algorithm. In $\S 5$, some weights are introduced to construct the Glimm-type functional. In $\S 6$, we consider various types of wave interactions between weak waves and strong rarefaction wave-fronts. Then we establish the uniform bound on the total variation of the approximate solutions for the homogeneous system (i.e., (2.1)). In $\S 7$, we study the $B V$-stability of the approximate solutions for the reacting step and establish the uniform bound on the total variation of the approximate solutions. In $\S 8$, the convergence and consistency of the approximate solutions are established and then the existence theorem is stated. In $\S 9$, we study the asymptotic behavior of entropy solutions. Finally, we give a detail proof of Lemma 2.1] in $\S 10$.

We also remark that, throughout this paper, $O(1)$ stands for the bounded quantities that depend only on the system. 


\section{Riemann Problem}

In this section, we present some basic properties of the homogeneous system of (1.1).

2.1. Homogeneous system. In the case when $G(U)$ is identically zero, system (1.6) becomes

$$
W(U)_{x}+H(U)_{y}=0 .
$$

With $x$ as the time-like variable, system (2.1) is hyperbolic for $u>c$. This system has five eigenvalues:

$$
\begin{aligned}
& \lambda_{j}=\frac{u v+(-1)^{\frac{j+3}{4}} c \sqrt{u^{2}+v^{2}-c^{2}}}{u^{2}-c^{2}}, \quad j=1,5, \\
& \lambda_{i}=\frac{v}{u}, \quad i=2,3,4
\end{aligned}
$$

and corresponding five linearly independent eigenvectors:

$$
\begin{aligned}
& \tilde{\mathbf{r}}_{j}=\left(-\lambda_{j}, 1, \rho\left(\lambda_{j} u-v\right), \frac{\rho\left(\lambda_{j} u-v\right)}{c^{2}}, 0\right)^{\top}, \quad j=1,5, \\
& \tilde{\mathbf{r}}_{2}=(u, v, 0,0,0)^{\top}, \quad \tilde{\mathbf{r}}_{3}=(0,0,0,1,0)^{\top}, \quad \tilde{\mathbf{r}}_{4}=(0,0,0,0,1)^{\top} .
\end{aligned}
$$

Let

$$
q=\sqrt{u^{2}+v^{2}}, \quad \theta=\arctan \left(\frac{v}{u}\right), \quad \theta_{\mathrm{ma}}=\arctan \left(\frac{c}{\sqrt{q^{2}-c^{2}}}\right),
$$

where $\theta_{\mathrm{ma}}$ is the Mach angle. Then

$$
\lambda_{j}=\tan \left(\theta+(-1)^{\frac{j+3}{4}} \theta_{\mathrm{ma}}\right), \quad j=1,5 ; \quad \lambda_{i}=\tan \theta, \quad i=2,3,4 .
$$

With the state variables $(q, \theta, p, \rho, Z)^{\top}$, we can rewrite the eigenvectors as

$$
\begin{aligned}
& \tilde{\mathbf{r}}_{1}=\left(-\tan \left(\theta-\theta_{\mathrm{ma}}\right), 1,-\rho q \sec \left(\theta-\theta_{\mathrm{ma}}\right) \sin \left(\theta_{\mathrm{ma}}\right),-\frac{\rho q}{c^{2}} \sec \left(\theta-\theta_{\mathrm{ma}}\right) \sin \left(\theta_{\mathrm{ma}}\right), 0\right)^{\top}, \\
& \tilde{\mathbf{r}}_{5}=\left(-\tan \left(\theta+\theta_{\mathrm{ma}}\right), 1, \rho q \sec \left(\theta+\theta_{\mathrm{ma}}\right) \sin \left(\theta_{\mathrm{ma}}\right), \frac{\rho q}{c^{2}} \sec \left(\theta+\theta_{\mathrm{ma}}\right) \sin \left(\theta_{\mathrm{ma}}\right), 0\right)^{\top}, \\
& \tilde{\mathbf{r}}_{2}=(\cos \theta, \sin \theta, 0,0,0)^{\top}, \quad \tilde{\mathbf{r}}_{3}=(0,0,0,1,0)^{\top}, \quad \tilde{\mathbf{r}}_{4}=(0,0,0,0,1)^{\top} .
\end{aligned}
$$

Moreover, we have the following properties whose proofs are given in $\S 10$.

Lemma 2.1. For $u>c$,

$$
\nabla_{U} \lambda_{j} \cdot \tilde{\mathbf{r}}_{j}>0, \quad j=1,5 ; \quad \nabla_{U} \lambda_{i} \cdot \tilde{\mathbf{r}}_{i}=0, \quad i=2,3,4 .
$$

Lemma 2.1 implies that the $j$-th characteristic fields are genuinely nonlinear for $j=1,5$, while the $i$-th characteristic fields, $i=2,3,4$, are linearly degenerate. Therefore, we can choose $\mathbf{r}_{j}=\kappa_{j} \tilde{r}_{j}$ with $\kappa_{j}=\frac{1}{\nabla_{U} \lambda_{j} \cdot \tilde{\mathbf{r}}_{j}}, j=1,5$, and $\mathbf{r}_{i}=\tilde{\mathbf{r}}_{i}, i=2,3,4$, such that

$$
\nabla_{U} \lambda_{j} \cdot \mathbf{r}_{j}=1, \quad j=1,5 \text {. }
$$


2.2. Nonlinear waves for system (2.1). In this subsection, we discuss nonlinear wave curves in the phase-space for system (2.1). As indicated in $\S 2.1$, system (2.1) admits five elementary waves that belong to the corresponding characteristic families for $u>c$.

The Rankine-Hugoniot conditions for system (2.1) with the discontinuity speed $s$ are

$$
s[W(U)]=[H(U)],
$$

where $[W(U)]=W\left(U_{+}\right)-W\left(U_{-}\right)$, and $U_{+}$and $U_{-}$are the above and below states of the discontinuity, respectively.

The $i$-contact wave curves $C_{i}\left(U_{0}\right), i=2,3,4$, through $U_{0}$ are

$$
C_{i}\left(U_{0}\right): p=p_{0}, s_{i}=\frac{v}{u}=\frac{v_{0}}{u_{0}} .
$$

That is, we can parameterize $C_{i}\left(U_{0}\right)$ as

$$
\begin{aligned}
& C_{2}\left(U_{0}\right): u=u_{0} e^{\sigma_{2}}, v=v_{0} e^{\sigma_{2}}, p=p_{0}, \rho=\rho_{0}, Z=Z_{0}, \\
& C_{3}\left(U_{0}\right): u=u_{0}, v=v_{0}, p=p_{0}, \rho=\rho_{0}+\sigma_{3}, Z=Z_{0}, \\
& C_{4}\left(U_{0}\right): u=u_{0}, v=v_{0}, p=p_{0}, \rho=\rho_{0}, Z=Z_{0}+\sigma_{4},
\end{aligned}
$$

where $C_{2}\left(U_{0}\right)$ corresponds the compressible vortex sheet, $C_{3}\left(U_{0}\right)$ the entropy wave, and $C_{4}\left(U_{0}\right)$ the reactant wave.

The $j$-th shock wave curves $S_{j}\left(U_{0}\right), j=1,5$, through $U_{0}$ are

$$
S_{j}^{-}\left(U_{0}\right):[p]=\frac{c_{0}^{2}}{\tilde{b}}[\rho],[u]=-s_{j}[v],[p]=\rho_{0}\left(s_{j} u_{0}-v_{0}\right)[v],[Z]=0, \quad \rho>\rho_{0},
$$

where $\tilde{b}=\frac{\gamma+1}{2}-\frac{\gamma-1}{2} \frac{\rho}{\rho_{0}}, \tilde{c}^{2}=\frac{c_{0}^{2}}{\tilde{b}} \frac{\rho}{\rho_{0}}$, and

$$
s_{j}=\frac{u_{0} v_{0}+(-1)^{\frac{j+3}{4}} \tilde{c} \sqrt{u_{0}^{2}+v_{0}^{2}-\tilde{c}^{2}}}{u_{0}^{2}-\tilde{c}^{2}}, \quad j=1,5 .
$$

The rarefaction wave curves $R_{j}\left(U_{0}\right), j=1,5$, in the phase-space through $U_{0}$ are given by

$$
R_{j}^{+}\left(U_{0}\right): d p=c^{2} d \rho, d u=-\lambda_{j} d v, \rho\left(\lambda_{j} u-v\right) d v=d p, d Z=0, \quad \rho<\rho_{0} .
$$

In the physical states $(q, \theta, p, \rho, Z)^{\top}, R_{j}\left(U_{0}\right), j=1,5$, can also be expressed as

$$
\begin{aligned}
& R_{1}^{+}\left(U_{0}\right): J(q, \mathcal{B})-\theta=J\left(q_{0}, \mathcal{B}_{0}\right)-\theta_{0}, \frac{q^{2}}{2}+\frac{c^{2}}{\gamma-1}=\frac{q_{0}^{2}}{2}+\frac{c_{0}^{2}}{\gamma-1}, \frac{p}{\rho^{\gamma}}=\frac{p_{0}}{\rho_{0}^{\gamma}}, Z=Z_{0}, \\
& R_{5}^{+}\left(U_{0}\right): J(q, \mathcal{B})+\theta=J\left(q_{0}, \mathcal{B}_{0}\right)+\theta_{0}, \frac{q^{2}}{2}+\frac{c^{2}}{\gamma-1}=\frac{q_{0}^{2}}{2}+\frac{c_{0}^{2}}{\gamma-1}, \frac{p}{\rho^{\gamma}}=\frac{p_{0}}{\rho_{0}^{\gamma}}, Z=Z_{0},
\end{aligned}
$$

where $U=(u, v, p, \rho, Z)^{\top}, U_{0}=\left(u_{0}, v_{0}, p_{0}, \rho_{0}, Z_{0}\right)^{\top}, q_{0}=\sqrt{u_{0}^{2}+v_{0}^{2}}, \theta_{0}=\arctan \left(\frac{v_{0}}{u_{0}}\right)$, and

$$
J(q, \mathcal{B}):=\int^{q} \frac{\sqrt{(\gamma+1) \mu^{2}-2(\gamma-1) \mathcal{B}}}{(\gamma-1) \mu \sqrt{2 \mathcal{B}-\mu^{2}}} d \mu,
$$


with

$$
\mathcal{B}:=\frac{q^{2}}{2}+\frac{c^{2}}{\gamma-1}, \quad \mathcal{B}_{0}:=\frac{q_{0}^{2}}{2}+\frac{c_{0}^{2}}{\gamma-1} .
$$

Note that the shock wave curve $S_{j}^{-}\left(U_{0}\right)$ contacts with $R_{j}^{+}\left(U_{0}\right)$ at $U_{0}$ up to second order for each $j=1,5$.

2.3. Riemann problem for the homogeneous system (2.1). For $\delta_{0}>0$, define

$$
D_{\delta_{0}}\left(U_{\infty}\right)=\left\{\begin{array}{c}
\left|J\left(q, \frac{q^{2}}{2}+\frac{c^{2}}{\gamma-1}\right)+\theta-J\left(q_{\infty}, \frac{q_{\infty}^{2}}{2}+\frac{c_{\infty}^{2}}{\gamma-1}\right)\right|<\delta_{0} \\
U: \quad\left|\frac{q^{2}}{2}+\frac{c^{2}}{\gamma-1}-\frac{q_{\infty}^{2}}{2}-\frac{c_{\infty}^{2}}{\gamma-1}\right|<\delta_{0}, \quad\left|\frac{p}{\rho^{\gamma}}-\frac{p_{\infty}}{\rho_{\infty}^{\gamma}}\right|<\delta_{0} \\
0 \leq Z<\delta_{0}, \quad \delta_{0}+\theta_{\text {crit }}<\theta<\delta_{0}
\end{array}\right\},
$$

where

$$
\theta_{\text {crit }}:=\inf _{\theta}\left\{\theta: U \in R_{5}^{+}\left(U_{\infty}\right), u>c_{*}, q<q_{*}\right\}
$$

with the critical sonic speed $c_{*}$ and critical speed $q_{*}$ given, respectively, by

$$
c_{*}=\sqrt{\frac{(\gamma-1) u_{\infty}^{2}}{\gamma+1}+\frac{2 c_{\infty}^{2}}{\gamma+1}}, \quad q_{*}=\sqrt{u_{\infty}^{2}+\frac{2 c_{\infty}^{2}}{\gamma-1}}
$$

which depend only on the unperturbed initial state.

In what follows, $\delta_{0}$ is always chosen small enough so that $\overline{D_{\delta_{0}}\left(U_{0}\right)} \subset\{U: u>c\}$.

We now consider the Riemann problem for (2.1) in $D_{\delta_{0}}\left(U_{\infty}\right)$ with

$$
\left.U\right|_{x=\hat{x}}= \begin{cases}U_{a}, & y>\hat{y}, \\ U_{b}, & y<\hat{y},\end{cases}
$$

where the constant states $U_{a}$ and $U_{b}$ denote the above state and below state with respect to line $y=\hat{y}$, respectively.

When $\left|U_{a}-U_{b}\right|$ is sufficiently small, following Lax [21], we can parameterize any physically admissible wave curve in a neighborhood of $D_{\delta_{0}}\left(U_{\infty}\right)$ by

$$
\alpha_{j} \mapsto \Phi_{j}\left(\alpha_{j}, U_{b}\right)
$$

with $\Phi_{j} \in C^{2}\left(\left(-\delta_{0}, \delta_{0}\right) \times D_{\delta_{0}}\left(U_{\infty}\right)\right)$ and

$$
\left.\Phi_{j}\right|_{\alpha_{j}=0}=U_{b},\left.\quad \frac{\partial \Phi_{j}}{\partial \alpha_{j}}\right|_{\alpha_{j}=0}=\mathbf{r}_{j}\left(U_{b}\right) .
$$

Then $\alpha_{j}>0$ along $R_{j}^{+}\left(U_{b}\right)$, while $\alpha_{j}<0$ for $S_{j}^{-}\left(U_{b}\right)$ with $1 \leq j \leq 5$.

For simplicity, let

$$
\Phi\left(\alpha_{5}, \alpha_{4}, \alpha_{3}, \alpha_{2}, \alpha_{1} ; U_{b}\right):=\Phi_{5}\left(\alpha_{5}, \Phi_{4}\left(\alpha_{4}, \Phi_{3}\left(\alpha_{3}, \Phi_{2}\left(\alpha_{2}, \Phi_{1}\left(\alpha_{1}, U_{b}\right)\right)\right)\right)\right) .
$$

Then we have

Lemma 2.2. There exists $0<\delta_{1} \ll 1$ such that, for any states $U_{a}, U_{b} \in D_{\delta_{0}}\left(U_{\infty}\right)$ with $\left|U_{a}-U_{b}\right|<\delta_{1}$, problem (2.1) and (2.20) admits a unique admissible solution consisting of five elementary waves. In addition, state $U_{a}$ can be solved by

$$
U_{a}=\Phi\left(\alpha_{5}, \alpha_{4}, \alpha_{3}, \alpha_{2}, \alpha_{1} ; U_{b}\right),
$$


with $\left.\Phi\right|_{\alpha_{1}=\alpha_{2}=\alpha_{3}=\alpha_{4}=\alpha_{5}=0}=U_{b}$ and $\left.\frac{\partial \Phi}{\partial \alpha_{j}}\right|_{\alpha_{1}=\alpha_{2}=\alpha_{3}=\alpha_{4}=\alpha_{5}=0}=\mathbf{r}_{j}\left(U_{b}\right), 1 \leq j \leq 5$.

Remark 2.1. For any state $U_{b} \in D_{\delta_{0}}\left(U_{\infty}\right)$, we can also parameterize the whole curve $R_{5}\left(U_{b}\right)$ by solving the equation:

$$
\frac{d U_{\mathrm{Ra}}\left(\sigma, U_{b}\right)}{d \sigma}=\mathbf{r}_{5}\left(U_{\mathrm{Ra}}\left(\sigma, U_{b}\right)\right)
$$

with $\left.\Phi\right|_{\sigma=0}=U_{b}$. Then $\Phi_{5}$ can be extended by $\Phi_{5}\left(\sigma, U_{b}\right)=U_{\operatorname{Ra}}\left(\sigma, U_{b}\right)$ for $\sigma \geq 0$.

\section{Strong Rarefaction Waves for Euler Flow}

In this section, we investigate the background solution for the Euler equations for steady supersonic Euler flow with $Z=0$ for (2.1) over a convex Lipschitz wall $g_{*}(x)$ and the constant incoming flow $U_{\infty}=\left(u_{\infty}, 0, p_{\infty}, \rho_{\infty}, 0\right)^{\top}$. We call system (2.1) with $Z=0$ simply as the Euler equations. We first consider a special case $g_{*}(x)=g_{* \Delta}(x)$ with

$$
g_{* \Delta}(x)= \begin{cases}0, & x \leq 0, \\ g_{*}\left(x_{k}^{*}\right)+\left(x-x_{k}^{*}\right) \tan \theta_{k}^{*}, & x \in\left(x_{k}^{*}, x_{k+1}^{*}\right), k \geq 0,\end{cases}
$$

where $\left\{x_{k}^{*}\right\}_{k=0}^{\infty}$ is a sequence of points with $x_{0}^{*}=0,0<x_{k}^{*}<x_{k+1}^{*}, k \geq 1, \Delta x^{*}=x_{k+1}^{*}-x_{k}^{*}$, and

$$
\theta_{k}^{*}=\arctan \left(\frac{g_{*}\left(x_{k+1}^{*}\right)-g_{*}\left(x_{k}^{*}\right)}{\Delta x^{*}}\right)
$$

satisfies $\theta_{\text {crit }}<\theta_{k+1}^{*}<\theta_{k}^{*}<0$ for $k \geq 0$.

When the Euler flow passes the convex Lipschitz wall $g_{* \Delta}(x)$, some rarefaction waves are generated by the corner points of the wall.

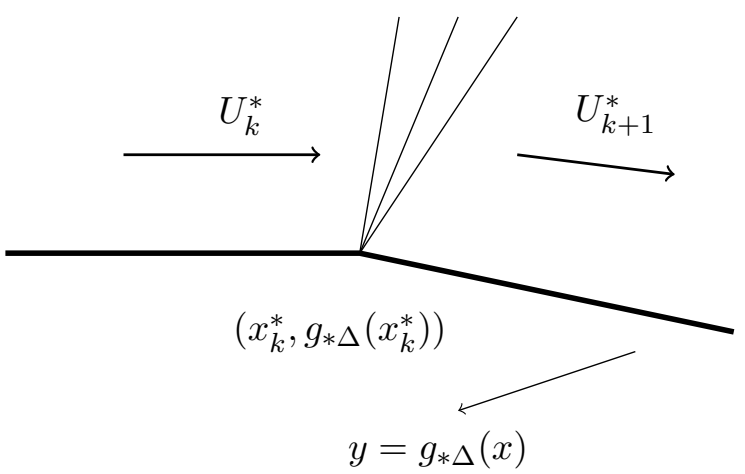

Fig. 3.1. Steady supersonic Euler flow past a piecewise convex wall

Then we can employ the methods that have been given in [14, 40] to construct the solution. We summary this as the following theorem.

Theorem 3.1. Suppose that $g_{*}(x) \equiv g_{* \Delta}(x)$ and $\theta_{k}^{*} \in\left(\theta_{\text {crit }}, 0\right), k \geq 0$. Let $U_{k}^{*}=$ $\left(u_{k}^{*}, v_{k}^{*}, p_{k}^{*}, \rho_{k}^{*}, 0\right)$ be the incoming states that depend on the initial data $U_{\infty}$. Then there exists a unique solution $U_{\Delta}^{*}(x, y)$ for the Euler equations (2.1) over the convex wall $g_{* \Delta}(x)$ in the supersonic region $\left\{U: u>c_{*}, q<q_{*}\right\}$ which consists of strong rarefaction waves that are generated by the corner points $\left(x_{k}^{*}, g_{* \Delta}\left(x_{k}^{*}\right)\right), k \geq 0$, where $\theta_{\text {crit }}, c_{*}$, and $q_{*}$ are defined as in (2.18)-(2.19). 


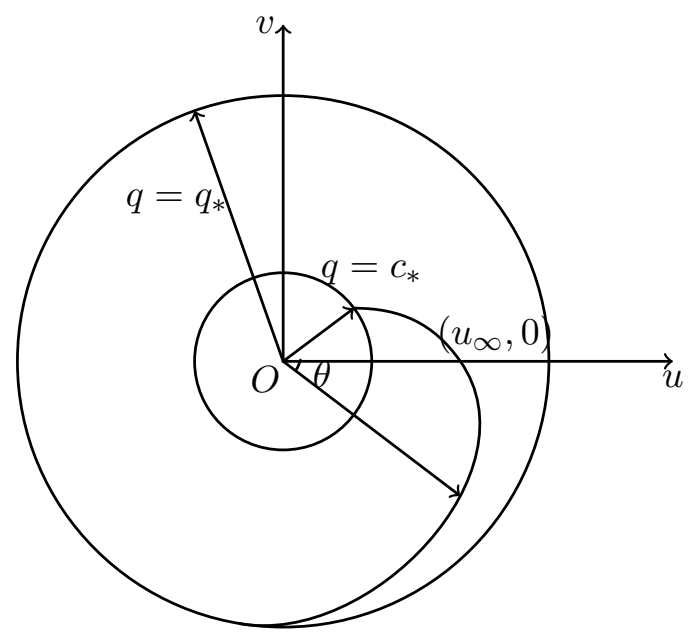

Fig. 3.2. The epicycloid issues from $\left(u_{\infty}, 0\right)$ in the $(u, v)$-plane

As a corollary, we can prove a similar result for the general case of Theorem 3.1 ,

Corollary 3.1. Suppose that $\arctan \left(g_{*}^{\prime}(x)\right) \in\left(\theta_{\text {crit }}, 0\right)$, and $g_{*}^{\prime}(\cdot)$ is monotone decreasing. If $T V .\left(g_{*}^{\prime}(\cdot)\right)<\infty$, then there exists a unique solution $U^{*}(x, y)$ in the supersonic region $\left\{U: u>c_{*}, q<q_{*}\right\}$ which connects $U_{\infty}$ by a strong rarefaction wave $R_{5}^{+}\left(U_{\infty}\right)$ for the Euler equations (2.1) over the convex wall $y=g_{*}(x)$, where $\theta_{\text {crit }}, c_{*}$, and $q_{*}$ are defined as in (2.18) -(2.19).

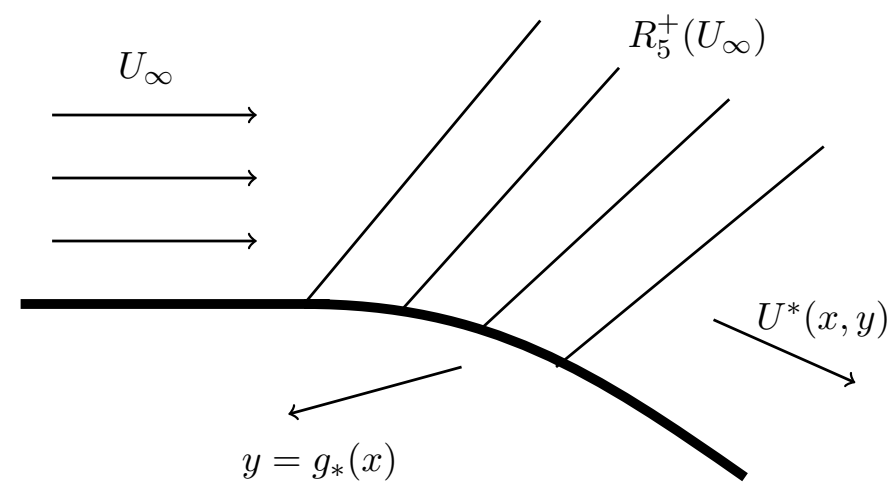

Fig. 3.3. Supersonic Euler flow past a convex bending wall

\section{Construction of Approximate Solutions}

In this section, we construct a family of global approximate solutions to system (1.1) by employing a fractional-step scheme based on the wave-front tracking algorithm. As a first step, we need to approximate domain $\Omega$ by $\Omega_{h}$ defined below.

Let $h=\Delta x>0$ be the mesh length in the $x$-direction. Then we choose a set of points $\left\{A_{k}\right\}_{k=0}$ with $A_{k}=\left(x_{k}, g_{k}\right):=(k h, g(k h))$ on the boundary that connects $A_{k}$ to $A_{k+1}$ in 
order. Denote

$$
\begin{aligned}
& \theta_{0}=\arctan g_{0}, \quad \theta_{k}=\arctan \left(\frac{g_{k+1}-g_{k}}{h}\right), k \geq 1, \\
& \omega_{0}=\arctan \left(\frac{g\left(x_{0}\right)-g(0)}{x_{0}}\right), \quad \omega_{k}=\theta_{k}-\theta_{k-1}, k \geq 1,
\end{aligned}
$$

where $\omega_{k}$ represents the change of angle $\theta_{k-1}$ at the turning points $A_{k}, k \geq 1$. Define

$$
\begin{aligned}
& g_{h}(x)=g_{k}+(x-k h) \tan \theta_{k} \quad \text { for any } x \in[k h,(k+1) h), k \geq 0, \\
& \Omega_{h, k}=\left\{(x, y): k h \leq x<(k+1) h, y>g_{h}(x)\right\}, \\
& \Gamma_{h, k}=\left\{(x, y): k h \leq x<(k+1) h, y=g_{h}(x)\right\}, \\
& \Omega_{h}=\bigcup_{k \geq 0} \Omega_{h, k}, \quad \Gamma_{h}=\bigcup_{k \geq 0} \Gamma_{h, k} .
\end{aligned}
$$

Let $\mathbf{n}_{k}$ be the outer unit normal vector to $\Gamma_{h, k}$ :

$$
\mathbf{n}_{k}=\frac{\left(g_{k+1}-g_{k},-x_{k+1}+x_{k}\right)}{\sqrt{\left(g_{k+1}-g_{k}\right)^{2}+\left(x_{k+1}-x_{k}\right)^{2}}}=\left(\sin \theta_{k},-\cos \theta_{k}\right) .
$$

The approximate solutions for system (2.1) are constructed by an induction procedure, together with the fractional-step wave-front tracking scheme. That is, if the solution has been constructed for $x \leq(k-1) h$, then, for fixed $h$, we solve the homogeneous system (2.1) between $(k-1) h<x<k h$. Finally, we solve the nonhomogeneous problem for system (2.1) from $x=k h-$ to $x=k h+$ with initial data taking at $x=k h-$. The details of the construction can be seen in $\S 4.1$ below.

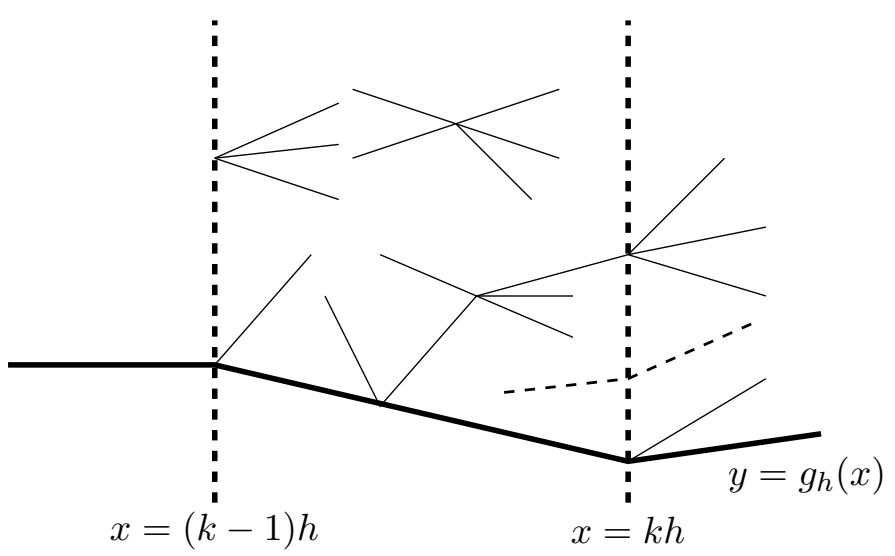

Fig. 4.1. Wave-front algorithm with the reacting steps

4.1. Riemann solvers for the homogeneous system (2.1). As mentioned in $\S 2$, the Riemann problem:

$$
W(U)_{x}+H(U)_{y}=0,\left.\quad U\right|_{x=\hat{x}}= \begin{cases}U_{a}, & y>\hat{y} \\ U_{b}, & y<\hat{y}\end{cases}
$$


admits a unique self-similar solution given by at most four states separated by shocks, contact discontinuities, or rarefaction waves. More precisely, the solution is inductively defined by

$$
U_{0}=U_{b}, \quad U_{i}=\Phi_{i}\left(\alpha_{i}, U_{i-1}\right), 1 \leq i \leq 5, \quad U_{5}=U_{a} .
$$

Following [1, 4], there are two procedures to define the approximate solutions to the Riemann problem (4.1): The accurate Riemann solver and the simplified Riemann solver.

Accurate Riemann solver. For any $\nu \in \mathbb{N}$, a $\nu$-approximate solution $U^{\nu}$ to the Riemann problem at any jump point $(\hat{x}, \hat{y})$ is defined by dividing every rarefaction wave into $\nu$ parts. That is, if $\alpha_{1}>0$, then set $U_{0,0}=U_{b}, U_{0, \nu}=U_{b}$, and

$$
U_{0, k}=\Phi_{1}\left(\frac{k}{\nu} \alpha_{1}, U_{0, k-1}\right), \quad y_{1, k}=\hat{y}+(x-\hat{x}) \lambda_{1}\left(U_{0, k}\right) \quad \text { for } 1 \leq k \leq \nu .
$$

In place of the 1-rarefaction wave, define

$$
U_{A}^{\nu}\left(U_{b}, U_{a}\right)= \begin{cases}U_{l}, & y<y_{1,1}, \\ U_{0, k}, & y_{1, k}<y<y_{1, k+1}, \\ U_{1}, & y_{1, \nu}<y<\hat{y}+(x-\hat{x}) \lambda_{1}^{*}\end{cases}
$$

for some $\lambda_{1}^{*} \in\left(\max \lambda_{1}, \min \lambda_{2(3,4)}\right)$. Then $U_{A}^{\nu}$ in $\left\{y: y<\hat{y}+(x-\hat{x}) \lambda_{1}^{*}\right\}$ given by (4.3) is called an approximate 1-rarefaction wave, which stands for the accurate $\nu$-Riemann solver. For simplicity, we still use $\alpha_{1}$ to denote both the wave given by (4.3) and its magnitude. The discontinuities in (4.3) are called 1-rarefaction fronts, and the magnitude of each front is $\frac{\alpha_{1}}{\nu}$.

In the same way, we can define the approximate 5-rarefaction wave $U_{A}^{\nu}$ with magnitude $\alpha_{5}$ in domain $\left\{y: y>\hat{y}+(x-\hat{x}) \lambda_{5}^{*}\right\}$ for some $\lambda_{5}^{*} \in\left(\max \lambda_{2(3,4)}, \min \lambda_{5}\right)$. It also contains $\nu$ 5-rarefaction fronts, and the magnitude of each front is $\frac{\alpha_{5}}{\nu}$.

In this construction, the $i$-shocks, $i=1,5$, and $j$-contact discontinuities, $j=2,3,4$, are not modified at all.

Simplified Riemann solver. As described in 1, 4, the simplified Riemann solver puts together all the new waves in a single non-physical front which travels faster than all the characteristic speeds. This is defined for the following two cases:

Case 1. Let $1 \leq i \leq j \leq 5$ be the families of the two incoming wave-fronts interacting at $(\hat{x}, \hat{y})$. Assume that the below, middle, and above states $\left\{U_{b}, U_{m}, U_{a}\right\}$ before interaction are connected by

$$
U_{m}=\Phi_{j}\left(\beta, U_{b}\right), \quad U_{a}=\Phi_{i}\left(\alpha, U_{m}\right) .
$$

Define the auxiliary above state:

$$
U_{a}^{\prime}= \begin{cases}\Phi_{j}\left(\beta, \Phi_{i}\left(\alpha, U_{b}\right)\right), & j>i, \\ \Phi_{j}\left(\alpha+\beta, U_{b}\right), & j=i .\end{cases}
$$

Choose a constant $\hat{\lambda}$ such that

$$
\hat{\lambda}>\sup _{i, U}\left\{\lambda_{i}(U): U \in D_{\delta_{0}}\left(U_{\infty}\right), 1 \leq i \leq 5\right\} .
$$


Then, in a forward neighbourhood of point $(\hat{x}, \hat{y})$, we define the approximate solution $U_{S}\left(U_{b}, U_{m}, U_{a}\right)$ to problem (4.1) as follows:

$$
U_{S}\left(U_{b}, U_{m}, U_{a}\right)= \begin{cases}U_{A}^{\nu}\left(U_{b}, U_{a}^{\prime}\right), & y-\hat{y}<\hat{\lambda}(x-\hat{x}), \\ U_{a}, & y-\hat{y}>\hat{\lambda}(x-\hat{x}),\end{cases}
$$

where $U_{A}^{\nu}\left(U_{b}, U_{a}^{\prime}\right)$ is the accurate Riemann solver constructed as in (4.3) which contains at most two wave-fronts. The part

$$
U_{\mathrm{np}}=\left\{\begin{array}{cc}
U_{a}^{\prime}, & y-\hat{y}<\hat{\lambda}(x-\hat{x}), \\
U_{a}, & y-\hat{y}>\hat{\lambda}(x-\hat{x})
\end{array}\right.
$$

is called a non-physical wave-front whose strength is defined to be $\left|U_{a}-U_{a}^{\prime}\right|$.

Case 2. A non-physical wave-front with strength $\epsilon$ hits a wave-front of the $i$-characteristic family at $(\hat{x}, \hat{y})$ from the below for some $1 \leq i \leq 5$.

Suppose that the below, middle, and above states $\left\{U_{b}, U_{m}, U_{a}\right\}$ before the interaction are connected by

$$
\left|U_{m}-U_{b}\right|=\epsilon, \quad U_{a}=\Phi_{i}\left(\alpha, U_{m}\right) .
$$

Then the approximate solution $U_{S}\left(U_{b}, U_{m}, U_{a}\right)$ to problem (4.1) is defined as follows:

$$
U_{S}\left(U_{b}, U_{m}, U_{a}\right)= \begin{cases}U_{b}, & y-\hat{y}<\lambda\left(U_{b}\right)(x-\hat{x}), \\ \Phi_{i}\left(\alpha, U_{b}\right), & \lambda\left(U_{b}\right)(x-\hat{x})<y-\hat{y}<\hat{\lambda}(x-\hat{x}), \\ U_{a}, & y-\hat{y}>\hat{\lambda}(x-\hat{x}) .\end{cases}
$$

4.2. Initial-boundary value problems for the homogeneous system (2.1). To study the flow past a corner point, we consider the following initial-boundary value problem:

$$
\begin{cases}W(U)_{x}+H(U)_{y}=0 & \text { in } \Omega_{h, k}, \\ \left.U\right|_{x=x_{k-1}}=U_{k-1}, & \\ (u, v) \cdot \mathbf{n}_{k}=0 & \text { on } \Gamma_{h, k},\end{cases}
$$

where $U_{k}$ is a constant state.

To describe the interaction and reflection on the boundary, we first need the following lemmas.

Lemma 4.1. Let $\left\{U_{1}, U_{2}\right\}$ be the two constant states related by

$$
U_{1}=\Phi_{5}\left(\alpha ; U_{2}\right) .
$$

Then, for $|\varepsilon| \ll 1$ sufficiently small, there exists a $C^{2}$-function $\Psi$ such that

$$
U_{2}=\Psi\left(\alpha ; U_{1}\right) .
$$

Moreover,

$$
\left.\frac{\partial \Psi}{\partial \alpha}\right|_{\alpha=0}=-\left.\frac{\partial \Phi_{5}}{\partial \alpha}\right|_{\alpha=0}=-\mathbf{r}_{5}\left(U_{1}\right)
$$


Proof. Since

$$
\nabla_{U_{2}} \Phi_{5}\left(0 ; U_{2}\right)=I
$$

then, by the implicit function theorem, for $|\alpha|$ sufficiently small, we can find a $C^{2}$-function $\Psi$ such that

$$
U_{2}=\Psi\left(\alpha ; U_{1}\right) \quad \text { for }|\alpha| \ll 1 .
$$

Therefore, (4.10) can be reduce to

$$
U_{1}=\Phi_{5}\left(\alpha ; \Psi\left(\alpha ; U_{1}\right)\right),
$$

which leads to

$$
\left.\frac{\partial \Psi}{\partial \alpha}\right|_{\alpha=0}=-\left.\frac{\partial \Phi}{\partial \alpha}\right|_{\alpha=0}=-\mathbf{r}_{5}\left(U_{1}\right) .
$$

This completes the proof.

Then we have

Lemma 4.2. Let $U_{k-1}=\left(u_{k-1}, v_{k-1}, p_{k-1}, \rho_{k-1}, Z_{k-1}\right)$ be the constant state near the boundary $\Gamma_{h, k-1}$ with $U_{k-1} \cdot\left(\boldsymbol{n}_{k-2}, 0,0,0\right)=0$.

(i) If $\left|\omega_{k-1}\right| \ll 1$, then there exists a unique solution $\left(\varepsilon_{5}, U_{k}\right)$ that solves the problem:

$$
\left\{\begin{array}{l}
\Phi_{5}\left(\varepsilon_{5} ; U_{k}\right)=U_{k-1}, \\
U_{k} \cdot\left(\boldsymbol{n}_{k-1}, 0,0,0\right)=0 .
\end{array}\right.
$$

Moreover, there exists $\tilde{K}_{\mathrm{b}}<0$ such that

$$
\varepsilon_{5}=\tilde{K}_{\mathrm{b}} \omega_{k-1},
$$

where the bound of $\tilde{K}_{\mathrm{b}}$ depends only on the system.

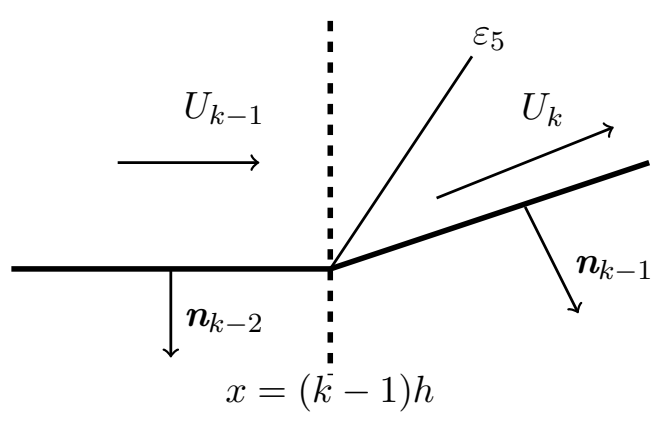

Fig. 4.2. Small waves generated by the corner points

(ii) If $\omega_{k-1} \in\left(\theta_{\text {crit }}+\theta_{k-1}, 0\right)$ with $\theta_{k-1}=\sum_{i=0}^{k-1}\left|\omega_{i}\right|$ and $\theta_{k-1}<-\theta_{\text {crit }}$, then there exists a unique solution $\left(\varepsilon_{5}, U_{k}\right)$ that consists of a strong rarefaction wave generated by 
the corner point $\left(x_{k-1}, g_{k-1}\right)$ and satisfies the equations:

$$
\begin{aligned}
& \theta\left(U_{\mathrm{Ra}}\left(-\varepsilon_{5}, U_{k-1}\right)\right)-\theta\left(U_{k-1}\right)=\omega_{k-1}, \\
& \left(J\left(q, \frac{q^{2}}{2}+\frac{c^{2}}{\gamma-1}\right)+\theta\right)\left(U_{\mathrm{Ra}}\left(-\varepsilon_{5}, U_{k-1}\right)\right)=\left(J\left(q, \frac{q^{2}}{2}+\frac{c^{2}}{\gamma-1}\right)+\theta\right)\left(U_{k-1}\right), \\
& \left(\frac{q^{2}}{2}+\frac{c^{2}}{\gamma-1}\right)\left(U_{\mathrm{Ra}}\left(-\varepsilon_{5}, U_{k-1}\right)\right)=\left(\frac{q^{2}}{2}+\frac{c^{2}}{\gamma-1}\right)\left(U_{k-1}\right), \\
& \left(\frac{p}{\rho^{\gamma}}\right)\left(U_{\mathrm{Ra}}\left(-\varepsilon_{5}, U_{k-1}\right)\right)=\left(\frac{p}{\rho^{\gamma}}\right)\left(U_{k-1}\right) \\
& Z_{k}=Z_{k-1},
\end{aligned}
$$

and

$$
U_{k}=U_{\mathrm{Ra}}\left(-\varepsilon_{5}, U_{k-1}\right),
$$

where $U_{\mathrm{Ra}}\left(-\varepsilon_{5}, U_{k-1}\right)$ is the parametrization of $R_{5}^{+}\left(U_{k-1}\right)$ given in Remark 2.1 . Moreover,

$$
\varepsilon_{5}=K_{\mathrm{b}}^{\prime} \omega_{k-1},
$$

where the bound of $K_{\mathrm{b}}^{\prime}$ depends only on the system.

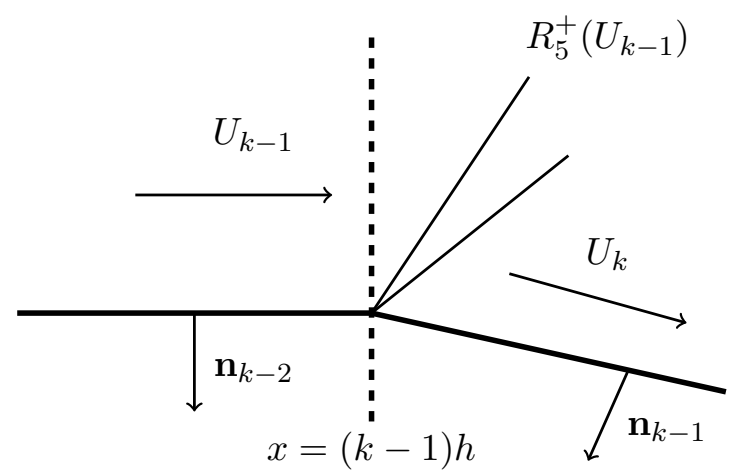

Fig. 4.3. Strong rarefaction waves generated by the corner points

Proof. We divide the proof into two steps.

1. By Lemma 4.1, there exists a $C^{2}$-function $\Psi$ such that

$$
\begin{aligned}
& U_{k}=\Psi\left(\varepsilon_{5} ; U_{k-1}\right) \quad \text { for }\left|\varepsilon_{5}\right| \ll 1, \\
& \left.\frac{\partial \Psi}{\partial \varepsilon_{5}}\right|_{\varepsilon_{5}=0}=-\left.\frac{\partial \Phi}{\partial \varepsilon_{5}}\right|_{\varepsilon_{5}=0}=-\mathbf{r}_{5}\left(U_{k-1}\right) .
\end{aligned}
$$

Then (4.13) can be reduced to

$$
\Psi\left(\varepsilon_{5} ; U_{k-1}\right) \cdot\left(\mathbf{n}_{k-1}, 0,0,0\right)=0 .
$$

Note that $U_{k-1} \cdot\left(\mathbf{n}_{k-2}, 0,0,0\right)=0$. Then

$$
\arctan \left(\frac{v_{k-1}}{u_{k-1}}\right)=\theta_{k-2}, \quad \lambda_{5}\left(U_{k-1}\right)=\tan \left(\theta_{k-2}+\theta_{\mathrm{ma}}\left(U_{k-1}\right)\right) .
$$


Therefore, using Lemma 2.1, we have

$$
\begin{aligned}
& \left.\frac{\partial}{\partial \varepsilon_{5}}\left(\Psi\left(\varepsilon_{5} ; U_{k-1}\right) \cdot\left(\mathbf{n}_{k-1}, 0,0,0\right)\right)\right|_{\varepsilon_{5}=\omega_{k-1}=0} \\
& =-\mathbf{r}_{5}\left(U_{k-1}\right) \cdot\left(\mathbf{n}_{k-2}, 0,0,0\right)=-\frac{\kappa_{5}\left(U_{k-1}\right) \cos \left(\theta_{\mathrm{ma}}\left(U_{k-1}\right)\right)}{\cos \left(\theta_{k-2}+\theta_{\mathrm{ma}}\left(U_{k-1}\right)\right)}<0 .
\end{aligned}
$$

Then, by the implicit function theorem, $\varepsilon_{5}$ can be solved as a $C^{2}$-function of $\left(\omega_{k-1}, U_{k-1}\right)$ with

$$
\varepsilon_{5}=\varepsilon_{5}\left(\omega_{k-1}, U_{k-1}\right)
$$

which leads to the existence of solution $\left(\varepsilon_{5}, U_{k}\right)$.

Finally, to establish estimate (4.14), we need to compute $\left.\frac{\partial \varepsilon_{5}}{\partial \omega_{k-1}}\right|_{\omega_{k-1}=0}$. To this end, differentiating (4.13) with respect to $\omega_{k-1}$ and letting $\omega_{k-1}=0$ yield

$$
-\left.\mathbf{r}_{5}\left(U_{k}\right) \cdot\left(\mathbf{n}_{k-1}, 0,0,0\right) \frac{\partial \varepsilon_{5}}{\partial \omega_{k-1}}\right|_{\omega_{k-1}=0}+U_{k} \cdot\left(\cos \theta_{k-2}, \sin \theta_{k-2}, 0,0,0\right)=0 .
$$

Therefore,

$$
\left.\frac{\partial \varepsilon_{5}}{\partial \omega_{k-1}}\right|_{\omega_{k-1}=0}=-\frac{\cos \left(\theta_{k-2}+\theta_{\mathrm{ma}}\left(U_{k-1}\right)\right)}{\kappa_{5}\left(U_{k-1}\right) q\left(U_{k-1}\right) \cos \left(\theta_{\mathrm{ma}}\left(U_{k-1}\right)\right)}<0 .
$$

2. Since

$$
\begin{aligned}
& \left.\nabla_{U} \theta(U)\right|_{U=U_{\mathrm{Ra}}\left(-\varepsilon_{5}, U_{k-1}\right)}=\left(-\frac{\sin \left(\theta\left(U_{\mathrm{Ra}}\left(-\varepsilon_{5}, U_{k-1}\right)\right)\right)}{q\left(U_{\mathrm{Ra}}\left(-\varepsilon_{5}, U_{k-1}\right)\right)}, \frac{\cos \left(\theta\left(U_{\mathrm{Ra}}\left(-\varepsilon_{5}, U_{k-1}\right)\right)\right)}{q\left(U_{\mathrm{Ra}}\left(-\varepsilon_{5}, U_{k-1}\right)\right)}, 0,0,0\right), \\
& \left.\frac{\partial U}{\partial \varepsilon_{5}}\right|_{U=U_{\mathrm{Ra}}\left(-\varepsilon_{5}, U_{k-1}\right)}=-\mathbf{r}_{5}\left(U_{\mathrm{Ra}}\left(-\varepsilon_{5}, U_{k-1}\right)\right) .
\end{aligned}
$$

Then

$$
\begin{aligned}
\left.\frac{\partial \theta(U)}{\partial \varepsilon_{5}}\right|_{U=U_{\mathrm{Ra}}\left(-\varepsilon_{5}, U_{k-1}\right)} & =\left.\left(\nabla_{U} \theta(U) \cdot \frac{\partial U}{\partial \varepsilon_{5}}\right)\right|_{U=U_{\mathrm{Ra}}\left(-\varepsilon_{5}, U_{k-1}\right)} \\
& =-\left.\frac{\kappa_{5}(U) \cos \theta_{\mathrm{ma}}(U)}{q(U) \cos \left(\theta(U)+\theta_{\mathrm{ma}}(U)\right)}\right|_{U=U_{\mathrm{Ra}}\left(-\varepsilon_{5}, U_{k-1}\right)}<0
\end{aligned}
$$

for any $U_{k} \in D_{\delta_{0}}\left(U_{\infty}\right)$, which implies that (4.15) has a unique solution $\varepsilon_{5}$ with

$$
\varepsilon_{5}=\varepsilon_{5}\left(\omega_{k-1}, U_{k-1}\right)
$$

Moreover, we have

$$
\varepsilon_{5}=\left(\left.\int_{0}^{1} \frac{\partial \theta(U)}{\partial \varepsilon_{5}}\right|_{U=U_{\mathrm{Ra}}\left(-\sigma, U_{k-1}\right)} d \sigma\right)^{-1} \omega_{k} .
$$

Hence, from equations (4.16)-(4.19), we can obtain the solution to problem (4.13) for $\omega_{k}<0$. This completes the proof. 
Next, we consider some waves that interact the boundary and then reflect. Assume that a 1 -wave $\alpha_{1}$ hits $\Gamma_{h, k-1}$ at the non-corner point $A(\hat{x}, \hat{y})$ with $(k-1) h<\hat{x}<k h$ for some $k>0$, and let

$$
U_{a}=\Phi_{1}\left(\alpha_{1} ; U_{b}\right), \quad U_{b} \cdot\left(\mathbf{n}_{k-1}, 0,0,0\right)=0 .
$$

We consider the problem:

$$
\begin{cases}W(U)_{x}+H(U)_{y}=0 & \text { for } x>\hat{x}, y>\hat{y}, \\ \left.U\right|_{x=\hat{x}}=U_{a}, & \\ (u, v) \cdot \mathbf{n}_{k-1}=0 & \text { on } \Gamma_{h, k-1} .\end{cases}
$$

Lemma 4.3. Let $\left\{U_{b}, U_{a}\right\}$ be the constant states given as above and satisfy (4.23). Then problem (4.24) admits a unique solution $\left(\varepsilon_{5}, U_{a}^{\prime}\right)$ that solves the following problem:

$$
\left\{\begin{array}{l}
\Phi_{5}\left(\varepsilon_{5} ; U_{a}^{\prime}\right)=U_{a}, \\
U_{a}^{\prime} \cdot\left(\boldsymbol{n}_{k-1}, 0,0,0\right)=0 .
\end{array}\right.
$$

Moreover,

$$
\varepsilon_{5}=K_{b 1} \alpha_{1},
$$

with $\left.K_{b 1}\right|_{\alpha_{1}=0}=1$, and the bound of $K_{b 1}$ depends only on the system.

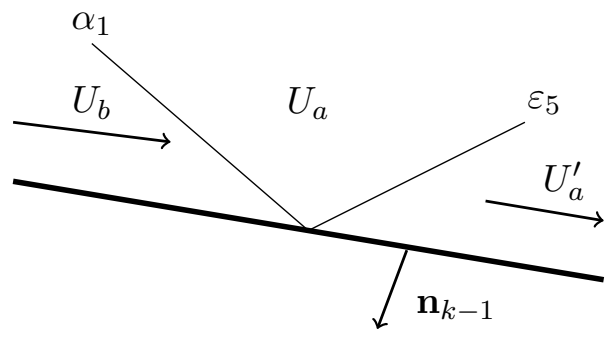

Fig. 4.4. Weak waves reflected and physical wave emerged at the boundary

Proof. By Lemma 4.1, there exists a $C^{2}$-function $\Psi$ such that

$$
U_{a}^{\prime}=\Psi\left(\varepsilon_{5} ; U_{a}\right) \quad \text { for }\left|\varepsilon_{5}\right| \ll 1 .
$$

Then problem (4.24) can be reduced to solving the equation:

$$
\Psi\left(\varepsilon_{5} ; \Phi_{1}\left(\alpha_{1} ; U_{b}\right)\right) \cdot\left(\mathbf{n}_{k-1}, 0,0,0\right)=0 .
$$

Thus, in a similar way as in Lemma 4.2, we obtain the existence of $\left(\varepsilon_{5}, U_{a}^{\prime}\right)$ and estimate (4.26). This completes the proof.

4.3. Wave-front tracking algorithm with fractional-step. In this subsection, we construct the approximate solutions to system (1.1) by combining the fractional-step technique with the wave-front tracking algorithm.

For given initial data $\bar{U}$, we define a sequence of piecewise constant functions $\bar{U}^{\nu}$ such that

$$
\left\|\bar{U}^{\nu}-\bar{U}\right\|_{L^{1}\left(R_{+}\right)}<2^{-\nu} \quad \text { for } \nu \in \mathbb{N}_{+} \text {and the mesh length } h>0 \text { defined above, }
$$


and approximate the wedge boundary as in $\S 4$. Then the $(\nu, h)$-approximate solution $U^{\nu, h}(x, y)$ is constructed as follows:

When $x=0$, all the Riemann solutions in $\bar{U}^{\nu}$ are obtained by the accurate Riemann solver, in which the number of wave-fronts is finite. Then, for fixed $h$, we construct the approximate solution $U^{\nu, h}$ in strip $0<x<h$, according to the ways as in $\S 4.1-4.2$ by solving the initial-boundary value problem. After that, we need to solve the nonhomogeneous problem on line $x=h$ for $k=1$. Inductively, assuming that the approximate solution $U^{\nu, h}(x, y)$ has been constructed for $0<x \leq(k-1) h$ and contains the jumps of shock fronts, weak rarefaction fronts, strong rarefaction fronts, and non-physical fronts, which are denoted by $\mathcal{J}=\mathcal{S} \cup \mathcal{R} \cup \mathcal{R}_{\mathrm{b}} \cup \mathcal{N} \mathcal{P}$, and the number of wave-fronts is finite in each interval $((m-1) h, m h)$ with $m \leq k-1$. Now we construct the approximate solution $U^{\nu, h}(x, y)$ for $(k-1) h<x \leq k h$. In $((k-1) h, k h)$, we solve the homogeneous problem (2.1) with initial data $U^{\nu, h}((k-1) h+, y)$. Since $U^{\nu, h}((k-1) h+, y)$ is piecewise constant and may be discontinuous on line $x=(k-1) h+$, then we solve the initial Riemann problem (4.1) and the lateral Riemann problem (4.9) with the initial data $U^{\nu, h}((k-1) h+, \cdot)$. The approximate solution $U^{\nu, h}(x, y)$ consists of a finite number of wave-fronts whose proof will be given in $\S 6$, for which some may interact at $x=\tau$ for $(k-1) h<\tau<k h$, and the corresponding Riemann problem is solved when two wave-fronts interact, or a wave-front hits the boundary. Then, owing to the reaction process, we solve the nonhomogeneous problem (2.1) from $x=k h-$ to $x=k h+$ with initial data $U^{\nu, h}(k h-, \cdot)$, that is,

$$
W\left(U^{\nu, h}(k h+, y)\right)=W\left(U^{\nu, h}(k h-, y)\right)+G\left(U^{\nu, h}(k h-, y)\right) h
$$

In each step of construction, we can change the speed of a single wave-front at a point by a quantity less than $2^{-\nu}$, so that no more than two wave-fronts interact, and that only one wave-front hits the boundary $\Gamma_{h, k-1}$ at the non-corner point, or only one wave is generated by the corner point and also only one wave crosses line $x=k h$. In order to avoid the number of wave-fronts to be infinite in a finite time for solving the homogeneous system (2.1), we also need to assign a generation order for each wave-front as stated in 4 . The outgoing wave-fronts are constructed and the generation orders are defined according to the following:

(i) All wave-fronts generated by the corner points can be constructed in the same way as in $§ 4.2$ and have order one.

(ii) A wave-front of order $k$ hits the boundary at the non-corner point $\left(\tau, g_{h}(\tau)\right)$. Then the generated lateral Riemann problem is solved by the accurate $\nu$-Riemann solver as in $\S 4.2$ and the generation order of the new wave from $\left(\tau, g_{h}(\tau)\right)$ is set to be $k+1$.

(iii) The $i_{1}$-wave-front $\alpha_{i_{1}}$ and $i_{2}$-wave front $\beta_{i_{2}}$, with order $n_{1}$ and $n_{2}$, respectively, interact at $\left(\tau, y_{\tau}\right)$ for some $y_{\tau}$. Assume that $\alpha_{i_{1}}$ lies below $\beta_{i_{2}}$. Then the new wave-fronts and their generation orders are given as follows:

(iii) ${ }_{1}$ For the case $n_{1}, n_{2}<\nu$, the outgoing wave-fronts generated from $\left(\tau, y_{\tau}\right)$ are constructed by the accurate Riemann solver, and the generation order of the $j$-th wave is 
given by

$$
\begin{cases}\max \left\{n_{1}, n_{2}\right\}+1, & j \neq i_{1}, i_{2}, \\ \min \left\{n_{1}, n_{2}\right\}, & j=i_{1}=i_{2}, \\ n_{1}, & j=i_{1} \neq i_{2}, \\ n_{2}, & j \neq i_{1}=i_{2} .\end{cases}
$$

(iii) $)_{2}$ If $\max \left\{n_{1}, n_{2}\right\}=\nu$, the outgoing wave-fronts generated from $\left(\tau, y_{\tau}\right)$ are given by the simplified Riemann solver, and the outgoing $i_{1}$-wave and $i_{2}$-wave are defined according to (4.6). The generation order of the outgoing non-physical wave-front is $\nu+1$.

(iii) $)_{3}$ If $n_{1}=\nu+1$ and $n_{2} \leq \nu$, then $\alpha_{i_{1}}$ is a non-physical wave-front. We use the simplified Riemann solver to construct the outgoing wave-fronts from $\left(\tau, y_{\tau}\right)$. The generation order of the outgoing non-physical wave-front is $\nu+1$, while the outgoing physical wave-front has the same generation order with $n_{2}$.

(iv) If the change of the angle of boundary is larger than $\frac{1}{\nu}$ and the weak wave is physical, then we employ the accurate Riemann solver to solve the Riemann problem. If the change of the angle of the boundary is less than $\frac{1}{\nu}$, then we ignore this perturbation.

(v) When a wave-front $\alpha$ of order $n$ hits line $x=k h$, then the new wave-fronts and their generation orders are defined as follows:

$(\mathrm{v})_{1}$ When $n \leq \nu$, the outgoing wave-fronts generated from the points on line $x=k h+$ are constructed by the accurate Riemann solver, and the generation order is defined as in (4.28).

$(\mathrm{v})_{2}$ When the incoming wave-front $\alpha$ is non-physical, then let it cross line $x=k h$ with the generation order unchanged.

$(\mathrm{v})_{3}$ For the strong rarefaction fronts cross line $x=k h$, its generation order is unchanged with order 1.

This completes the construction of the approximate solution.

In each step of construction, we have the following property for $Z^{\nu, h}(x, y)$ :

Lemma 4.4. If $0 \leq Z^{\nu, h}((k-1) h+, y) \leq 1$, then $Z^{\nu, h}(x, y)$ is unchanged when crossing the non-physical waves so that

$$
0 \leq Z^{\nu, h}(x, y) \leq 1
$$

for all $k \in \mathbb{N}_{+}$and $(x, y) \in((k-1) h, k h) \times\left(g_{h}(\tau), \infty\right)$.

Proof. We consider the Riemann problem with $Z^{\nu, h}(k h, y)$ as its initial data. In solving the accurate Riemann problem, we notice that $Z^{\nu, h}$ is unchanged when it crosses the $j$ wave-fronts, $j=1,5$, and $i$-contact discontinuities, $i=2,3$. The discontinuities of $Z^{\nu, h}$ propagate only along the 4 -contact discontinuities. Thus, $0 \leq Z^{\nu, h}(x, y) \leq 1$.

We now consider the simplified Riemann solution. Without loss of generality, suppose that $\alpha_{2(3,4)}$, which connects $\left\{U_{m}, U_{a}\right\}$, interacts with 5 -shock $\alpha_{5}$ with $\left\{U_{b}, U_{m}\right\}$ as its below and above states at $x=\tau$. Then it is solved by the simplified Riemann solver. 


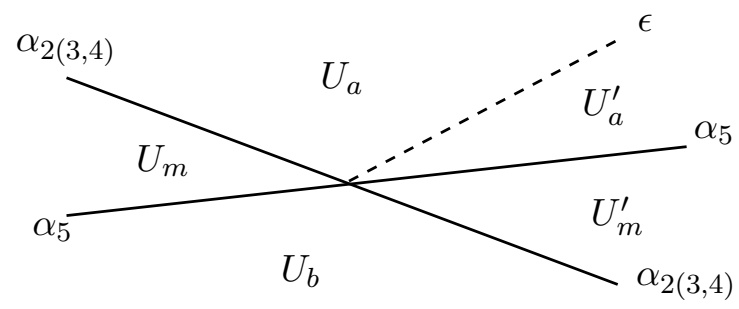

Fig. 4.5

Assume that $Z_{a}$ is the 5 th component of $U_{a}$, and also $Z_{m}, Z_{b}, Z_{m}^{\prime}, Z_{b}^{\prime}$ etc. denote the same meaning. Then, by the wave curves defined in $\S 2$, we have

$$
Z_{m}=Z_{b}, \quad Z_{a}=Z_{m}+\alpha_{4}, \quad Z_{m}^{\prime}=Z_{b}+\alpha_{4}, Z_{a}^{\prime}=Z_{m}^{\prime}
$$

which leads to $Z_{a}=Z_{a}^{\prime}=Z_{b}+\alpha_{4}$. This implies that $Z^{\nu+, h}(\tau, \cdot)$ is unchanged when it crosses the non-physical waves. Therefore,

$$
0 \leq Z^{\nu, h}(\tau+, y) \leq 1 \quad \text { for }(\tau, y) \in((k-1) h, k h) \times\left(g_{h}(\tau), \infty\right)
$$

This completes the proof.

At $x=k h$, we use (4.27) to obtain

$$
\begin{aligned}
& \rho_{+}^{\nu, h} u_{+}^{\nu, h}=\rho_{-}^{\nu, h} u_{-}^{\nu, h}, \\
& \rho_{+}^{\nu, h}\left(u_{+}^{\nu, h}\right)^{2}+p_{+}^{\nu, h}=\rho_{-}^{\nu, h}\left(u_{-}^{\nu, h}\right)^{2}+p_{-}^{\nu, h}, \\
& \rho_{+}^{\nu, h} u_{+}^{\nu, h} v_{+}^{\nu, h}=\rho_{-}^{\nu, h} u_{-}^{\nu, h} v_{-}^{\nu, h}, \\
& \rho_{+}^{\nu, h} u_{+}^{\nu, h}\left(\tilde{h}_{+}^{\nu, h}+\frac{\left(u_{+}^{\nu, h}\right)^{2}+\left(v_{+}^{\nu, h}\right)^{2}}{2}\right) \\
& \quad=\rho_{-}^{\nu, h} u_{-}^{\nu, h}\left(\tilde{h}_{-}^{\nu, h}+\frac{\left(u_{-}^{\nu, h}\right)^{2}+\left(v_{-}^{\nu, h}\right)^{2}}{2}\right)+\tilde{q} \rho_{-}^{\nu, h} Z_{-}^{\nu, h} \phi\left(T_{-}^{\nu, h}\right) h, \\
& \rho_{+}^{\nu, h} u_{+}^{\nu, h} Z_{+}^{\nu, h}=\rho_{-}^{\nu, h} u_{-}^{\nu, h} Z_{-}^{\nu, h}-\rho_{-}^{\nu, h} Z_{-}^{\nu, h} \phi\left(T_{-}^{\nu, h}\right) h,
\end{aligned}
$$

where $U_{+}^{\nu, h}$ and $U_{-}^{\nu, h}$ denote $U^{\nu, h}(x, y)$ taking values at $x=k h \pm$, respectively.

For equations (4.30)-(4.34), we have the following properties that indicate the change in $U^{\nu, h}$ due to the reaction process. 
Lemma 4.5. For $h>0$ sufficiently small, there exist positive constants $L$ and $\bar{K}$, independent of $(\nu, h)$, such that

$$
\begin{aligned}
& T^{\nu, h}(k h+, y) \geq T^{\nu, h}(k h-, y) \geq \bar{K}>0, \\
& u^{\nu, h}(k h+, y)-u^{\nu, h}(k h-, y)=O(1)\|\bar{Z}\|_{\infty} e^{-L k h} h, \\
& v^{\nu, h}(k h+, y)-v^{\nu, h}(k h-, y)=0, \\
& p^{\nu, h}(k h+, y)-p^{\nu, h}(k h-, y)=O(1)\|\bar{Z}\|_{\infty} e^{-L k h} h, \\
& \rho^{\nu, h}(k h+, y)-\rho^{\nu, h}(k h-, y)=O(1)\|\bar{Z}\|_{\infty} e^{-L k h} h, \\
& Z^{\nu, h}(k h+, y)-Z^{\nu, h}(k h-, y)=O(1)\|\bar{Z}\|_{\infty} e^{-L k h} h .
\end{aligned}
$$

In addition, for $k \in \mathbf{N}_{+}$and $y \geq g_{h}(k h)$,

$$
\begin{aligned}
& 0 \leq Z^{\nu, h}(k h+, y) \leq Z^{\nu, h}(k h-, y) e^{-L h}, \\
& 0 \leq Z^{\nu, h}(k h+, y) \leq O(1)\|\bar{Z}\|_{\infty} e^{-L k h} h \leq 1 .
\end{aligned}
$$

Proof. We carry out the proof by induction on $k$.

For $k=0$, by $\left(\mathbf{H}_{\mathbf{2}}\right)$, we have

$$
T^{\nu, h}(0+, y)=\bar{T}>T^{*}>0, \quad 0 \leq Z^{\nu, h}(0+, y)=\bar{Z} \leq 1 .
$$

Suppose that (4.35)-(4.36) hold for $k=l-1$. We now prove (4.35)-(4.36) for $k=l$. To see this, for $h>0$ sufficiently small, we first have

$$
\begin{aligned}
& T^{\nu, h}(l h+, y)-T^{\nu, h}(l h-, y) \\
& =\frac{(\gamma-1) \tilde{q}\left(\left(u^{\nu, h}\right)^{2}-\frac{c^{2}\left(T^{\nu, h}\right)}{\gamma}\right)(l h-, y)}{R \rho^{\nu, h} u^{\nu, h}\left(\left(u^{\nu, h}\right)^{2}-c^{2}\left(T^{\nu, h}\right)\right)(l h-, y)}\left(\rho^{\nu, h} Z^{\nu, h} \phi\left(T^{\nu, h}\right)\right)(l h-, y) h+O\left(h^{2}\right) .
\end{aligned}
$$

Since $\left(u^{\nu, h}\right)^{2}(k h-, y)>c^{2}\left(T^{\nu, h}(k h-, y)\right)$ and $\gamma>1$, then

$$
T^{\nu, h}(k h+, y) \geq T^{\nu, h}(k h-, y),
$$

which shows that the temperature $T^{\nu, h}$ does not decrease owing to the reaction process. Then, by the induction hypothesis, there exists a constant $\bar{K}$ independent of $(\nu, h)$ such that the first estimate in (4.35) holds.

By (4.30) and (4.34), we have

$$
Z^{\nu, h}(l h+, y)=\left(1-\frac{\phi\left(T^{\nu, h}\right)(l h-, y)}{u^{\nu, h}(l h-, y)} h\right) Z^{\nu, h}(l h-, y) .
$$

Since $\phi(T)$ is assumed to be Lipschitz continuous, nonnegative, and increasing, then there exists a constant $L>0$ such that

$$
Z^{\nu, h}(l h+, y)-Z^{\nu, h}(l h-, y) \leq-Z^{\nu, h}(l h-, y) L h,
$$

which implies that

$$
Z^{h}(l h+, y) \leq Z^{\nu, h}(l h-, y)(1-L h) \leq Z^{\nu, h}(l h-, y) e^{-L h} .
$$


On the other hand, by Lemma 4.4, we have

$$
Z^{\nu, h}(l h-, y) \leq Z^{h}((l-1) h+, y) .
$$

Then, by induction,

$$
Z^{h}(l h-, y) \leq\|\bar{Z}\|_{\infty} e^{-L(l-1) h} O(h) .
$$

Thus, for $h$ sufficiently small, we have

$$
Z^{h}(l h+, y) \leq Z^{\nu, h}(l h-, y) e^{-L h} \leq Z^{\nu, h}((l-1) h+, y) e^{-L h} \leq\|\bar{Z}\|_{\infty} e^{-L l h} O(h),
$$

which leads to the last estimate in (4.35) and the esimates in (4.36).

To prove the remaining part of (4.35), let $U_{+}^{\nu, h}=\left(u_{+}^{\nu, h}, v_{+}^{\nu, h}, p_{+}^{\nu, h}, \rho_{+}^{\nu, h}, Z_{+}^{\nu, h}\right)$ and $U_{-}^{\nu, h}=$ $\left(u_{-}^{\nu, h}, v_{-}^{\nu, h}, p_{-}^{\nu, h}, \rho_{-}^{\nu, h}, Z_{-}^{\nu, h}\right)$ denote $U^{\nu, h}(x, y)$ taking values at $x=k h \pm$, respectively, and also $T_{ \pm}^{\nu, h}=T^{\nu, h}(l h \pm, y)$. Then, by (4.30) (4.32), we have

$$
u_{+}^{\nu, h}=\frac{\rho_{-}^{\nu, h} u_{-}^{\nu, h}}{\rho_{+}^{\nu, h}}, \quad v_{+}^{\nu, h}=v_{-}^{\nu, h}, \quad p_{+}^{\nu, h}=p_{-}^{\nu, h}+\rho_{-}^{\nu, h}\left(u_{-}^{\nu, h}\right)^{2}-\frac{\left(\rho_{-}^{\nu, h} u_{-}^{\nu, h}\right)^{2}}{\rho_{+}^{\nu, h}} .
$$

Substitute $\left(u_{+}^{\nu, h}, v_{+}^{\nu, h}, p_{+}^{\nu, h}\right)$ into (4.33), we have

$$
\begin{aligned}
& \frac{\left(\rho_{-}^{\nu, h} u_{-}^{\nu, h}\right)^{2}}{\left(\rho_{+}^{\nu, h}\right)^{2}}-\frac{2 \gamma\left(\rho_{-}^{\nu, h}\left(u_{-}^{\nu, h}\right)^{2}+p_{-}^{\nu, h}\right)}{(\gamma+1) \rho_{+}^{\nu, h}}+\frac{2 \gamma+p_{-}^{\nu, h}+(\gamma-1) \rho_{-}^{\nu, h}\left(u_{-}^{\nu, h}\right)^{2}}{(\gamma+1) \rho_{-}^{\nu, h}} \\
& \quad+\frac{2(\gamma-1) \tilde{q} Z_{-}^{\nu, h} \phi\left(T_{-}^{\nu, h}\right)}{(\gamma+1) u_{-}^{\nu, h}} h=0 .
\end{aligned}
$$

Therefore, we obtain

$$
\frac{1}{\rho_{+}^{\nu, h}}=\frac{\gamma\left(\rho_{-}^{\nu, h}\left(u_{-}^{\nu, h}\right)^{2}+p_{-}^{\nu, h}\right)+\sqrt{\left(\rho_{-}^{\nu, h}\left(u_{-}^{\nu, h}\right)^{2}-\gamma p_{-}^{\nu, h}\right)^{2}-2 \tilde{q}\left(\gamma^{2}-1\right)\left(\rho_{-}^{\nu, h}\right)^{2} u_{-}^{\nu, h} Z_{-}^{\nu, h} \phi\left(T_{-}^{\nu, h}\right)}}{(\gamma+1)\left(\rho_{-}^{\nu, h} u_{-}^{\nu, h}\right)^{2}} .
$$

Using the Taylor expansion, we have

$$
\frac{1}{\rho_{+}^{\nu, h}}=\frac{1}{\rho_{-}^{\nu, h}}+Z_{-}^{\nu, h} O(h)
$$

By (4.36), it follows that

$$
\rho_{+}^{\nu, h}-\rho_{-}^{\nu, h}=\|\bar{Z}\|_{\infty} e^{-L l h} O(h) .
$$

For $u_{+}^{\nu, h}$, we have

$$
u_{+}^{\nu, h}-u_{-}^{\nu, h}=\left(\frac{\rho_{-}^{\nu, h}}{\rho_{+}^{\nu, h}}-1\right) u_{-}^{\nu, h}
$$

which leads to the desire result for $u_{+}^{\nu, h}$.

Similar arguments also apply to $p_{+}^{\nu, h}$. This completes the proof. 


\section{Glimm-Type Functional}

In this section, we denote $U^{\nu, h}(x, y)$ as the $(\nu, h)$-approximate solution constructed by the fractional-step wave-front tracking method as in $\S 3$. On every interval $((k-$ $1) h, k h), k \geq 1, U^{\nu, h}(x, y)$ consists of shocks, rarefaction fronts, and non-physical fronts. We distinguish strong 5-rarefaction wave-fronts from the others as follows:

Definition 5.1. A wave-front $s$ in $U^{\nu, h}(x, y)$ is said to be a front of the strong rarefaction wave, provided that $s$ is a 5-rarefaction wave-front with order 1 . If a wave $\alpha$ is a shock or 5-rarefaction wave-front with the generation order $n \geq 2$, or $\alpha$ is a non-physical front, then $\alpha$ is called a weak wave.

Let $y_{\alpha}(t)$ and $\alpha(t)$ be the location and magnitude for any weak wave $\alpha$ in $U^{\nu, h}(x, y)$ on line $x=t$ respectively. For a front of the strong rarefaction wave, denote the magnitude and location of $s$ on $x=t$ by $s(t)$ and $Y_{s}(t)$, respectively. In addition, define

$$
\Omega_{\mathrm{Ra}}(x)=\left\{\omega\left(A_{k}\right): \omega\left(A_{k}\right) \leq 0, A_{k}=\left(k h, g_{h}(k h)\right), k h \geq x\right\},
$$

where $\omega\left(A_{k}\right)=\theta_{k}-\theta_{k-1}$ and $R a$ stands for the strong rarefaction wave.

We now introduce some weights for the weak waves as follows: For any $i$-weak wave $\alpha_{i}, 1 \leq i \leq 4$, and any non-physical wave $\epsilon$ at a non-interaction location $x$, let

$$
\begin{aligned}
& R\left(x, \alpha_{i},-\right)=\left\{s: s \text { is a front of strong rarefaction wave with } Y_{s}(x) \leq y_{\alpha_{i}}(x)\right\}, \\
& R(x, \epsilon,+)=\left\{s: s \text { is a front of strong rarefaction wave with } Y_{s}(x) \geq y_{\epsilon}(x)\right\}
\end{aligned}
$$

and, for $K_{\mathrm{b}}>0, K_{\mathrm{w}}>0$, and $K_{\mathrm{np}}>0$, define

$$
\begin{aligned}
& W\left(\alpha_{i}, x,-\right)=\exp \left(K_{\mathrm{b}} \sum\left\{|\omega|: \omega \in \Omega_{\mathrm{Ra}}(x)\right\}+K_{\mathrm{w}} \sum\left\{|s|: s \in R\left(x, \alpha_{i},-\right)\right\}\right), \\
& W(\epsilon, x,+)=\exp \left(K_{\mathrm{np}} \sum\{|s|: s \in R(x, \epsilon,+)\}\right) .
\end{aligned}
$$

Definition 5.2 (Approaching waves). We say that two weak wave-fronts $\alpha$ and $\beta$, located at points $x_{\alpha}<x_{\beta}$ and belonging to the characteristic families $i_{\alpha}, i_{\beta} \in\{1,2, \cdots, 6\}$, respectively, approach each other if either $i_{\alpha}>i_{\beta}$ or $i_{\alpha}=i_{\beta}$, and at least one of them is a shock. In this case, we write $(\alpha, \beta) \in \mathcal{A}$.

Then we have

Definition 5.3. The strengths of weak waves in $U^{h}(x, y)$ are defined by

$$
\begin{aligned}
L_{\mathrm{w}}^{j}(U ; x) & =\sum\left\{\left|\alpha_{j}\right|: \alpha_{j} \text { is a weak and physical } j \text {-wave }\right\}, \quad 1 \leq j \leq 5, \\
L_{\mathrm{np}}(U ; x) & =\sum\{|\epsilon|: \epsilon \text { is a non-physical wave }\}
\end{aligned}
$$


and the wave potentials are defined by

$$
\begin{aligned}
& Q_{0}(U ; x)=\sum\left\{\left|\alpha_{i}\right|\left|\beta_{j}\right|: \alpha_{i} \text { and } \beta_{j} \text { approach weak waves }\right\}, \\
& Q_{\mathrm{Bi}}(U ; x)=\sum\left\{W\left(\alpha_{i}, x,-\right)\left|\alpha_{i}\right|: \alpha \text { is a weak } i \text {-wave }\right\}, \quad 1 \leq i \leq 4, \\
& Q_{\mathrm{B} 5}(U ; x)=\sum\left\{\left|\alpha_{5}\right|: \alpha_{5} \text { is a } 5 \text {-weak wave }\right\}, \\
& Q_{\mathrm{BNP}}(U ; x)=\sum\{W(\epsilon, x,+)|\epsilon|: \epsilon \text { is a non-physical wave }\}, \\
& Q_{\mathrm{c}}(U ; x)=\sum\left\{\left|\omega\left(A_{k}\right)\right|: A_{k}=\left(k h, g_{h}(k h)\right) \text { with } \omega\left(A_{k}\right)>0 \text { for } k h>x\right\} .
\end{aligned}
$$

In order to measure the amounts of the 5-rarefaction waves expanding before $x$, we introduce the following:

Definition 5.4. For $x \notin\left\{k h: k \in \mathbf{N}_{+}\right\}$, define

$$
F_{1}(U ; x)=\left|T V \cdot\left\{\theta(U(x, \cdot)) ;\left[g_{h}(x), \infty\right)\right\}-\hat{\theta}(x)\right|,
$$

where

$\hat{\theta}(x)=\sum\left\{\left|\omega\left(A_{k}\right)\right|: A_{k}\right.$ is a corner point $\left(k h, g_{h}(k h)\right)$ with $\omega\left(A_{k}\right) \leq 0$ for $\left.k h<x\right\}$.

Finally, we turn to the construction of the Glimm-type functional at the non-interaction point $x$.

Definition 5.5. Define

$$
\begin{aligned}
& L_{\mathrm{w}}(U ; x)=\sum_{i=1}^{5} L_{\mathrm{w}}^{i}(U ; x)+L_{\mathrm{np}}(U ; x), \\
& Q(U ; x)=K_{0} Q_{0}(U ; x)+K_{1} \sum_{i=1}^{4} Q_{\mathrm{Bi}}(U ; x)+Q_{\mathrm{B} 5}(U ; x)+Q_{\mathrm{BNP}}(U ; x)+K_{\mathrm{c}} Q_{\mathrm{c}}(U ; x), \\
& F_{0}(U ; x)=L_{\mathrm{w}}(U ; x)+K Q(U ; x), \\
& F(U ; x)=F_{1}(U ; x)+C_{*} F_{0}(U ; x),
\end{aligned}
$$

where $C_{*}, K, K_{0}, K_{1}$, and $K_{\mathrm{c}}$ are positive constants specified later.

\section{Estimates of THE NON-REACTING STEP}

In this section, our main task is to prove the decreasing of the Glimm-type functional for the homogeneous system (2.1) by the induction procedure. To this end, let $U^{\nu, h}(x, y)$ be the approximate solution of system (2.1) constructed above. For any $(k-1) h<\tau<k h$, we make the following inductive assumptions:

$A_{1}(\tau-)$ : Before $\tau$, the strength of every front is less than $\delta_{*}$;

$$
A_{2}(\tau-):\left.U^{\nu, h}(x, \cdot)\right|_{x<\tau} \in D_{\delta_{* *}}\left(U_{\infty}\right)
$$

where $\delta_{*} \in\left(0, \delta_{0}\right)$ and $\delta_{* *} \in\left(0, \min \left\{\delta_{0}, \delta_{1}\right\}\right)$ are well chosen such that the Riemann problems considered in $\S 4$ with the initial data satisfying $A_{1}(\tau-)-A_{2}(\tau-)$ are solvable. 
$A_{3}(\tau-)$ : For any $0<\delta_{0 *}<\frac{1}{2}\left(\lim _{x \rightarrow \infty} g_{*}^{\prime}(x+)-\theta_{\text {crit }}\right)$,

$$
\sum_{k}\left|\omega\left(A_{k}\right)\right| \leq-\theta_{\text {crit }}-\delta_{0 *}
$$

where $\theta_{\text {crit }}$ is a critical angle given in (2.17).

In order to obtain the global interaction estimates and the bound on the amount of strong rarefaction wave-fronts, we need some auxiliary lemmas whose proofs are similar to [45].

Lemma 6.1. There is $\delta_{*}^{\prime}>0$ such that function $\theta\left(\Phi_{5}\left(\alpha_{5}, U\right)\right)$ is a strictly increasing function of $\alpha_{5}$ in $\left\{\alpha_{5}: \alpha_{5} \geq-\delta_{*}^{\prime}, \Phi_{5}\left(\alpha_{5}, U\right) \in D_{\delta_{0}}\left(U_{\infty}\right)\right\}$ for any $U \in D_{\delta_{0}}\left(U_{\infty}\right)$. Moreover, there exist two positive constants $C_{1}$ and $C_{2}$ such that

$$
C_{1}\left|\alpha_{5}\right| \leq\left|\theta\left(\Phi_{5}\left(\alpha_{5}, U\right)\right)-\theta(U)\right| \leq C_{2}\left|\alpha_{5}\right| \text {. }
$$

Proof. For $\alpha_{5} \geq 0$, by Lemma 2.1 and Lemma 10.1 in the appendix, we have

$$
\begin{aligned}
\left.\frac{\partial \theta(\tilde{U})}{\partial \alpha_{5}}\right|_{\tilde{U}=\Phi_{5}\left(\alpha_{5}, U\right)} & =\left.\left(\nabla_{\tilde{U}} \theta(\tilde{U}) \cdot \frac{\partial \tilde{U}}{\partial \alpha_{5}}\right)\right|_{\tilde{U}=\Phi_{5}\left(\alpha_{5}, U\right)} \\
& =\left.\frac{\kappa_{5}(\tilde{U}) \cos \theta_{\mathrm{ma}}(\tilde{U})}{q(\tilde{U}) \cos \left(\theta(\tilde{U})+\theta_{\mathrm{ma}}(\tilde{U})\right)}\right|_{\tilde{U}=\Phi_{5}\left(\alpha_{5}, U\right)}>0
\end{aligned}
$$

for any $\Phi_{5}\left(\alpha_{5}, U\right) \in D_{\delta_{0}}\left(U_{\infty}\right)$ and $U \in D_{\delta_{0}}\left(U_{\infty}\right)$. A similar proof works for $-\delta_{*}^{\prime} \leq \alpha_{5}<0$. This completes the proof.

Lemma 6.2. Suppose that $U_{1}, U_{2}, U_{3} \in D_{\delta_{0}}\left(U_{\infty}\right)$ are three constant states such that $\left\{U_{1}, U_{2}\right\}$ are connected by $U_{2}=\Phi\left(\alpha_{5}, \alpha_{4}, \alpha_{3}, \alpha_{2}, \alpha_{1} ; U_{1}\right)$ and $\left\{U_{2}, U_{3}\right\}$ are connected by a non-physical wave-front with strength $\epsilon$. Then

$$
\left\{\begin{array}{l}
\left|\left(J\left(q, \frac{q^{2}}{2}+\frac{c^{2}}{\gamma-1}\right)+\theta\right)\left(U_{3}\right)-\left(J\left(q, \frac{q^{2}}{2}+\frac{c^{2}}{\gamma-1}\right)+\theta\right)\left(U_{1}\right)\right| \\
=O(1)\left(\sum_{i=1}^{4}\left|\alpha_{i}\right|+\left|\alpha_{5}^{-}\right|+|\epsilon|\right), \\
\left|\left(\frac{q^{2}}{2}+\frac{c^{2}}{\gamma-1}\right)\left(U_{3}\right)-\left(\frac{q^{2}}{2}+\frac{c^{2}}{\gamma-1}\right)\left(U_{1}\right)\right|=O(1)\left(\sum_{i=1}^{4}\left|\alpha_{i}\right|+\left|\alpha_{5}^{-}\right|+|\epsilon|\right) \\
\left|\frac{p_{3}}{\rho_{3}^{\gamma}}-\frac{p_{1}}{\rho_{1}^{\gamma}}\right|=O(1)\left(\sum_{i=1}^{4}\left|\alpha_{i}\right|+\left|\alpha_{5}^{-}\right|+|\epsilon|\right) \\
\left|Z_{3}-Z_{1}\right|=O(1)\left(\sum_{i=1}^{4}\left|\alpha_{i}\right|+\left|\alpha_{5}^{-}\right|+|\epsilon|\right)
\end{array}\right.
$$

where $\alpha_{5}^{-}=\min \left\{\alpha_{5}, 0\right\}$, and the bound of $O(1)$ is independent of $U_{k}, 1 \leq k \leq 3$.

Proof. By the mean-value theorem and the fact that, for $\alpha_{5}>0$,

$$
\begin{aligned}
& \left(J\left(q, \frac{q^{2}}{2}+\frac{c^{2}}{\gamma-1}\right)+\theta\right)\left(\Phi_{5}\left(\alpha_{5}, U\right)\right)=\left(J\left(q, \frac{q^{2}}{2}+\frac{c^{2}}{\gamma-1}\right)+\theta\right)(U), \\
& \left(\frac{q^{2}}{2}+\frac{c^{2}}{\gamma-1}\right)\left(\Phi_{5}\left(\alpha_{5}, U\right)\right)=\left(\frac{q^{2}}{2}+\frac{c^{2}}{\gamma-1}\right)(U),
\end{aligned}
$$

we conclude the proof. 
Let $\alpha_{i}$ and $\beta_{j}, 1 \leq i, j \leq 5$, be the approaching weak waves, and let $\epsilon$ be a non-physical wave. Let $s$ denote the strong wave-front and $\omega_{k-1}>0$ for some $k \geq 1$. Define

$$
E_{\nu, h}(\tau)= \begin{cases}\left|\alpha_{i}\right|\left|\beta_{j}\right|, & \left(\alpha_{i}, \beta_{j}\right) \in \mathcal{A}, 1 \leq i, j \leq 5, \\ \left|\alpha_{i}\right||\epsilon|, & 1 \leq i \leq 5, \\ \left|\alpha_{i}\right||s|, & 1 \leq i \leq 5, \\ \left|\alpha_{1}\right|, & \\ \left|\omega_{k-1}\right|, & \omega_{k-1}>0, \quad k \geq 1, \\ \left|\alpha_{5}\right|, & \end{cases}
$$

where $\tau \in((k-1) h, k h)$ act as the times when two waves interact or a weak wave reflects at a non-corner point, or weak waves generated by the corner points. Here we assume that only one interaction occurs at $x=\tau$. Then, for the non-reacting flow past a bending wall, we have the following proposition.

Proposition 6.1. Assume that the hypotheses $A_{1}(\tau-)-A_{2}(\tau-)$ hold for any $(k-1) h<$ $\tau<k h$. Then there exist positive constants $\delta_{2}, C_{*}, K_{0}, K_{1}, K_{2}$, and $K_{\mathrm{c}}$, independent of $(\nu, \tau)$, such that, if $F\left(U^{\nu, h} ; \tau-\right)<\delta_{2}$,

$$
\begin{aligned}
& F\left(U^{\nu, h} ; \tau+\right) \leq F\left(U^{\nu, h} ; \tau-\right)-\frac{1}{4} E_{\nu, h}(\tau), \\
& Q\left(U^{\nu, h} ; \tau+\right) \leq Q\left(U^{\nu, h} ; \tau-\right)-\frac{1}{4} E_{\nu, h}(\tau) .
\end{aligned}
$$

Moreover, for $(k-1) h<x<k h$, the following hold:

(i) Before $\tau_{1}$, the strength of every weak wave-front is less than $\delta_{*}$;

(ii) There is a constant $C_{3}>0$ independent of $(\nu, h)$ such that

$$
\left|s_{0}(x)\right| \leq \frac{C_{3}}{\nu}
$$

for any strong rarefaction front $s_{0}(x)$;

(iii) For given $\nu \in \mathbb{N}_{+}$sufficiently large, the number of wave-fronts in $U^{\nu, h}(x, y)$ is finite, and the total amount of a non-physical wave $\epsilon_{0}(x)$ is of order $O(1) 2^{-\nu}$, that is, there exists a constant $C_{\mathrm{np}}>0$ such that

$$
\sum_{\epsilon_{0}}\left|\epsilon_{0}(x)\right| \leq \frac{C_{\mathrm{np}}}{2^{\nu}} \quad \text { for } x<\tau_{1},
$$

where $\tau_{1}<k h$ is the point next to $\tau$ such that the interaction occurs on $x=\tau_{1}$.

Proof. Without loss of generality, we assume that there is only one interaction of waves at $x=\tau$. We now focus only on the following three cases, since the others are similar to [45.

Case 1. Strong rarefaction wave and weak 2-wave interaction. Suppose that a front of strong rarefaction wave $s_{0}$ with $\left\{U_{b}, U_{m}\right\}$ as its below and above states interacts with a weak wave-front $\alpha_{2}$ that connects $\left\{U_{m}, U_{a}\right\}$ on line $x=\tau$. Let the outgoing waves be denoted by $s_{0}^{\prime}$ and $\gamma_{j}, 1 \leq j \leq 4$, and $\epsilon$ be the nonphysical wave, and let $\left\{U_{m 1}^{\prime}, U_{m 2}^{\prime}, U_{a}^{\prime}\right\}$ 
be denoted as the constant states between $\gamma_{1}$ and $\gamma_{2(3,4)}, \gamma_{2(3,4)}$ and $s_{0}^{\prime}$, and $s_{0}^{\prime}$ and $\epsilon$, respectively. Then, by the Glimm interaction estimates, we have

$$
\begin{aligned}
& \gamma_{2}=\alpha_{2}+O(1)\left|\alpha_{2}\right|\left|s_{0}\right|, \quad \gamma_{j}=O(1)\left|\alpha_{2}\right|\left|s_{0}\right|, \quad j=1,3,4, \\
& s_{0}^{\prime}=s_{0}+O(1)\left|\alpha_{2}\right|\left|s_{0}\right|, \quad \epsilon=O(1)\left|\alpha_{2}\right|\left|s_{0}\right|,
\end{aligned}
$$

where the bound of $O(1)$ depends only on the system. With the above estimates, we conclude

$$
\begin{aligned}
& L_{\mathrm{w}}\left(U^{\nu, h} ; \tau+\right)-L_{\mathrm{w}}\left(U^{\nu, h} ; \tau-\right)=O(1)\left|\alpha_{2}\right|\left|s_{0}\right|, \\
& K_{0} Q_{0}\left(U^{\nu, h} ; \tau+\right)+Q_{B 5}\left(U^{\nu, h} ; \tau+\right)+Q_{B N P}\left(U^{\nu, h} ; \tau+\right)+K_{\mathrm{c}} Q_{\mathrm{c}}\left(U^{\nu, h} ; \tau+\right) \\
& \quad \quad-K_{0} Q_{0}\left(U^{\nu, h} ; \tau-\right)-Q_{B 5}\left(U^{\nu, h} ; \tau-\right)-Q_{B N P}\left(U^{\nu, h} ; \tau-\right)-K_{\mathrm{c}} Q_{\mathrm{c}}\left(U^{\nu, h} ; \tau-\right) \\
& \quad=O(1)\left|\alpha_{2}\right|\left|s_{0}\right| L_{\mathrm{w}}\left(U^{\nu, h} ; \tau-\right),
\end{aligned}
$$

and, for $K_{\mathrm{w}}$ sufficiently large and $L_{\mathrm{w}}\left(U^{\nu, h} ; \tau-\right)$ sufficiently small,

$$
\begin{aligned}
& \sum_{1 \leq i \leq 4} Q_{B i}\left(U^{\nu, h} ; \tau+\right)-\sum_{1 \leq i \leq 4} Q_{B i}\left(U^{\nu, h} ; \tau-\right) \\
& =\left|\alpha_{2}\right| W\left(\alpha_{2}, \tau-,-\right)\left(\left(1+O(1)\left|s_{0}\right|\right) e^{-K_{\mathrm{w}}\left|s_{0}\right|}-1\right) \\
& \quad+\sum_{1 \leq i \leq 4} \sum_{\beta_{i} \neq \gamma_{i}}\left|\beta_{i}\right| W\left(\beta_{i},-, \tau-\right)\left(e^{O(1) K_{\mathrm{w}}\left|\alpha_{2}\right|\left|s_{0}\right|}-1\right) \\
& \leq-\frac{K_{\mathrm{w}}}{2}\left|\alpha_{2}\right|\left|s_{0}\right|,
\end{aligned}
$$

which leads to

$$
Q\left(U^{\nu, h} ; \tau+\right)-Q\left(U^{\nu, h} ; \tau-\right) \leq-\frac{K_{\mathrm{w}}}{2}\left|\alpha_{2}\right|\left|s_{0}\right|
$$

To estimate $F_{1}\left(U^{\nu, h}, \tau+\right)$, let

$$
\theta(\Phi(\alpha ; U))=f\left(\alpha_{5}, \alpha_{2(3,4)}, \alpha_{1} ; U\right) .
$$

Then we have

$$
\begin{aligned}
& \theta\left(U_{a}^{\prime}\right)-\theta\left(U_{m 2}^{\prime}\right)-\theta\left(U_{m}\right)+\theta\left(U_{b}\right) \\
& =f\left(s_{0}^{\prime}, \gamma_{2(3,4)}, \gamma_{1} ; U_{b}\right)-f\left(0, \gamma_{2(3,4)}, \gamma_{1} ; U_{b}\right)-f\left(s_{0}, 0,0 ; U_{b}\right)+f\left(0,0,0 ; U_{b}\right) \\
& =f\left(s_{0}, \alpha_{2}, 0 ; U_{b}\right)-f\left(0, \alpha_{2}, 0 ; U_{b}\right)-f\left(s_{0}, 0,0 ; U_{l}\right)+f\left(0,0,0 ; U_{b}\right)+O(1)\left|\alpha_{2}\right|\left|s_{0}\right| \\
& =O(1)\left|\alpha_{2}\right|\left|s_{0}\right| .
\end{aligned}
$$

Similarly,

$$
\theta\left(U_{m 1}^{\prime}\right)-\theta\left(U_{b}\right)-\theta\left(U_{a}\right)+\theta\left(U_{m}\right)=O(1)\left|\alpha_{2}\right|\left|s_{0}\right|, \quad \theta\left(U_{a}\right)-\theta\left(U_{a}^{\prime}\right)=O(1)\left|\alpha_{2}\right|\left|s_{0}\right| .
$$


Then, by Lemma 6.1, we have

$$
\begin{aligned}
& T V \cdot\left\{\theta\left(U^{\nu, h}(\tau+, \cdot)\right) ;\left[g_{h}(\tau+), \infty\right)\right\}-T V \cdot\left\{\theta\left(U^{\nu, h}(\tau-, \cdot)\right) ;\left[g_{h}(\tau-), \infty\right)\right\} \\
&=\left|\theta\left(U_{a}\right)-\theta\left(U_{a}^{\prime}\right)\right|+\left|\theta\left(U_{a}^{\prime}\right)-\theta\left(U_{m 2}^{\prime}\right)\right|+\left|\theta\left(U_{m 1}^{\prime}\right)-\theta\left(U_{b}\right)\right| \\
&-\left|\theta\left(U_{a}\right)-\theta\left(U_{m}\right)\right|-\left|\theta\left(U_{m}\right)-\theta\left(U_{b}\right)\right| \\
&= O(1)\left|\alpha_{2}\right|\left|s_{0}\right|,
\end{aligned}
$$

where we have used the fact that $\theta\left(U_{m 2}^{\prime}\right)=\theta\left(U_{m 1}^{\prime}\right)$. Therefore, we obtain

$$
F_{1}\left(U^{\nu, h} ; \tau+\right)-F_{1}\left(U^{\nu, h} ; \tau-\right)=O(1)\left|\alpha_{2}\right|\left|s_{0}\right| .
$$

Finally, with the estimates on $L_{\mathrm{w}}\left(U^{\nu, h} ; \tau+\right), Q\left(U^{\nu, h} ; \tau+\right)$, and $F_{1}\left(U^{\nu, h} ; \tau+\right)$, and choosing $K$ and $C_{*}$ sufficiently large, we conclude

$$
F\left(U^{\nu, h} ; \tau+\right)-F\left(U^{\nu, h} ; \tau-\right) \leq-\frac{1}{4}\left|\alpha_{2}\right|\left|s_{0}\right| .
$$

Case 2. Reflection at the approximate boundary. Let a weak wave $\alpha_{1}$ that hits the approximate boundary at a non-corner point $\left(\tau, g_{h}(\tau)\right)$ be the outgoing wave. Then, by Lemma 4.3, we have

$$
\varepsilon_{5}=O(1) \alpha_{1},
$$

where the bound of $O(1)$ depends only on the system. Moreover, for $L_{\mathrm{w}}\left(U^{\nu, h} ; \tau-\right)$ sufficiently small and $K_{1}$ sufficiently large, we have

$$
\begin{aligned}
& L_{\mathrm{w}}\left(U^{\nu, h} ; \tau+\right)-L_{\mathrm{w}}\left(U^{\nu, h} ; \tau-\right)=-\left|\alpha_{1}\right|+O(1)\left|\alpha_{1}\right|, \\
& Q\left(U^{\nu, h} ; \tau+\right)-Q\left(U^{\nu, h} ; \tau-\right) \leq-\frac{1}{2}\left|\alpha_{1}\right|, \\
& F_{1}\left(U^{\nu, h} ; \tau+\right)-F_{1}\left(U^{\nu, h} ; \tau-\right)=O(1)\left|\alpha_{1}\right| .
\end{aligned}
$$

Therefore, we can choose $K$ and $C_{*}$ sufficiently large to obtain

$$
F\left(U^{\nu, h} ; \tau+\right)-F\left(U^{\nu, h} ; \tau-\right) \leq-\frac{1}{4}\left|\alpha_{1}\right| .
$$

Case 3. New waves generated by the corner points. We now consider the non-reaction flow past a corner point $A_{k-1}=\left((k-1) h, g_{h}((k-1) h)\right)$ for some $k>0$, that is, $\tau=(k-1) h$. Let $\varepsilon_{5}$ be the new wave, and let $\omega_{k-1}=\omega\left(A_{k-1}\right)$ be the change of angle.

If $\omega_{k-1} \leq 0$, then, by Lemma $4.2(2)$, we can choose suitable $K_{b}$ such that

$$
Q\left(U^{\nu, h} ; \tau+\right)-Q\left(U^{\nu, h} ; \tau-\right) \leq-\frac{K_{b}}{2}\left|\omega_{k}\right| L_{\mathrm{w}}\left(U^{\nu, h} ; \tau-\right) .
$$

In addition,

$$
L_{\mathrm{w}}\left(U^{\nu, h} ; \tau+\right)-L_{\mathrm{w}}\left(U^{\nu, h} ; \tau-\right)=0, \quad F_{0}\left(U^{\nu, h} ; \tau+\right) \leq F_{0}\left(U^{\nu, h} ; \tau-\right) .
$$

Hence, for $K>0$ and $C_{*}>0$, it follows that

$$
F\left(U^{\nu, h} ; \tau+\right)-F\left(U^{\nu, h} ; \tau-\right) \leq 0 .
$$


If $\omega_{k-1}>0$, then, by Lemma $4.2(\mathrm{i})$, for $L_{\mathrm{w}}\left(U^{\nu, h} ; \tau-\right)$ sufficiently small, we can choose suitable $K_{\mathrm{c}}$ such that

$$
Q\left(U^{\nu, h} ; \tau+\right)-Q\left(U^{\nu, h} ; \tau-\right) \leq-\frac{1}{2}\left|\omega_{k-1}\right|
$$

Moreover,

$$
L_{\mathrm{w}}\left(U^{\nu, h} ; \tau+\right)-L_{\mathrm{w}}\left(U^{\nu, h} ; \tau-\right)=O(1)\left|\omega_{k-1}\right|, \quad F_{1}\left(U^{\nu, h} ; \tau+\right)-F_{1}\left(U^{\nu, h} ; \tau-\right)=O(1)\left|\omega_{k-1}\right| .
$$

Thus we can choose $K$ and $C_{*}$ large enough such that

$$
F\left(U^{\nu, h} ; \tau+\right)-F\left(U^{\nu, h} ; \tau-\right) \leq-\frac{1}{4}\left|\omega_{k-1}\right| .
$$

With the estimates in Cases 1-3, we can choose positive constants $\delta_{2}, C_{*}, K_{0}, K_{1}, K_{2}$, and $K_{\mathrm{c}}$ independent of $(\tau, \nu)$ such that, if $F\left(U^{\nu, h} ; \tau-\right)<\delta_{2}$, then (6.5)-6.6) hold and, before $\tau_{1}$, the strength of every weak wave-front is less than $\delta_{*}$.

To prove statement (ii), we introduce the following functional for any strong rarefaction front $s_{0}(x)$ :

$$
F_{s}\left(U^{\nu, h} ; x\right)=\left|s_{0}(x)\right| e^{L_{s}\left(U^{\nu, h} ; x\right)+K_{2} Q\left(U^{\nu, h} ; x\right)},
$$

where

$$
L_{s}\left(U^{\nu, h} ; x\right)=\sum_{\alpha}\left\{|\alpha(x)|: \alpha \text { is a weak wave approaching } s_{0}\right\}
$$

and $K_{2}$ is a positive constant. Then we can carry out the steps as in [1, 4] to obtain (6.6).

Finally, we complete the proof of (iii). From estimate (6.5), we have known that the total interaction potential $Q\left(U^{\nu, h} ; x\right)$ is decreasing and bounded when it crosses an interaction time $x$. Then we conclude that, when the orders of the incoming waves are less than $\nu$ or the change of the angle of the boundary is larger than $\frac{1}{\nu}$, and $Q\left(U^{\nu, h} ; x\right)$ decreases by at least $-\frac{2^{-\nu}}{4}$ in these interactions. Therefore, we can follow the argument in [1, 4] to see that new physical waves can be only generated by this kind of interactions. When the weak wave $\alpha$ of 1-family interacts with the boundary and reflects, by solving the Riemann problem, we know that there is only a reflected wave of 5 -family with the reflected coefficient 1 , so that the number of the waves keeps the same, which implies that the number of the waves is finite between $(k-1) h<x<k h$. Since non-physical waves are generated only when physical waves interact, the number of non-physical waves is also finite. With the same procedure used in [1, 4, it can be proved that there exists a constant $C_{\mathrm{np}}>0$ such that (6.7) holds. This completes the proof.

To conclude the inductive hypothesis that $A_{2}(\tau-)$ holds and the total strength of the strong rarefaction is finite, we need the following proposition.

Proposition 6.2. For any $\tau<x<\tau_{1}$ and sufficiently small $\delta_{2}$ satisfying $0<\delta_{2}<\frac{\delta_{* *}}{4}$,

(i) There exists $\eta_{0}=\eta(x)>0$ such that, for $y>\eta_{0}$,

$$
\left|U^{\nu, h}(x, y)-U_{\infty}\right| \leq \delta_{2}
$$

(ii) $\left.U^{\nu, h}(x, \cdot)\right|_{x<\tau_{1}} \in D_{\delta_{* *}}\left(U_{\infty}\right)$; 
(iii) For any strong rarefaction wave-front $s_{0}(x)$,

$$
\sum_{s_{0}}\left|s_{0}(x)\right| \leq O(1)\left(\left|\theta_{\text {crit }}\right|+\delta_{0}+\delta_{2}+L_{\mathrm{w}}\left(U^{\nu, h} ; x\right)\right),
$$

where $\tau_{1}$ is the same as in Proposition 6.1.

Proof. It suffices to prove (i). By Proposition 6.1, we can choose $\eta_{0}^{*}=\eta^{*}(x)>0$ such that there is no strong wave-front for $y>\eta_{0}^{*}$. Then there exists $0<\varepsilon_{*}<\frac{\delta_{2}}{8}$ such that

$$
T V \cdot\left\{U^{\nu, h}(x, \cdot) ;\left[\eta_{0}^{*}, \infty\right)\right\} \leq O(1) \varepsilon_{*} .
$$

Then, by $\left(\mathbf{H}_{2}\right)$, we can choose a constant $0<\delta_{2 * *}<\frac{\delta_{2}}{2}$ such that, for $y>\eta_{0}^{*}$,

$$
\begin{aligned}
\left|U^{\nu, h}(x, y)-U_{\infty}\right| & \leq T V \cdot\left\{U^{\nu, h}(x, \cdot) ;\left[\eta_{0}^{*}, \infty\right)\right\}+\left|U^{\nu, h}(x, \infty)-U_{\infty}\right| \\
& \leq O(1) \varepsilon_{*}+\delta_{2 * *}<\delta_{2} .
\end{aligned}
$$

Facts (ii) and (iii) are the direct consequences of Lemma 6.16.2, Proposition 6.1, and (i). This completes the proof.

\section{BV Bounds For the ReActing SteP}

In this section, we study the BV stability of the approximate solutions $U^{\nu, h}$ for the reacting step. As in $\S 6$, we have proved that the solution $U^{\nu, h}(x, y)$ is $B V$-stable for $x<k h, k \in \mathbf{N}_{+}$, and the Glimm-type functional is decreasing and the total number of wave-fronts is finite for $(m-1) h<x<m h, m \leq k$. Now we study the uniform bound on the total variation of the approximate solutions $U^{\nu, h}(x, y)$ with respect to the mesh length $h$ on line $x=k h$. As a first step, we analyze the change of the wave strengths before and after the reaction when the mesh length $h$ is sufficiently small. Then we study the change of the Glimm-type functional for the reacting step to obtain the uniform bound on the total variation of the approximate solutions by employing the monotonicity of the Glimm-type functional that has been proved in $\S 6$.

7.1. Local estimates on the reacting step. We now study the change of the wave strengths at the reaction steps. Arguing as in the construction of the approximation solutions, we assume that there is no wave interaction on line $x=k h$. The analysis is divided into four cases.

Case 1. The change of $i$-weak wave $\tilde{\alpha}_{i}, 1 \leq i \leq 5$, after reaction (see Fig. 7.1). Let $\alpha_{i}$ be the $i$-weak incoming wave with order $k \leq \nu$ before reaction, which connects $\tilde{\alpha}_{i}$ to $\tilde{U}_{a}$ with

$$
\tilde{U}_{a}=\Phi_{i}\left(\tilde{\alpha}_{i} ; \tilde{U}_{b}\right) .
$$

Suppose that $\alpha_{i}, 1 \leq j \leq 5$, are the $j$-weak outgoing wave after reaction and related by

$$
U_{a}=\Phi\left(\alpha ; U_{b}\right) .
$$

Then, by the construction of the approximate solutions and Lemma 4.5, we have

$$
\Phi\left(\alpha ; \tilde{U}_{b}+O(1)\|\bar{Z}\|_{\infty} e^{-L k h} h\right)=\Phi_{i}\left(\tilde{\alpha}_{i} ; \tilde{U}_{b}\right)+O(1)\|\bar{Z}\|_{\infty} e^{-L k h} h .
$$




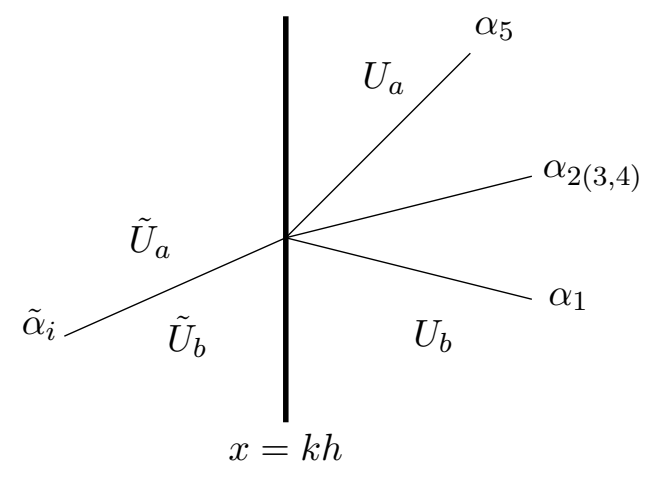

Fig. 7.1

Lemma 7.1. Equation (7.1) admits a unique $C^{2}-$ solution $\alpha=\alpha\left(\tilde{\alpha}_{i} ; e^{-L k h} h ; \tilde{U}_{b}\right)$ in a neighbourhood of $\left(\tilde{\alpha}_{i}, h\right)=(0,0)$. Moreover,

$$
\alpha_{i}=\tilde{\alpha}_{i}+O(1)\left|\tilde{\alpha}_{i}\right|\|\bar{Z}\|_{\infty} e^{-L k h} h, \quad \alpha_{j}=O(1)\left|\tilde{\alpha}_{i}\right|\|\bar{Z}\|_{\infty} e^{-L k h} h, \quad 1 \leq j \neq i \leq 5 .
$$

Proof. Notice that

$$
\begin{aligned}
& \left.\operatorname{det}\left(\frac{\partial \Phi\left(\alpha_{5}, \alpha_{4}, \alpha_{3}, \alpha_{2}, \alpha_{1} ; U_{b}\right)}{\partial\left(\alpha_{5}, \alpha_{4}, \alpha_{3}, \alpha_{2}, \alpha_{1}\right)}\right)\right|_{\alpha_{1}=\alpha_{2}=\alpha_{3}=\alpha_{4}=\alpha_{5}=h=0} \\
& =\operatorname{det}\left(\mathbf{r}_{5}, \mathbf{r}_{4}, \mathbf{r}_{3}, \mathbf{r}_{2}, \mathbf{r}_{1}\right)\left(\tilde{U}_{b}\right) \neq 0 .
\end{aligned}
$$

Then, by the implicit function theorem, there exists $\left(\alpha_{1}, \alpha_{2}, \alpha_{3}, \alpha_{4}, \alpha_{5}\right)$ as a $C^{2}$-function of $\left(\tilde{\alpha}_{i}, e^{-L k h} h\right)$ satisfying (17.1).

We now estimate $\alpha_{j}, 1 \leq j \leq 5$. For $j=i$, we have

$$
\alpha_{i}=\alpha_{i}\left(\tilde{\alpha}, 0 ; \tilde{U}_{b}\right)+\alpha_{i}\left(0,\|\bar{Z}\|_{\infty} e^{-L k h} h ; \tilde{U}_{b}\right)-\alpha_{i}\left(0,0 ; \tilde{U}_{b}\right)+O(1)|\tilde{\alpha}|\|\bar{Z}\|_{\infty} e^{-L k h} h .
$$

By (7.1), we have

$$
\alpha_{i}\left(\tilde{\alpha}, 0 ; \tilde{U}_{b}\right)=\tilde{\alpha}, \quad \alpha_{i}\left(0,\|\bar{Z}\|_{\infty} e^{-L k h} h ; \tilde{U}_{b}\right)=\alpha_{i}\left(0,0 ; \tilde{U}_{b}\right)=0 .
$$

Notice that

$$
\alpha_{j}\left(\tilde{\alpha}, 0 ; \tilde{U}_{b}\right)=\alpha_{j}\left(0,\|\bar{Z}\|_{\infty} e^{-L k h} h ; \tilde{U}_{b}\right)=\alpha_{j}\left(0,0 ; \tilde{U}_{b}\right)=0 .
$$

Then, in a similar way, we obtain the estimates for $\alpha_{j}, 1 \leq j \neq i \leq 5$. This completes the proof.

Case 2. The change of 5-strong rarefaction wave-fronts after reaction (see Fig. 7.2). Let $\tilde{s}$ be the 5 -strong incoming rarefaction fronts with order $k=1$ before reaction, which connects $\tilde{U}_{b}$ to $\tilde{U}_{a}$ with

$$
\tilde{U}_{a}=U_{\mathrm{Ra}}\left(\tilde{s} ; \tilde{U}_{b}\right) .
$$

Let $s$ be the 5 -strong rarefaction wave-front after reaction and related by

$$
U_{a}=U_{\mathrm{Ra}}\left(s ; \Phi\left(0, \alpha_{4}, \alpha_{3}, \alpha_{2}, \alpha_{1} ; U_{b}\right)\right) .
$$

Then, by the construction of the approximate solutions and Lemma 4.5, we have

$$
U_{\mathrm{Ra}}\left(s ; \Phi\left(0, \alpha_{2,3,4}, \alpha_{1} ; \tilde{U}_{l}+O(1)\|\bar{Z}\|_{\infty} e^{-L k h} h\right)\right)=U_{\operatorname{Ra}}\left(\tilde{s} ; \tilde{U}_{b}\right)+O(1)\|\bar{Z}\|_{\infty} e^{-L k h} h .
$$




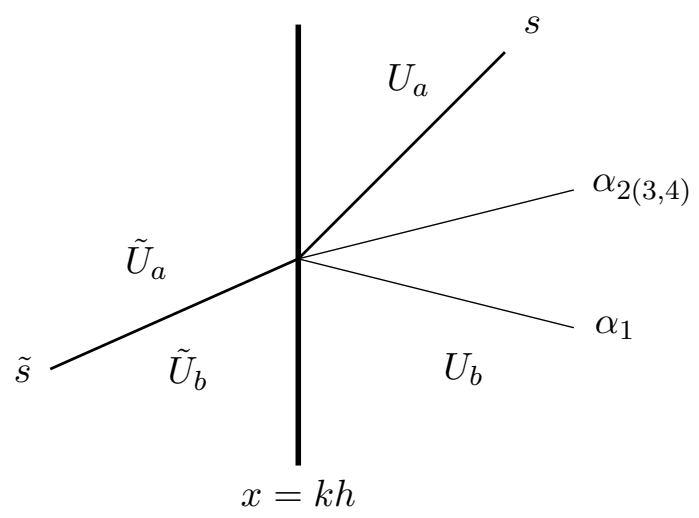

Fig. 7.2

Similar to Lemma 7.1, we obtain

Lemma 7.2. Let $\left\{\tilde{U}_{b}, \tilde{U}_{a}\right\}$ and $\left\{U_{b}, U_{a}\right\}$ be the constant states before and after reaction. Then

$$
\alpha_{i}=O(1)|\tilde{s}||| \bar{Z}\left\|_{\infty} e^{-L k h} h, i \neq 5, \quad s=\tilde{s}+O(1)|\tilde{s}|\right\| \bar{Z} \|_{\infty} e^{-L k h} h .
$$

Case 3. The change of non-physical wave-fronts $\epsilon$ after reaction (see Fig. 7.3). Let $\tilde{\epsilon}$ be the incoming non-physical wave-front with $\left\{\tilde{U}_{b}, \tilde{U}_{a}\right\}$ as its below and above states, respectively. Let $\epsilon$ be the outgoing non-physical wave-fronts after reaction and denote by $\left\{U_{b}, U_{a}\right\}$ as its below and above states, respectively.

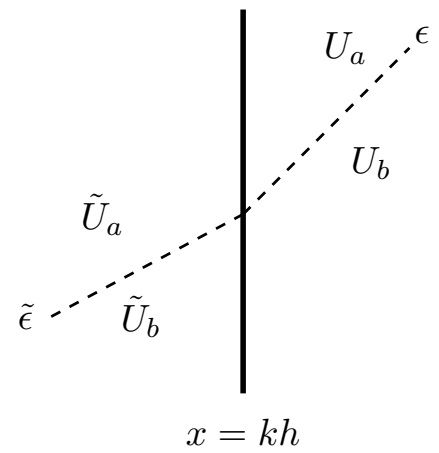

Fig. 7.3

Then we have the following lemma whose proof is similar to Lemma 7.1 .

Lemma 7.3. For $\tilde{\epsilon}$ and $\epsilon$ defined above, we have

$$
\epsilon=\tilde{\epsilon}+O(1)|\tilde{\epsilon}|\|\bar{Z}\|_{\infty} e^{-L k h} h .
$$

Case 4. New waves generated by the corner points after reaction (see Fig. 7.4). We consider the flow past a corner point $A_{k}=\left(k h, g_{h}(k h)\right)$ after reaction for some $k \in \mathbf{N}_{+}$. Denote by $\tilde{U}_{a}$ and $U_{a}$ the states before and after reaction. Let $\varepsilon$ be 5 -waves generated from $A_{k}$ after reaction. 


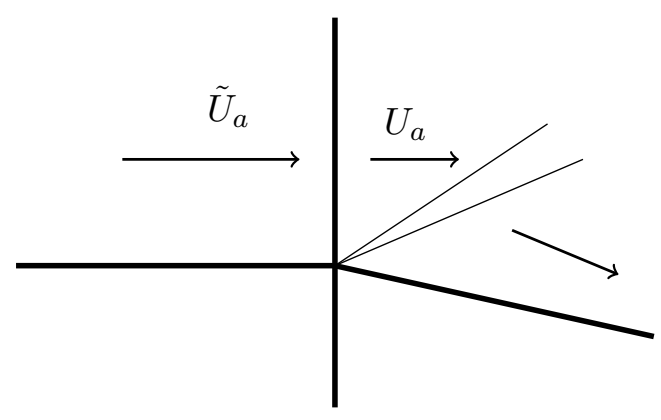

$x=k h$

Fig. 7.4

Then we have

$$
U_{a}=\tilde{U}_{a}+O(1)\|\bar{Z}\|_{\infty} e^{-L k h} h, \quad \Phi_{5}\left(\varepsilon ; U_{a}\right) \cdot\left(\mathbf{n}_{k}, 0,0,0\right)=0 .
$$

Using Lemmas 4.24.5 and by direct computation, we have

Lemma 7.4. Equation (7.6) admits a unique solution $\varepsilon$ such that

$$
\varepsilon=O(1) \omega_{k}+O(1)\|\bar{Z}\|_{\infty} e^{-L k h} h .
$$

7.2. Global estimates on the reacting step. In this subsection, we study the uniform bound on the total variation of the approximate solutions for the nonhomogeneous system (1.1). To do this, we first establish the relation of two functionals before and after reaction. To begin with, denote

$$
\omega_{k}^{+}=\max \left\{\omega_{k}, 0\right\} \quad \text { for } \omega_{k}=\omega_{k}\left(A_{k}\right) .
$$

Then we have

Lemma 7.5. For sufficiently small $h$ and sufficiently large $K_{\mathrm{c}}$ and $K$, there exists a constant $\hat{K}>0$ depending only on the system such that

$$
\begin{aligned}
& F_{0}\left(U^{\nu, h} ; k h+\right)-F_{0}\left(U^{\nu, h} ; k h-\right) \\
& \leq O(1)\|\bar{Z}\|_{\infty} e^{-L k h} h\left(L_{\mathrm{w}}\left(U^{\nu, h} ; k h-\right)+1\right)\left(L_{\mathrm{w}}\left(U^{\nu, h} ; k h-\right)+3\right)-\hat{K}\left|\omega_{k}^{+}\right| .
\end{aligned}
$$

Proof. The proof is based on Lemmas 7.1 7.4. First we have

$$
\begin{aligned}
& L_{\mathrm{w}}\left(U^{\nu, h} ; k h+\right)-L_{\mathrm{w}}\left(U^{\nu, h} ; k h-\right) \\
& =\sum_{1 \leq i \leq 5} L_{\mathrm{w}}^{i}\left(U^{\nu, h} ; k h+\right)+L_{\mathrm{np}}\left(U^{\nu, h} ; k h+\right)-\sum_{1 \leq i \leq 5} L_{\mathrm{w}}^{i}\left(U^{\nu, h} ; k h-\right)-L_{\mathrm{np}}\left(U^{\nu, h} ; k h-\right) \\
& =O(1)\|\bar{Z}\|_{\infty} e^{-L k h} h \sum_{1 \leq i \leq 5} \sum_{\alpha_{i}}\left|\alpha_{i}\right|+O(1)\|\bar{Z}\|_{\infty} e^{-L k h} h \sum_{\epsilon}|\epsilon|+O(1)\|\bar{Z}\|_{\infty} e^{-L k h} h \\
& \leq O(1)\|\bar{Z}\|_{\infty} e^{-L k h} h\left(L_{\mathrm{w}}\left(U^{\nu, h} ; k h-\right)+1\right)+O(1)\left|\omega_{k}^{+}\right|,
\end{aligned}
$$


STEADY SUPERSONIC EXOTHERMICALLY REACTING EULER FLOW PAST BENDING WALLS 35 and

$$
\begin{aligned}
& Q_{0}\left(U^{\nu, h} ; k h+\right)-Q_{0}\left(U^{\nu, h} ; k h+\right) \\
& =\sum_{\left(\alpha_{i}, \beta_{j}\right) \in \mathcal{A}}\left|\alpha_{i}\right|\left|\beta_{j}\right|-\sum_{\left(\tilde{\alpha}_{i}, \tilde{\beta}_{j}\right) \in \mathcal{A}}\left|\tilde{\alpha}_{i}\right|\left|\tilde{\beta}_{j}\right| \\
& =\sum_{i, j}\left(\left|\alpha_{i}\right|\left(\left|\beta_{j}\right|-\left|\tilde{\beta}_{j}\right|\right)+\left|\tilde{\beta}_{j}\right|\left(\left|\alpha_{i}\right|-\left|\tilde{\alpha}_{i}\right|\right)\right) \\
& \leq\left(L_{\mathrm{w}}\left(U^{\nu, h} ; k h+\right)-L_{\mathrm{w}}\left(U^{\nu, h} ; k h-\right)\right) L_{\mathrm{w}}\left(U^{\nu, h} ; k h-\right) \\
& \leq O(1)\|\bar{Z}\|_{\infty} e^{-L k h} h\left(L_{\mathrm{w}}\left(U^{\nu, h} ; k h-\right)+1\right) L_{\mathrm{w}}\left(U^{\nu, h} ; k h-\right) \\
& \leq O(1)\|\bar{Z}\|_{\infty} e^{-L k h} h\left(L_{\mathrm{w}}\left(U^{\nu, h} ; k h-\right)+1\right)^{2}+O(1)\left|\omega_{k}^{+}\right| .
\end{aligned}
$$

For $1 \leq i \leq 4$, we have

$$
\begin{aligned}
& Q_{B i}\left(U^{\nu, h} ; k h+\right)-Q_{B i}\left(U^{\nu, h} ; k h-\right) \\
& =\sum_{\alpha_{i}}\left|\alpha_{i}\right| W\left(\alpha_{i}, k h+,-\right)-\sum_{\tilde{\alpha}_{i}}\left|\tilde{\alpha}_{i}\right| W\left(\tilde{\alpha}_{i}, k h-,-\right) \\
& =\sum_{\tilde{\alpha}_{i}}\left|\tilde{\alpha}_{i}\right| W\left(\tilde{\alpha}_{i}, k h-,-\right)\left(\left(1+O(1)\|\bar{Z}\|_{\infty} e^{-L k h} h\right) \exp \left(O(1)\|\bar{Z}\|_{\infty} e^{-L k h} h\right)-1\right) \\
& \leq O(1)\|\bar{Z}\|_{\infty} e^{-L k h} h L_{\mathrm{w}}\left(U^{\nu, h} ; k h-\right) .
\end{aligned}
$$

Similarly,

$$
\begin{aligned}
& Q_{B 5}\left(U^{\nu, h} ; k h+\right)-Q_{B 5}\left(U^{\nu, h} ; k h-\right) \leq O(1)\|\bar{Z}\|_{\infty} e^{-L k h} h L_{\mathrm{w}}\left(U^{\nu, h} ; k h-\right)+O(1)\left|\omega_{k}^{+}\right|, \\
& Q_{B N P}\left(U^{\nu, h} ; k h+\right)-Q_{B N P}\left(U^{\nu, h} ; k h-\right) \\
& \quad=\sum_{\epsilon}|\epsilon| W(\epsilon, k h+,+)-\sum_{\tilde{\epsilon}}|\tilde{\epsilon}| W(\tilde{\epsilon}, k h-,+) \\
& \quad=\sum_{\tilde{\epsilon}}|\tilde{\epsilon}| W(\tilde{\epsilon}, k h-,+)\left(\left(1+O(1)\|\bar{Z}\|_{\infty} e^{-L k h} h\right) \exp \left(O(1)\|\bar{Z}\|_{\infty} e^{-L k h} h\right)-1\right) \\
& \quad \leq O(1)\|\bar{Z}\|_{\infty} e^{-L k h} h L_{\mathrm{w}}\left(U^{\nu, h} ; k h-\right), \\
& Q_{\mathrm{c}}\left(U^{\nu, h} ; k h+\right)-Q_{\mathrm{c}}\left(U^{\nu, h} ; k h-\right)=-\left|\omega_{k}^{+}\right| .
\end{aligned}
$$

Therefore, it follows that

$$
\begin{aligned}
& Q\left(U^{\nu, h} ; k h+\right)-Q\left(U^{\nu, h} ; k h-\right) \\
& \leq O(1)\|\bar{Z}\|_{\infty} e^{-L k h} h\left(L_{\mathrm{w}}\left(U^{\nu, h} ; k h-\right)+1\right)^{2}+O(1)\|\bar{Z}\|_{\infty} e^{-L k h} h L_{\mathrm{w}}\left(U^{\nu, h} ; k h-\right) \\
& \leq O(1)\|\bar{Z}\|_{\infty} e^{-L k h} h\left(L_{\mathrm{w}}\left(U^{\nu, h} ; k h-\right)+1\right)\left(L_{\mathrm{w}}\left(U^{\nu, h} ; k h-\right)+2\right)-\left(K_{\mathrm{c}}-O(1)\right)\left|\omega_{k}^{+}\right|,
\end{aligned}
$$


and

$$
\begin{aligned}
& F_{0}\left(U^{\nu, h} ; k h+\right)-F_{0}\left(U^{\nu, h} ; k h-\right) \\
&=L_{\mathrm{w}}\left(U^{\nu, h} ; k h+\right)-L_{\mathrm{w}}\left(U^{\nu, h} ; k h-\right)+K\left(Q\left(U^{\nu, h} ; k h+\right)-Q\left(U^{\nu, h} ; k h-\right)\right) \\
& \leq O(1)\|\bar{Z}\|_{\infty} e^{-L k h} h\left(L_{\mathrm{w}}\left(U^{\nu, h} ; k h-\right)+1\right) \\
&+O(1) e^{-L k h} h\left(L_{\mathrm{w}}\left(U^{\nu, h} ; k h+\right)+1\right)\left(L_{\mathrm{w}}\left(U^{\nu, h} ; k h+\right)+2\right) \\
&-K\left(K_{\mathrm{c}}-O(1)\right)\left|\omega_{k}^{+}\right|+O(1)\left|\omega_{k}^{+}\right| \\
& \leq O(1)\|\bar{Z}\|_{\infty} e^{-L k h} h\left(L_{\mathrm{w}}\left(U^{\nu, h} ; k h-\right)+1\right)\left(L_{\mathrm{w}}\left(U^{\nu, h} ; k h-\right)+3\right)-\hat{K}\left|\omega_{k}^{+}\right|,
\end{aligned}
$$

by choosing $K$ and $K_{\mathrm{c}}$ sufficiently large. This completes the proof.

To obtain the bound on the functional, we also need to estimate $F_{1}\left(U^{\nu, h} ; k h\right)$.

Lemma 7.6. For the approximate solutions $U^{\nu, h}$,

$$
\begin{aligned}
& F_{1}\left(U^{\nu, h} ; k h+\right)-F_{1}\left(U^{\nu, h} ; k h-\right) \\
& \leq O(1)\|\bar{Z}\|_{\infty} e^{-L k h} h\left(L_{\mathrm{w}}\left(U^{\nu, h} ; k h-\right)+1\right)+O(1)\left|\omega_{k}^{+}\right| .
\end{aligned}
$$

Proof. We complete the proof by combining Lemmas 7.17 .4 together. Here we only give a detail proof for Case 1, since the other cases can be treated in the same way.

Without loss of generality, consider a 1 -wave-front $\tilde{\alpha}_{1}$ that connects $\left\{\tilde{U}_{b}, \tilde{U}_{a}\right\}$ and intersects on line $x=k h$. Suppose that the new Riemann problem is solved by waves $\alpha_{i}, 1 \leq i \leq 5$, with $\left\{U_{b}, U_{m 1}, U_{m 2}, U_{a}\right\}$ as its below, middle, and above states, respectively (see Fig. 7.5).

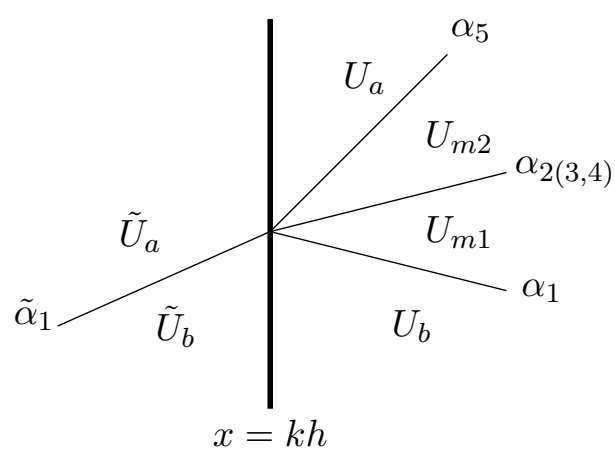

Fig. 7.5

\section{Denote}

$$
\theta(\Phi(\alpha ; U))=f\left(\alpha_{5}, \alpha_{2(3,4)}, \alpha_{1} ; U\right)
$$


STEADY SUPERSONIC EXOTHERMICALLY REACTING EULER FLOW PAST BENDING WALLS 37

Then, by Lemma 7.1, we have

$$
\begin{aligned}
&\left|\theta\left(U_{m 1}\right)-\theta\left(U_{b}\right)\right|-\left|\theta\left(\tilde{U}_{a}\right)-\theta\left(\tilde{U}_{b}\right)\right| \\
& \leq\left|\theta\left(U_{m 1}\right)-\theta\left(U_{b}\right)-\theta\left(\tilde{U}_{a}\right)+\theta\left(\tilde{U}_{b}\right)\right| \\
&=\left|f\left(0,0, \alpha_{1} ; U_{b}\right)-f\left(0,0,0 ; U_{b}\right)-f\left(0,0, \tilde{\alpha}_{1} ; \tilde{U}_{b}\right)+f\left(0,0,0 ; \tilde{U}_{b}\right)\right| \\
& \leq\left|f\left(0,0, \tilde{\alpha}_{1} ; \tilde{U}_{b}+O(1)\|\bar{Z}\|_{\infty} e^{-L k h} h\right)-f\left(0,0,0 ; \tilde{U}_{b}+O(1)\|\bar{Z}\|_{\infty} e^{-L k h} h\right)\right| \\
&+\left|f\left(0,0, \tilde{\alpha}_{1} ; \tilde{U}_{b}\right)-f\left(0,0,0 ; \tilde{U}_{b}\right)\right|+O(1)\|\bar{Z}\|_{\infty}\left|\tilde{\alpha}_{1}\right| e^{-L k h} h \\
&= O(1)\|\bar{Z}\|_{\infty}\left|\tilde{\alpha}_{1}\right| e^{-L k h} h,
\end{aligned}
$$

and

$$
\begin{aligned}
\theta\left(U_{a}\right)-\theta\left(U_{m 2}\right) & =f\left(\alpha_{5}, 0,0 ; U_{m 2}\right)-f\left(0,0,0 ; U_{m 2}\right) \\
& =f\left(O(1)\|\bar{Z}\|_{\infty}\left|\tilde{\alpha}_{1}\right| e^{-L k h} h, 0,0 ; U_{m 2}\right)-f\left(0,0,0 ; U_{m 2}\right) \\
& =O(1)\|\bar{Z}\|_{\infty}\left|\tilde{\alpha}_{1}\right| e^{-L k h} h .
\end{aligned}
$$

Since $\theta\left(U_{m 2}\right)=\theta\left(U_{m 1}\right)$, we have

$$
\begin{aligned}
& T V \cdot\left\{\theta\left(U^{\nu, h}(\tau+, \cdot)\right) ;\left[g_{h}(\tau+), \infty\right)\right\}-T V \cdot\left\{\theta\left(U^{\nu, h}(\tau-, \cdot)\right) ;\left[g_{h}(\tau-), \infty\right)\right\} \\
& =\sum_{\tilde{\alpha}_{1}}\left(\left|\theta\left(U_{a}\right)-\theta\left(U_{m 2}\right)\right|+\left|\theta\left(U_{m 2}\right)-\theta\left(U_{m 1}\right)\right|+\left|\theta\left(U_{m 1}\right)-\theta\left(U_{b}\right)\right|-\left|\theta\left(\tilde{U}_{a}\right)-\theta\left(\tilde{U}_{b}\right)\right|\right) \\
& =O(1)\|\bar{Z}\|_{\infty} \sum_{\tilde{\alpha}_{1}}\left|\tilde{\alpha}_{1}\right| e^{-L k h} h=O(1)\|\bar{Z}\|_{\infty} e^{-L k h} h L_{\mathrm{w}}\left(U^{\nu, h} ; k h-\right),
\end{aligned}
$$

which leads to

$$
F_{1}\left(U^{\nu, h} ; \tau+\right)-F_{1}\left(U^{\nu, h} ; \tau-\right)=O(1)\|\bar{Z}\|_{\infty} e^{-L k h} h L_{\mathrm{w}}\left(U^{\nu, h} ; k h-\right) .
$$

Similarly, by Lemma 6.1 and Proposition 6.2, we find that, for Cases 2-4,

$$
\begin{aligned}
F_{1}\left(U^{\nu, h} ; \tau+\right)-F_{1}\left(U^{\nu, h} ; \tau-\right) & =O(1)\|\bar{Z}\|_{\infty} \sum_{\tilde{s}}|\tilde{s}| e^{-L k h} h=O(1)\|\bar{Z}\|_{\infty} e^{-L k h} h, \\
F_{1}\left(U^{\nu, h} ; \tau+\right)-F_{1}\left(U^{\nu, h} ; \tau-\right) & =O(1)\|\bar{Z}\|_{\infty} \sum_{\tilde{\epsilon}}|\tilde{\epsilon}| e^{-L k h} h \\
& =O(1)\|\bar{Z}\|_{\infty} e^{-L k h} h L_{\mathrm{w}}\left(U^{\nu, h} ; k h-\right), \\
F_{1}\left(U^{\nu, h} ; \tau+\right)-F_{1}\left(U^{\nu, h} ; \tau-\right) & =O(1) \omega_{k}^{+}+O(1)\|\bar{Z}\|_{\infty} e^{-L k h} h .
\end{aligned}
$$

Finally, combining the above cases together, we conclude (7.9) .

With Lemma 7.5 7.6, we are now able to estimate on the change of functional $F\left(U^{\nu, h} ; x\right)$ at $x=k h$.

Lemma 7.7. For sufficiently small $h$, there exist $C_{4}$ independent of $(k, h)$ and $C_{*}$ sufficiently large such that, on line $x=k h$,

$$
F\left(U^{\nu, h} ; k h+\right) \leq F\left(U^{\nu, h} ; k h-\right)+C_{4}\|\bar{Z}\|_{\infty} e^{-L k h} h\left(F\left(U^{\nu, h} ; k h-\right)+4\right)^{2} .
$$


Proof. By Lemmas 7.5 7.6, we have

$$
\begin{aligned}
& F\left(U^{\nu, h} ; k h+\right)-F\left(U^{\nu, h} ; k h-\right) \\
& =F_{0}\left(U^{\nu, h} ; k h+\right)-F_{0}\left(U^{\nu, h} ; k h-\right)+C_{*}\left(F_{1}\left(U^{\nu, h} ; k h+\right)-F_{1}\left(U^{\nu, h} ; k h-\right)\right) \\
& \leq O(1)\left(1+C_{*}\right)\|\bar{Z}\|_{\infty} e^{-L k h} h\left(L_{\mathrm{w}}\left(U^{\nu, h} ; k h-\right)+1\right)+\left(O(1)-C_{*} \hat{K}\right)\left|\omega_{k}^{+}\right| \\
& \quad+O(1)\|\bar{Z}\|_{\infty} e^{-L k h} h\left(L_{\mathrm{w}}\left(U^{\nu, h} ; k h-\right)+1\right)\left(L_{\mathrm{w}}\left(U^{\nu, h} ; k h-\right)+3\right) .
\end{aligned}
$$

Then we choose $C_{*}$ and $\hat{K}$ sufficiently large so that there exists $C_{4}$ such that

$$
F\left(U^{\nu, h} ; k h+\right)-F\left(U^{\nu, h} ; k h-\right) \leq C_{4}\|\bar{Z}\|_{\infty} e^{-L k h} h\left(F\left(U^{\nu, h} ; k h-\right)+4\right)^{2} .
$$

To obtain the uniform bound on the total variation of the approximate solutions $U^{\nu, h}$, we introduce the following functional:

$$
\mathcal{F}\left(U^{\nu, h} ; \tau\right)=F\left(U^{\nu, h} ; \tau\right)+\mathcal{K} \sum_{k h>\tau}\|\bar{Z}\|_{\infty} e^{-L k h} h,
$$

where $\mathcal{K}>0$ is a unknown constant to be determined later.

Proposition 7.1. Suppose that $U^{\nu, h}(x, y)$ is the approximate solution for the initialboundary value problem (1.1) and (1.10)-(1.11). Then, for $h$ and $\delta_{2}$ sufficiently small, there exists a positive constant $\mathcal{K}$ independent of $\left(h, \nu, \delta_{2}\right)$ such that, if

$$
\mathcal{F}\left(U^{\nu, h} ; \tau\right) \leq \delta_{2}
$$

then

$$
\mathcal{F}\left(U^{h} ; \tau_{2}\right) \leq \mathcal{F}\left(U^{h} ; \tau_{1}\right) \quad \text { for any } \tau_{2} \geq \tau_{1} .
$$

Moreover, on $x=k h+$,

(i) $U^{\nu, h}(x, y) \in D_{\delta_{0}}\left(U_{\infty}\right)$;

(ii) There exist constants $C_{6}, C_{7}>0$ independent of $(\nu, h)$ such that

$$
|s| \leq \frac{C_{6}}{\nu}, \quad \sum_{s}|s| \leq C_{7},
$$

for any strong rarefaction front $s(x)$;

(iii) There exists a constant $C_{9}>0$ depending only on the system such that

$$
\sum_{\epsilon}|\epsilon| \leq \frac{C_{9}}{2^{\nu}}
$$

for any non-physical wave $\epsilon$ after reaction.

Proof. We divide the proof into two cases. Suppose that $\mathcal{F}\left(U^{h} ; \tau\right)<\delta_{2}$ for sufficiently small $\delta_{2}$.

1. If $(k-1) h \leq \tau_{1} \leq \tau_{2}<k h$, then, by Proposition 6.1, we have

$$
\mathcal{F}\left(U^{\nu, h} ; \tau_{2}\right)-\mathcal{F}\left(U^{h} ; \tau_{1}\right)=F\left(U^{\nu, h} ; \tau_{2}\right)-F\left(U^{\nu, h} ; \tau_{1}\right)-\mathcal{K}\left(\tau_{2}-\tau_{1}\right)\|\bar{Z}\|_{\infty} e^{-L k h} h \leq 0 .
$$


STEADY SUPERSONIC EXOTHERMICALLY REACTING EULER FLOW PAST BENDING WALLS 39

2. If $(k-1) h \leq \tau_{1}<k h<\tau_{2}<(k+1) h$, then, by Proposition 6.1 and Lemma 7.7, we can choose $\mathcal{K}$ suitably large such that

$$
\begin{aligned}
\mathcal{F}\left(U^{\nu, h} ; \tau_{2}\right)-\mathcal{F}\left(U^{h} ; \tau_{1}\right) & =F\left(U^{\nu, h} ; \tau_{2}\right)-F\left(U^{\nu, h} ; \tau_{1}\right)-\mathcal{K}\left(\tau_{2}-\tau_{1}\right)\|\bar{Z}\|_{\infty} e^{-L k h} h \\
& \leq F\left(U^{\nu, h} ; k h+\right)-F\left(U^{\nu, h} ; k h-\right)-\mathcal{K}\left(\tau_{2}-\tau_{1}\right)\|\bar{Z}\|_{\infty} e^{-L k h} h \\
& \leq\left(C_{4}\left(\delta_{2}+4\right)^{2}-\mathcal{K}\left(\tau_{2}-\tau_{1}\right)\right)\|\bar{Z}\|_{\infty} e^{-L k h} h \leq 0 .
\end{aligned}
$$

Since $\bar{Z}(\infty)=0$, then $U^{\nu, h}(k h+, \infty)=U^{\nu, h}(k h-, \infty)=\bar{U}(\infty)$. On the other hand, by $\left(\mathbf{H}_{\mathbf{2}}\right)$, we can choose suitably $\delta_{2}$ such that

$$
\left\|\bar{U}-U_{\infty}\right\|_{\infty}<\delta_{2} .
$$

Therefore, by Lemma 6.2 and Proposition 17.1, for $\delta_{2}$ sufficiently small, we have

$$
\begin{aligned}
& \left|\left(J\left(q, \frac{q^{2}}{2}+\frac{c^{2}}{\gamma-1}\right)+\theta\right)\left(U^{\nu, h}(k h+, y)\right)-J\left(q_{\infty}, \frac{q_{\infty}^{2}}{2}+\frac{c_{\infty}^{2}}{\gamma-1}\right)\right| \\
& \leq\left|\left(J\left(q, \frac{q^{2}}{2}+\frac{c^{2}}{\gamma-1}\right)+\theta\right)\left(U^{\nu, h}(k h+, y)\right)-\left(J\left(q, \frac{q^{2}}{2}+\frac{c^{2}}{\gamma-1}\right)+\theta\right)\left(U^{\nu, h}(k h+, \infty)\right)\right| \\
& \quad+\left|\left(J\left(q, \frac{q^{2}}{2}+\frac{c^{2}}{\gamma-1}\right)+\theta\right)\left(U^{\nu, h}(k h+, \infty)\right)-J\left(q_{\infty}, \frac{q_{\infty}^{2}}{2}+\frac{c_{\infty}^{2}}{\gamma-1}\right)\right| \\
& \leq O(1) \mathcal{F}\left(U^{\nu, h} ; k h+\right)+\left|\left(J\left(q, \frac{q^{2}}{2}+\frac{c^{2}}{\gamma-1}\right)+\theta\right)(\bar{U}(\infty))-J\left(q_{\infty}, \frac{q_{\infty}^{2}}{2}+\frac{c_{\infty}^{2}}{\gamma-1}\right)\right| \leq \delta_{0} .
\end{aligned}
$$

Similarly,

$$
\begin{aligned}
& \left|\left(\frac{q^{2}}{2}+\frac{c^{2}}{\gamma-1}\right)\left(U^{\nu, h}(k h+, y)\right)-\frac{q_{\infty}^{2}}{2}-\frac{c_{\infty}^{2}}{\gamma-1}\right| \leq \delta_{0}, \\
& \left|\frac{p^{\nu, h}}{\left(\rho^{\nu, h}\right)^{\gamma}}(k h+, y)-\frac{p_{\infty}}{\rho_{\infty}^{\gamma}}\right| \leq \delta_{0}, \quad 0 \leq Z^{\nu, h}(k h+, y) \leq \delta_{0} .
\end{aligned}
$$

Notice that

$$
\theta\left(U^{\nu, h}(k h+; y)\right)=\left(\theta\left(U^{\nu, h}(k h+; y)\right)-\theta\left(U^{\nu, h}(k h+, \infty)\right)\right)+\left(\theta\left(U^{\nu, h}(k h+, \infty)\right)-\theta\left(U_{\infty}\right)\right) .
$$

Then, by Lemma 6.1 and Proposition 17.1, for sufficiently small $\delta_{2}$, we have

$$
\theta\left(U^{\nu, h}(k h+; y)\right) \leq O(1) \mathcal{F}\left(U^{\nu, h} ; k h+\right)+O(1) \delta_{2} \leq \delta_{0} .
$$

On the other hand, we have

$$
\begin{aligned}
\theta\left(U^{\nu, h}(k h+; y)\right) & =\left(\theta\left(U^{\nu, h}(k h+; y)\right)-\theta\left(U^{\nu, h}(k h+, \infty)\right)\right)+\left(\theta\left(U^{\nu, h}(k h+, \infty)\right)-\theta\left(U_{\infty}\right)\right) \\
& \geq-T V \cdot\left\{\theta\left(U^{\nu, h}(k h+,, y)\right)\right\}+\theta_{\text {crit }}+O(1) \delta_{2} \\
& \geq-F\left(U^{h} ; k h+\right)+\theta_{\text {crit }}+O(1) \delta_{2} \geq \theta_{\text {crit }}+\delta_{0} .
\end{aligned}
$$

Finally, by Lemma 7.2, there exists a constant $C_{5}>0$ such that

$$
\begin{aligned}
|s| & \leq\left(1+O(1)\|\bar{Z}\|_{\infty} e^{-L k h} h\right)|\tilde{s}| \leq\left(1+O(1)\|\bar{Z}\|_{\infty} e^{-L k h} h\right) \frac{C_{3}}{\nu} \\
& \leq \frac{C_{3} \exp \left(C_{5} / L\right)}{\nu} .
\end{aligned}
$$


Then we can find a constant $C_{6}>C_{3} \exp \left(C_{5} / L\right)$ such that

$$
|s| \leq \frac{C_{6}}{\nu}
$$

Finally, by Proposition 6.2 and Lemma 7.2 , we can find $C_{7}>C_{4} \exp \left(C_{5} / L\right)$ such that

$$
\sum|s| \leq\left(1+O(1)\|\bar{Z}\|_{\infty} e^{-L k h} h\right) \sum|\tilde{s}| \leq \exp \left(C_{5} \int_{0}^{\infty} e^{-x} d x\right) \sum|\tilde{s}| \leq C_{7} .
$$

Also, by Proposition 6.1 and Lemma 7.3, we can find positive constants $C_{8}$ and $C_{9}$ such that

$$
\begin{aligned}
& \sum|\epsilon| \leq\left(1+O(1)\|\bar{Z}\|_{\infty} e^{-L k h} h\right) \sum|\tilde{\epsilon}| \leq\left(1+C_{8}\|\bar{Z}\|_{\infty} e^{-L k h} h\right) \sum|\tilde{\epsilon}| \\
& \leq \exp \left(C_{\mathrm{np}} \int_{0}^{\infty} e^{-x} d x\right) \sum|\tilde{\epsilon}| \leq \frac{C_{9}}{2^{\nu}} .
\end{aligned}
$$

This completes the proof.

Corollary 7.1. There exist positive constants $\varepsilon$ and $C_{0}$ independent of $(\nu, h)$ such that, if

$$
\left\|\bar{U}-U_{\infty}\right\|_{\infty}+\left|g_{+}^{\prime}(0)-g_{*+}^{\prime}(0)\right|+T V \cdot\{\bar{U}(\cdot)\}+T V \cdot\left\{\left(g_{+}^{\prime}-g_{*+}^{\prime}\right)(\cdot)\right\}<\varepsilon,
$$

then

$$
T V \cdot\left\{U^{\nu, h}(x, \cdot)\right\}<C_{0} .
$$

Proof. By Proposition 7.1, if $\mathcal{F}\left(U^{\nu, h} ; 0+\right)$ sufficiently small, we deduce

$$
\mathcal{F}\left(U^{\nu, h} ; x\right)<\mathcal{F}\left(U^{\nu, h} ; 0+\right)
$$

Since

$$
\begin{aligned}
\mathcal{F}\left(U^{\nu, h} ; 0+\right) & =F\left(U^{\nu, h} ; 0+\right)+C_{4}\|\bar{Z}\|_{\infty} \sum_{l=0}^{l=k} e^{-L k h} h \\
& \leq F\left(U^{\nu, h} ; 0+\right)+C_{4}\|\bar{Z}\|_{\infty} \int_{0}^{\infty} e^{-\tau} d \tau \\
& \leq F\left(U^{\nu, h} ; 0+\right)+\frac{C_{4}}{L}\|\bar{Z}\|_{\infty} \\
& \leq O(1) F\left(U^{\nu, h} ; 0+\right),
\end{aligned}
$$

where we have used that $\bar{Z}(\infty)=0$. On the other hand,

$$
\begin{aligned}
& F\left(U^{\nu, h} ; 0+\right)=O(1)\left(\left\|\bar{U}-U_{\infty}\right\|_{\infty}+T V \cdot\{\bar{U}(\cdot)\}+\sum_{k \geq 0}\left|\omega_{k}^{+}\right|\right), \\
& \sum_{k \geq 0}\left|\omega_{k}^{+}\right|=O(1)\left(T V \cdot\left\{\left(g_{+}^{\prime}-g_{*+}^{\prime}\right)(\cdot)\right\}+\left|g_{+}^{\prime}(0)-g_{*+}^{\prime}(0)\right|\right) .
\end{aligned}
$$

Thus, combining the above estimates, we obtain the desire result. 
STEADY SUPERSONIC EXOTHERMICALLY REACTING EULER FLOW PAST BENDING WALLS 41

\section{Convergence And Consistency}

In this section, we show that there exists a subsequence of the approximate solutions which converges to an entropy solution to the initial-boundary value problem (1.10)-(1.11) for system (1.1). To see this, we proceed by two steps:

(a) We establish the consistency of our algorithm by keeping $h>0$ fixed and letting $\nu \rightarrow \infty$.

(b) We employ the compactness theorem to show the convergence of a subsequence of the approximate solutions as $h \rightarrow 0$.

First, if line $x=a>0$ intersects with $\Gamma_{h}$ at point $\left(a, g_{h}(a)\right)$, we have

Lemma 8.1. For any $x^{\prime}, x^{\prime \prime} \in((k-1) h, k h), k \in \mathbb{N}_{+}$, there exists a constant $M_{0}>0$ independent of $(\nu, h)$ such that

$$
\int_{0}^{\infty}\left|U^{\nu, h}\left(x^{\prime}, y+g_{h}\left(x^{\prime}\right)\right)-U^{\nu, h}\left(x^{\prime \prime}, y+g_{h}\left(x^{\prime \prime}\right)\right)\right| d y \leq M_{0}\left|x^{\prime}-x^{\prime \prime}\right| .
$$

The proof directly follows from the construction of the approximate solutions $U^{\nu, h}$ and the bound on its total variation, which leads to the uniform Lipschitz continuity.

Proposition 8.1. Let $U^{\nu, h}$ be a family of the approximate solutions of problem (1.10)(1.11) for system (1.1). Then there exists a subsequence $\left\{\nu_{i}\right\}_{i=1}^{\infty}$ such that $U^{\nu_{i}, h}$ converges to a function $U^{h}$ in $\mathrm{L}_{\text {loc }}^{1}\left(\Omega_{h}\right)$ as $i \rightarrow \infty$, and the limit function $U^{h}(x, y)$ satisfies that, for any $\psi \in C_{0}^{\infty}\left(\Omega_{h}\right)$ with $\psi \geq 0$ and for any convex entropy pair $(\eta, q)$,

$$
\begin{array}{r}
\iint_{\Omega_{h}}\left(\eta\left(W\left(U^{h}\right)\right) \psi_{x}+q\left(W\left(U^{h}\right)\right) \psi_{y}\right) d x d y+\int_{y>0} \eta(W(\bar{U}(y))) \psi(0, y) d y \\
+\sum_{k \geq 1} h \int_{y>g_{k}} \nabla_{W} \eta\left(W\left(U^{h}(k h-, y)\right)\right) G\left(U^{h}(k h-, y)\right) \psi(k h, y) d y \\
+\int_{y>0} \eta(W(\bar{U}(y))) \psi(0, y) d y+\sum_{k \geq 1} h \int_{y>g_{k}} \mathcal{O}^{\eta}(h, k h-, y) \psi(k h, y) d y \geq 0
\end{array}
$$

where $\mathcal{O}^{\eta}(h, k h-, y) \rightarrow 0$ as $h \rightarrow 0$, and $\mathcal{O}^{\eta}(h, k h-, y) \equiv 0$ when $\eta= \pm W$.

Proof. We first denote

$$
\begin{aligned}
\mathcal{N}^{\nu, h}(\psi):= & \iint_{\Omega_{h}}\left(\eta\left(W\left(U^{\nu, h}\right)\right) \psi_{x}+q\left(W\left(U^{\nu, h}\right)\right) \psi_{y}\right) d x d y+\int_{y>0} \eta\left(W\left(\bar{U}^{\nu}(y)\right)\right) \psi(0, y) d y \\
& +\sum_{k \geq 1} h \int_{y>g_{k}} \nabla_{W} \eta\left(W\left(U^{\nu, h}(k h-, y)\right)\right) G\left(U^{\nu, h}(k h-, y)\right) \psi(k h, y) d y \\
& +\sum_{k \geq 1} h \int_{y>g_{k}} \mathcal{O}_{\nu}^{\eta}(h, k h, y) \psi(k h, y) d y \\
= & : \mathcal{N}_{1}^{\nu}(\psi)+\mathcal{N}_{2}^{\nu}(\psi)
\end{aligned}
$$


where

$$
\begin{aligned}
\mathcal{N}_{1}^{\nu}(\psi)= & \iint_{\Omega_{h}}\left(\eta\left(W\left(U^{\nu, h}\right)\right) \psi_{x}+q\left(W\left(U^{\nu, h}\right)\right) \psi_{y}\right) d x d y+\int_{y>0} \eta\left(W\left(\bar{U}^{\nu}(y)\right)\right) \psi(0, y) d y, \\
\mathcal{N}_{2}^{\nu}(\psi)= & \sum_{k \geq 1} h \int_{y>g_{k}} \nabla_{W} \eta\left(W\left(U^{\nu, h}(k h-, y)\right)\right) G\left(U^{\nu, h}(k h-, y)\right) \psi(k h, y) d y \\
& +\sum_{k \geq 1} h \int_{y>g_{k}} \mathcal{O}_{\nu}^{\eta}(h, k h, y) \psi(k h, y) d y .
\end{aligned}
$$

Let $\Xi(x, y)=\left(\eta\left(W\left(U^{\nu, h}\right)\right) \psi, q\left(W\left(U^{\nu, h}\right)\right) \psi\right)(x, y)$. Then, by the divergence theorem, we have

$$
\begin{aligned}
\mathcal{N}_{1}^{\nu, h}(\phi)= & \sum_{k} \iint_{\Omega_{h, k}}\left(\eta\left(W\left(U^{\nu, h}\right)\right) \psi_{x}+q\left(W\left(U^{\nu, h}\right)\right) \psi_{y}\right) d x d y+\int_{y>0} \eta\left(W\left(\bar{U}^{\nu}(y)\right)\right) \psi(0, y) d y \\
= & \sum_{k, i} \int_{\Gamma_{h, k}^{i}} \operatorname{div} \Xi(x, y) \cdot \mathbf{n}_{k, i} d S-\sum_{k} \iint_{\Omega_{h, k}}\left(\eta\left(W\left(U^{\nu, h}\right)\right)_{x}+q\left(W\left(U^{\nu, h}\right)\right)_{y}\right) \psi d x d y \\
& +\int_{y>0} \eta(W(\bar{U}(y))) \psi(0, y) d y .
\end{aligned}
$$

Since $U^{\nu, h}$ is an entropy solution for the homogeneous system $W(U)_{x}+H(U)_{y}=0$ in $\Omega_{h, k}$, then

$$
\begin{aligned}
\mathcal{N}_{1}^{\nu, h}(\phi) \geq & \sum_{k} \int_{k h}^{(k+1) h} \sum_{\alpha \in \mathcal{J}} \mathcal{E}_{\nu, \alpha}(x) \psi\left(x, y_{\alpha}(x)\right) d x \\
& -\sum_{k \geq 1} \int_{y>g_{k}}\left(\eta \left(W\left(U^{\nu, h}(k h+, y)\right)-\eta\left(W\left(U^{\nu, h}(k h-, y)\right)\right) \psi(k h, y) d y,\right.\right.
\end{aligned}
$$

where

$$
\mathcal{E}_{\nu, \alpha}(x)=\dot{y}_{\alpha}(x)\left[\eta\left(W\left(U^{\nu, h}\left(x, y_{\alpha}(x)\right)\right)\right)\right]-\left[q\left(W\left(U^{\nu, h}\left(x, y_{\alpha}(x)\right)\right)\right)\right]
$$

with $\left[f\left(U^{\nu, h}\left(x, y_{\alpha}(x)\right)\right)\right]:=f\left(U^{\nu, h}\left(x, y_{\alpha}(x)+\right)\right)-f\left(U^{\nu, h}\left(x, y_{\alpha}(x)-\right)\right)$ for any function $f$.

Using the properties of the $(\nu, h)$-approximate solutions, then we have

$$
\sum_{\alpha \in \mathcal{S} \cup \mathcal{R} \cup \mathcal{R}_{b}} \mathcal{E}_{\nu, \alpha}(x) \geq-\sum_{\alpha \in \mathcal{S} \cup \mathcal{R} \cup \mathcal{R}_{b}}\left|\sigma_{\alpha}\right| \frac{O(1)}{2^{\nu}}, \quad \sum_{\alpha \in \mathcal{N} \mathcal{P}} \mathcal{E}_{\nu, \alpha}(x)=-O(1) \sum_{\alpha \in \mathcal{N} \mathcal{P}}\left|\sigma_{\alpha}\right|=\frac{O(1)}{2^{\nu}} .
$$

Thus it follows that

$$
\sum_{\alpha \in \mathcal{J}} \mathcal{E}_{\nu, \alpha}(x)=\sum_{\alpha \in \mathcal{S} \cup \mathcal{R} \cup \mathcal{R}_{b}} \mathcal{E}_{\nu, \alpha}(x)+\sum_{\alpha \in \mathcal{N} \mathcal{P}} \mathcal{E}_{\nu, \alpha}(x) \geq-\frac{O(1)}{2^{\nu}} .
$$

On the other hand, by the Talyor formula, we also find

$$
\begin{aligned}
& \eta\left(W\left(U^{\nu, h}(k h+, y)\right)\right)-\eta\left(W\left(U^{\nu, h}(k h-, y)\right)\right) \\
& =\nabla_{W} \eta\left(W\left(U^{\nu, h}(k h-, y)\right)\right) G\left(U^{\nu, h}(k h-, y)\right) h+\mathcal{O}_{\nu}^{\eta}(h, k h-, y) h,
\end{aligned}
$$


STEADY SUPERSONIC EXOTHERMICALLY REACTING EULER FLOW PAST BENDING WALLS 43

where $\mathcal{O}_{\nu}^{\eta}(h, k h-, y) \rightarrow 0$ as $h \rightarrow 0$, and $\mathcal{O}_{\nu}^{\eta}(h, k h-, y) \equiv 0$ when $\eta= \pm W$.

Finally, combining these estimates altogether, we deduce

$$
\mathcal{N}_{1}^{\nu}(\phi)+\mathcal{N}_{2}^{\nu}(\phi) \geq-\frac{O(1)}{2^{\nu}}
$$

Since the total variation of $U^{\nu, h}$ is uniformly bounded, then, by Lemma 8.1 and Helly's theorem, we can choose a subsequence $U^{\nu_{i}}$ such that

$$
U^{\nu_{i}, h}(x, y) \rightarrow U^{h}(x, y), \quad U^{\nu_{i}, h}(k h-, y) \rightarrow U^{h}(k h, y) \quad \text { a.e. in } \Omega_{h} \text { as } i \rightarrow \infty,
$$

and define $\mathcal{O}^{\eta}(h, k h-, y)=\lim _{\nu_{i} \rightarrow \infty} \mathcal{O}_{\nu_{i}}^{\eta}(h, k h-, y)$, which leads to the desired result.

Now we are in position to establish an existence theorem for the initial-boundary value problem (1.1) and (1.10)-(1.11). We can apply again the Helly compactness theorem to obtain a further subsequence $U^{h_{i}}$ converging to some function $U$ in $\mathbf{L}_{l o c}^{1}$ whose total variation in $y$ is uniformly bounded for all $x \geq 0$. Then we have the following proposition.

Proposition 8.2. Let $U^{h}$ be a sequence of approximate solutions determined by (8.2) with uniformly bounded total variation. Then there exists a subsequence $h_{i}$ such that $U^{h_{i}} \rightarrow U$ in $\mathbf{L}_{l o c}^{1}$ as $h_{i} \rightarrow 0$, and $U$ is a weak solution to the initial-boundary value problem (1.1) and (1.10) -(1.11) satisfying the entropy inequality: For any convex entropy pair $(\eta, q)$,

$$
\begin{aligned}
\iint_{\Omega}\left(\eta(W(U)) \psi_{x}+q(W(U)) \psi_{y}\right) d x d y & +\iint_{\Omega} \nabla_{W} \eta(W(U)) G(U) \psi d x d y \\
& +\int_{y>0} \eta(W(\bar{U}(y))) \psi(0, y) d y \geq 0
\end{aligned}
$$

for any $\psi \in C_{0}^{\infty}(\Omega)$ with $\psi \geq 0$. 
Proof. For any $\psi \in C_{0}^{\infty}\left(\Omega_{h}\right)$ with $\psi \geq 0$, we have

$$
\begin{aligned}
& \iint_{\Omega_{h}}\left(\eta\left(W\left(U^{h}\right)\right) \psi_{x}+q\left(W\left(U^{h}\right)\right) \psi_{y}\right) d x d y+\iint_{\Omega_{h}} \nabla_{W} \eta\left(W\left(U^{h}\right)\right) G\left(U^{h}\right) \psi d x d y \\
& \quad+\int_{y>0} \eta(W(\bar{U}(y))) \psi(0, y) d y+\sum_{k \geq 1} h \int_{y>g_{k}} \mathcal{O}^{\eta}(h, k h-, y) \psi(k h, y) d y \\
& \geq \iint_{\Omega_{h}} \nabla_{W} \eta\left(W\left(U^{h}\right)\right) G\left(U^{h}\right) \psi d x d y \\
& \quad-\sum_{k \geq 1} h \int_{y>g_{k}} \nabla_{W} \eta\left(W\left(U^{h}(k h-, y)\right)\right) G\left(U^{h}(k h-, y)\right) \psi(k h, y) d y \\
& =\sum_{k \geq 0} \int_{k h}^{(k+1) h} \int_{g_{h}(x)}^{\infty}\left(\nabla_{W} \eta\left(W\left(U^{h}\right)\right) G\left(U^{h}\right) \psi\right. \\
& \quad+\sum_{k \geq 0} \int_{k h}^{(k+1) h} \int_{g_{k}}^{g_{h}(x)} \nabla_{W} \eta\left(W\left(U^{h}(k h-, y)\right) G\left(U^{h}(k h-, y)\right) \psi(k h, y) d y d x\right. \\
& \quad-h \int_{0}^{\infty} \nabla_{W} \eta\left(W\left(U^{h}(0, y)\right) G\left(U^{h}(0, y)\right) \psi(0, y) d y\right. \\
& =: \Sigma_{1}+\Sigma_{2}-\Sigma_{3} .
\end{aligned}
$$

For $\Sigma_{2}-\Sigma_{3}$, we have

$$
\begin{aligned}
\left|\Sigma_{2}-\Sigma_{3}\right| \leq & \sum_{k \geq 0}\left|\int_{k h}^{(k+1) h} \int_{g_{k}}^{g_{h}(x)}\right| \nabla_{W} \eta\left(W\left(U^{h}(k h-, y)\right)\right)|| G\left(U^{h}(k h-, y)\right)|| \psi(k h, y)|d y d x| \\
& +h \int_{0}^{\infty}\left|\nabla_{W} \eta\left(W\left(U^{h}(0, y)\right)\right)\right|\left|G\left(U^{h}(0, y)\right)\right||\psi(0, y)| d y \\
\leq & O(1)\left(\frac{\operatorname{diam}(\operatorname{supp} \psi)}{h}\right)\left|g_{h}(x)-g_{k}\right| h+O(1) h \\
\leq & O(1)\left(\left|\tan \left(\theta_{\text {crit }}\right)\right|+1\right) h \rightarrow 0 \quad \text { as } h \rightarrow 0 .
\end{aligned}
$$

Now we estimate $\Sigma_{1}$ in (8.5). By direct computation, we have

$$
\begin{aligned}
\Sigma_{1}= & \sum_{k \geq 0} \int_{k h}^{(k+1) h} \int_{0}^{\infty}\left(\nabla_{W} \eta\left(W\left(U^{h}\left(x, y+g_{h}(x)\right)\right)\right) G\left(U^{h}\left(x, y+g_{h}(x)\right)\right) \psi\left(x, y+g_{h}(x)\right)\right. \\
& \left.-\nabla_{W} \eta\left(W\left(U^{h}\left(k h-, y+g_{h}(x)\right)\right)\right) G\left(U^{h}\left(k h-, y+g_{h}(x)\right)\right) \psi\left(k h, y+g_{h}(x)\right)\right) d y d x \\
= & \Sigma_{1}^{1}+\Sigma_{1}^{2}
\end{aligned}
$$


STEADY SUPERSONIC EXOTHERMICALLY REACTING EULER FLOW PAST BENDING WALLS 45

where

$$
\begin{aligned}
& \Sigma_{1}^{1}=\sum_{k \geq 0} \int_{k h}^{(k+1) h} \int_{0}^{\infty}\left(\nabla_{W} \eta\left(W\left(U^{h}\left(x, y+g_{h}(x)\right)\right)\right)-\nabla_{W} \eta\left(W\left(U^{h}\left(k h-, y+g_{h}(x)\right)\right)\right)\right) \\
& \times G\left(U^{h}\left(x, y+g_{h}(x)\right)\right) \psi\left(k h, y+g_{h}(x)\right) d y d x, \\
& \Sigma_{1}^{2}=\sum_{k \geq 0} \int_{k h}^{(k+1) h} \int_{0}^{\infty} \nabla_{W} \eta\left(W\left(U^{h}\left(k h-, y+g_{h}(x)\right)\right)\right) \\
& \times\left(G\left(U^{h}\left(k h+, y+g_{h}(x)\right)\right) \psi\left(x, y+g_{h}(x)\right)\right. \\
&\left.\quad-G\left(U^{h}\left(k h-, y+g_{h}(x)\right)\right) \psi\left(k h, y+g_{h}(x)\right)\right) d y d x .
\end{aligned}
$$

Now we consider $\Sigma_{1}^{2}$. By direct computation, we have

$$
\Sigma_{1}^{2}=I_{1}+I_{2}+I_{3}
$$

where

$$
\begin{aligned}
& I_{1}=\sum_{k \geq 0} \int_{k h}^{(k+1) h} \int_{0}^{\infty} \nabla_{W} \eta\left(W\left(U^{h}\left(k h-, y+g_{h}(x)\right)\right)\right) \\
& \quad \times\left(G\left(U^{h}\left(x, y+g_{h}(x)\right)\right)-G\left(U^{h}\left(k h+, y+g_{h}(x)\right)\right)\right) \psi\left(k h, y+g_{h}(x)\right) d y d x \\
& I_{2}=\sum_{k \geq 0} \int_{k h}^{(k+1) h} \int_{0}^{\infty} \nabla_{W} \eta\left(W\left(U^{h}\left(k h-, y+g_{h}(x)\right)\right)\right) \\
& \quad \times\left(G\left(U^{h}\left(k h+, y+g_{h}(x)\right)\right)-G\left(U^{h}\left(k h-, y+g_{h}(x)\right)\right)\right) \psi\left(k h, y+g_{h}(x)\right) d y d x \\
& I_{3}=\sum_{k \geq 0} \int_{k h}^{(k+1) h} \int_{0}^{\infty} \nabla_{W} \eta\left(W\left(U^{h}\left(k h-, y+g_{h}(x)\right)\right)\right) \\
& \quad \times\left(\psi\left(x, y+g_{h}(x)\right)-\psi\left(k h, y+g_{h}(x)\right)\right) G\left(U^{h}\left(x, y+g_{h}(x)\right)\right) d y d x .
\end{aligned}
$$

For $I_{1}$, in view of Lemma 8.1, we have

$$
\begin{aligned}
\left|I_{1}\right| \leq O(1) \sum_{0 \leq k \leq K} \int_{k h}^{(k+1) h}\|\psi\|_{\infty}\|\nabla \eta\|_{\infty} \\
\quad \times\left(\int_{0}^{\infty}\left|U^{h}\left(x, y+g_{h}(x)\right)-U^{h}\left(k h+, y+g_{h}(x)\right)\right| d y\right) d x \\
\quad \leq O(1) \sum_{0 \leq k \leq K} \int_{k h}^{(k+1) h}(x-k h) d x \leq O(1) \operatorname{diam}(\operatorname{supp} \psi) h .
\end{aligned}
$$

Therefore, $I_{1} \rightarrow 0$ as $h \rightarrow 0$. 
From the construction of the approximate solutions, we have

$$
\begin{aligned}
\left|I_{2}\right| & \leq O(1) \sum_{k \geq 0} \int_{k h_{i}}^{(k+1) h}\left(\int_{0}^{\infty}\left|U^{h}\left(k h+, y+g_{h}(x)\right)-U^{h}\left(k h-, y+g_{h}(x)\right)\right||\psi| d y\right) d x \\
& \leq O(1)\|\bar{Z}\|_{\infty}\|\psi\|_{\infty} h \sum_{0 \leq k \leq K} \int_{k h}^{(k+1) h} e^{-L k h} d x \leq O(1)\|\bar{Z}\|_{\infty}\|\psi\|_{\infty} h \int_{0}^{\infty} e^{-L x} d x .
\end{aligned}
$$

Thus, $I_{2} \rightarrow 0$ as $h \rightarrow 0$.

Similarly, for $I_{3}$, we have

$$
\begin{aligned}
\left|I_{3}\right| & \leq O(1) \sum_{0 \leq k \leq K} \int_{k h}^{(k+1) h} \int_{0}^{\infty}\left|\psi\left(x, y+g_{h}(x)\right)-\psi\left(k h, y+g_{h}(x)\right)\right| d y d x \\
& \leq O(1) h \sum_{0 \leq k \leq K} \int_{k h}^{(k+1) h}\left\|\partial_{x} \psi\right\|_{\infty} d x \leq O(1) \operatorname{diam}(\operatorname{supp} \psi) h .
\end{aligned}
$$

Hence, it follows that $I_{3} \rightarrow 0$ as $h \rightarrow 0$. Therefore, $\Sigma_{1}^{2} \rightarrow 0$ as $h \rightarrow 0$.

For $\Sigma_{1}^{1}$, we have

$$
\begin{aligned}
\left|\Sigma_{1}^{1}\right| & \leq O(1)\|\psi\|_{\infty} \sum_{k \geq 0} \int_{k h}^{(k+1) h}\left(\int_{0}^{\infty}\left|U^{h}\left(x, y+g_{h}(x)\right)-U^{h}\left(k h+, y+g_{h}(x)\right)\right| d y\right) d x \\
& \leq O(1) \operatorname{diam}(\operatorname{supp} \psi) h .
\end{aligned}
$$

Thus, $\Sigma_{1}^{1} \rightarrow 0$ as $h \rightarrow 0$.

Finally, we also have

$$
\begin{aligned}
& \quad \iint_{\Omega}\left(\eta\left(W\left(U^{h}\right)\right) \psi_{x}+q\left(W\left(U^{h}\right)\right) \psi_{y}+\nabla_{W} \eta\left(W\left(U^{h}\right)\right) G\left(U^{h}\right) \psi\right) d x d y \\
& \quad-\iint_{\Omega_{h}}\left(\eta\left(W\left(U^{h}\right)\right) \psi_{x}+q\left(W\left(U^{h}\right)\right) \psi_{y}+\nabla_{W} \eta\left(W\left(U^{h}\right)\right) G\left(U^{h}\right) \psi\right) d x d y \mid \\
& \leq \iint_{\left\{\left(\Omega \backslash \Omega_{h}\right) \cup\left(\Omega_{h} \backslash \Omega\right)\right\} \cap\{x \geq 0\}}\left(\left|\eta\left(W\left(U^{h}\right)\right)\right|\left|\psi_{x}\right|+\left|q\left(W\left(U^{h}\right)\right)\right|\left|\psi_{y}\right|\right. \\
& \left.\quad+\left|\nabla_{W} \eta\left(W\left(U^{h}\right)\right) G\left(U^{h}\right)\right||\psi|\right) d x d y \\
& \rightarrow 0 \quad \text { as } h \rightarrow 0 .
\end{aligned}
$$

Moreover, by the Helly's compactness theorem, we can find a subsequence $h_{i} \rightarrow 0$ as $i \rightarrow \infty$ such that $U^{h_{i}} \rightarrow U$ in $L_{l o c}^{1}$ as $h_{i} \rightarrow 0$. Then, from (8.5)-(8.12), it follows that $U$ satisfies the entropy inequality (8.4), which implies that $U$ is an entropy solution of the initial-boundary value problem (1.1) and (1.10)-(1.11). This completes the proof.

Remark 8.1. In particular, we choose $\eta(W)= \pm W$ in (8.4) to conclude that the limit function $U=U(x, y)$ is a weak solution of the initial-boundary value problem (1.1) and (1.10) -(1.11) . 
By Corollary 7.1 and Proposition [8.1, we obtain the following existence theorem.

Theorem 8.1 (Existence). Under assumptions $\left(\mathbf{H}_{\mathbf{1}}\right)-\left(\mathbf{H}_{\mathbf{2}}\right)$ with $\arctan \left(g_{*}^{\prime}(x)\right) \in\left(\theta_{\text {crit }}, 0\right)$ for $x \in[0, \infty)$, there exist positive constants $\varepsilon, \delta_{0}$, and $C$ such that, if

$$
\left\|\bar{U}-U_{\infty}\right\|_{\infty}+\left|g_{+}^{\prime}(0)-g_{*+}^{\prime}(0)\right|+T V \cdot\{\bar{U}(\cdot)\}+T V \cdot\left\{\left(g_{+}^{\prime}-g_{*+}^{\prime}\right)(\cdot)\right\}<\varepsilon,
$$

then the initial-boundary value problem (1.1) and (1.10)-(1.11) admits a global entropy solution $U(x, y)$ that satisfies (1.12) in the sense of distributions. The solution is composed of a strong rarefaction wave that is a small perturbation of the complete reaction one. In addition,

$$
U(x, y) \in B V_{l o c}(\Omega) \cap D_{\delta_{0}}\left(U_{\infty}\right)
$$

and

$$
T V \cdot\{U(x, \cdot) ;[g(x), \infty)\} \leq C \quad \text { for any } x>0,
$$

where $D_{\delta_{0}}\left(U_{\infty}\right)$ is given by (2.17), and $\theta_{\text {crit }}$ is a critical angle defined by (2.18) .

\section{Asymptotic Behavior of the Solution}

In this section, our main purpose is to investigate the asymptotic behavior of entropy solutions $U(x, y)$. To achieve this, we need further estimates on $U^{\nu, h}(x, y)$.

Lemma 9.1. There exists a constant $M_{1}>0$, independent of $(\nu, h)$ and $U^{\nu, h}$, such that

$$
\sum_{\tau>0} E_{\nu, h}(\tau)<M_{1}
$$

where the summation is taken over all the interaction times, and $E_{\nu, h}(\tau)$ is defined as in (6.3).

Proof. First, by Proposition 6.1, we know that, for any $\tau \in[k h,(k+1) h), k \geq 0$,

$$
F\left(U^{\nu, h} ;(k+1) h-\right)-F\left(U^{\nu, h} ; k h+\right) \leq-\frac{1}{4} \sum_{k h}^{(k+1) h} E_{\nu, h}(\tau) .
$$

On the other hand, Lemma 7.7 implies that

$$
F\left(U^{\nu, h} ;(k+1) h+\right)-F\left(U^{\nu, h} ;(k+1) h-\right) \leq C_{4}\|\bar{Z}\|_{\infty} h\left(F\left(U^{\nu, h} ;(k+1) h-\right)+4\right)^{2} e^{-L k h} .
$$

Then combining these two inequalities and taking the summation $k$ yield

$$
\begin{aligned}
\sum_{k=0}^{\infty} \sum_{k h}^{(k+1) h} E_{\nu, h}(\tau) \leq & \sum_{k=0}^{\infty}\left(F\left(U^{\nu, h} ; k h+\right)-F\left(U^{\nu, h} ;(k+1) h+\right)\right) \\
& +C_{4}\|\bar{Z}\|_{\infty} h \sum_{k=0}^{\infty}\left(F\left(U^{\nu, h} ;(k+1) h-\right)+4\right)^{2} e^{-L k h} \\
\leq & O(1)\left(F\left(U^{\nu, h} ; k h+\right)+C_{4}\right)<\infty .
\end{aligned}
$$

Let $L_{\nu, h}\left(\Gamma_{h} ;[0, \infty)\right)$ be the summation of the strengths of weak waves leaving the approximate boundary $\Gamma_{h}$. Then, by Proposition 6.1 and Lemma 7.7, we have 
Lemma 9.2. There exists $M_{2}>0$, independent of $(\nu, h)$ and $U^{\nu, h}$, such that

$$
L_{\nu, h}\left(\Gamma_{h} ;[0, \infty)\right) \leq M_{2}\left(\sum_{\tau>0} E_{\nu, h}(\tau)+\|\bar{Z}\|_{\infty} h \sum_{k=0}^{\infty} e^{-L k h}\right) .
$$

Define

$$
B_{\nu, h}(x)=U^{\nu, h}\left(x, g_{h}(x)\right) .
$$

Then, by Lemmas 9.1 9.2, we have

Lemma 9.3. There exists a positive constant $M_{3}$ depending only on the system such that

$$
T V .\left\{B_{\nu, h}(\cdot) ;[0, \infty)\right\}<M_{3},
$$

which yields a subsequence $\left(\nu_{i}, h_{i}\right)$ such that

$$
B_{\nu_{i}, h_{i}}(x) \rightarrow B(x) \quad \text { as } \nu_{i} \rightarrow \infty \text { and } h_{i} \rightarrow 0
$$

for any $x \in[0, \infty)$, where $B(x) \in B V([0, \infty))$ and

$$
B(x) \cdot\left(g^{\prime}(x),-1,0,0,0\right)=0 .
$$

Let

$$
\begin{aligned}
& B_{\infty}=\lim _{x \rightarrow \infty} B(x+), \quad g_{\infty}^{\prime}=\lim _{x \rightarrow \infty} g^{\prime}(x+), \\
& B_{* \infty}=\lim _{x \rightarrow \infty} U^{*}\left(x, g_{*}(x)\right), \quad g_{* \infty}^{\prime}=\lim _{x \rightarrow \infty} g_{*}^{\prime}(x+)
\end{aligned}
$$

with

$$
B_{* \infty} \cdot\left(g_{* \infty}^{\prime},-1,0,0,0\right)=0,
$$

where $U^{*}(x, y)$ is the background solution stated in Corollary 3.1, and $y=g_{*}(x)$ is the non-perturbed Lipschitz convex wall. Then, owing to the properties of the background solution, we have

Lemma 9.4. There exists a constant $M_{4}>0$ depending only on the system such that

$$
g_{* \infty}^{\prime}-\lambda_{1}\left(B_{* \infty}\right)>M_{4} .
$$

Proof. By Corollary 3.1 and (9.7), we know that

$$
g_{* \infty}^{\prime}=\tan \theta_{\infty}^{*}, \quad \lambda_{1}\left(B_{* \infty}\right)=\tan \left(\theta_{\infty}^{*}-\theta_{\mathrm{ma}, \infty}^{*}\right) .
$$

Then

$$
g_{* \infty}^{\prime}-\lambda_{1}\left(B_{* \infty}\right)=\frac{\left(1+\tan ^{2} \theta_{\infty}^{*}\right) \tan \theta_{\mathrm{ma}, \infty}^{*}}{1+\tan \theta_{\infty}^{*} \tan \theta_{\mathrm{ma}, \infty}^{*}} .
$$

Since $0<\theta_{\mathrm{ma}, \infty}^{*}<\frac{\pi}{2}$ and $B_{* \infty} \in\left\{U: u>c_{*}, q<q_{*}\right\}$, then

$$
1+\tan \theta_{\infty}^{*} \tan \theta_{\mathrm{ma}, \infty}^{*}=\frac{\cos \left(\theta_{\infty}^{*}-\theta_{\mathrm{ma}, \infty}^{*}\right)}{\cos \theta_{\infty}^{*} \cos \theta_{\mathrm{ma}, \infty}^{*}}>0 .
$$

Therefore, we have

$$
g_{* \infty}^{\prime}-\lambda_{1}\left(B_{* \infty}\right)=\frac{\left(1+\tan ^{2} \theta_{\infty}^{*}\right) \tan \theta_{\mathrm{ma}, \infty}^{*}}{1+\tan \theta_{\infty}^{*} \tan \theta_{\mathrm{ma}, \infty}^{*}}>\tan \theta_{\mathrm{ma}, \infty}^{*},
$$

where we have used $\theta_{\text {crit }}<\theta_{\infty}^{*}<0$. This completes the proof. 
Lemma 9.5. For $\varepsilon$ given in Theorem 8.1 sufficiently small,

$$
g_{\infty}^{\prime}-\lambda_{1}\left(B_{\infty}\right)>\frac{M_{4}}{2}
$$

where $M_{4}$ is the same as in (9.8).

Proof. By the assumption of Theorem 8.1 , we have

$$
g_{\infty}^{\prime}-g_{* \infty}^{\prime}=O(1) \varepsilon .
$$

On the other hand, by (9.6)-(9.7), we have

$$
\theta\left(B_{\infty}\right)-\theta\left(B_{* \infty}\right)=O(1)\left(\arctan \left(g_{\infty}^{\prime}\right)-\arctan \left(g_{* \infty}^{\prime}\right)\right)=O(1)\left(g_{\infty}^{\prime}-g_{* \infty}^{\prime}\right)=O(1) \varepsilon
$$

Since

$$
\left|\left(J\left(q, \frac{q^{2}}{2}+\frac{c^{2}}{\gamma-1}\right)+\theta\right)\left(B_{\infty}\right)-\left(J\left(q, \frac{q^{2}}{2}+\frac{c^{2}}{\gamma-1}\right)+\theta\right)\left(B_{* \infty}\right)\right|=O(1) \varepsilon,
$$

and

$$
\begin{aligned}
& \left|\left(\frac{q^{2}}{2}+\frac{c^{2}}{\gamma-1}\right)\left(B_{\infty}\right)-\left(\frac{q^{2}}{2}+\frac{c^{2}}{\gamma-1}\right)\left(B_{* \infty}\right)\right|=O(1) \varepsilon, \\
& \left|\frac{p}{\rho^{\gamma}}\left(B_{\infty}\right)-\frac{p}{\rho^{\gamma}}\left(B_{* \infty}\right)\right|=O(1) \varepsilon
\end{aligned}
$$

then

$$
B_{\infty}-B_{* \infty}=O(1) \varepsilon
$$

which leads to

$$
\lambda_{1}\left(B_{\infty}\right)-\lambda_{1}\left(B_{* \infty}\right)=O(1) \varepsilon .
$$

Thus, for $\varepsilon$ sufficiently small and $M_{4}$ sufficiently large, we have

$$
g_{\infty}^{\prime}-\lambda_{1}\left(B_{\infty}\right)=g_{* \infty}^{\prime}-\lambda_{1}\left(B_{* \infty}\right)+O(1) \varepsilon>M_{4}+O(1) \varepsilon>\frac{M_{4}}{2} .
$$

This completes the proof.

As a direct consequence of Lemma 9.1, we have

Corollary 9.1. For any $0<\delta \ll \frac{g_{\infty}^{\prime}-\lambda_{1}\left(B_{\infty}\right)}{8}$ sufficiently small, there exists $x_{\delta}>0$ independent of $(\nu, h)$ such that

$$
\sum_{\tau>x_{\delta}} E_{\nu, h}(\tau)<\delta, \quad L_{\nu, h}\left(\Gamma_{h} ;\left[x_{\delta}, \infty\right)\right)<\delta,
$$

where $\delta>0$ depending only on the system.

Let $y=\psi_{g, k_{i}}^{5}(x)$ be the approximate 5-strong rarefaction front generated by the corner point $A_{k_{i}}=\left(x_{k_{i}}, g_{h}\left(x_{k_{i}}\right)\right), k_{i} \geq\left[\frac{x_{\delta}}{h}\right]+1$, which is denoted by $R_{5, k_{i}}^{b}$. Let $\Omega_{k_{i}}$ be the region between $R_{5, k_{i}}^{b}$ and $R_{5, k_{i}+1}^{b}$; see Fig. 9.1. 


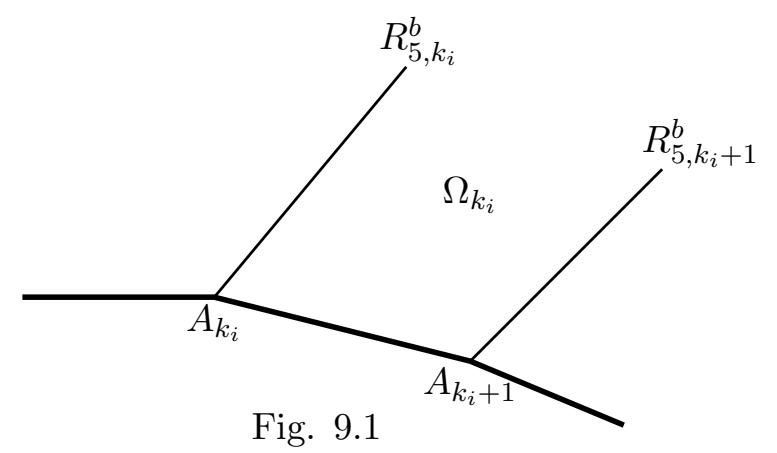

Let $L_{\nu, h}\left(\Omega_{k_{i}}\right)$ be the total amount of the strengths of weak waves entering $\Omega_{k_{i}}$. Then we have

Lemma 9.6. For $\varepsilon$ and $\delta$ given as in Theorem 8.1 and Corollary 9.1 ,

$$
L_{\nu, h}\left(\Omega_{k_{i}}\right)=O(1)(\varepsilon+\delta) .
$$

Proof. Using Proposition 6.1 and Lemma 7.7, we have

$$
\begin{aligned}
L_{\nu, h}\left(\Omega_{k_{i}}\right) \leq O(1)( & \sum_{\tau>x_{\delta}} E_{\nu, h}(\tau)+\|\bar{Z}\|_{\infty} h \sum_{k \geq\left[\frac{x_{\delta}}{h}\right]+1}^{\infty} e^{-L k h} \\
& \left.+L_{\nu, h}\left(\Gamma_{h} ;\left[x_{\delta}, \infty\right)\right)+F\left(U^{v, h} ; x_{\delta}+\right)\right),
\end{aligned}
$$

which leads to the desired result by Theorem 8.1, Lemma 9.2, and Corollary 9.1,

For any positive constant $M>0$, let

$$
M_{5}=\inf \left\{M: \lambda_{5}\left(B_{\nu, h}(x)\right)-\lambda_{1}\left(B_{\nu, h}(x)\right)>M, B_{\nu, h}(x) \in D_{\delta_{0}}\left(U_{\infty}\right)\right\},
$$

where $M_{5}>0$ is independent of $(\nu, h)$, and $B_{\nu, h}(x)$ is defined as in (9.3). Then

Lemma 9.7. For any points $\left(x_{j}, y_{j}\right) \in \Omega_{k_{i}}, j=1,2$,

$$
\lambda_{5}\left(U^{\nu, h}\left(x_{1}, y_{1}\right)\right)-\lambda_{1}\left(U^{\nu, h}\left(x_{2}, y_{2}\right)\right)>\frac{M_{5}}{2},
$$

where $M_{5}$ is defined as in (9.12).

Proof. First, by Lemma 9.6, we have

$$
\lambda_{5}\left(U^{\nu, h}\left(x_{1}, y_{1}\right)\right)-\lambda_{5}\left(B_{\nu, h}\left(x_{k_{i}}\right)\right)=O(1) L_{\nu, h}\left(\Omega_{k_{i}}\right)=O(1)(\varepsilon+\delta) .
$$

Similarly,

$$
\lambda_{1}\left(U^{\nu, h}\left(x_{2}, y_{2}\right)\right)-\lambda_{1}\left(B_{\nu, h}\left(x_{k_{i}}\right)\right)=O(1)(\varepsilon+\delta) .
$$

On the other hand, by (9.12),

$$
\lambda_{5}\left(B_{\nu, h}(x)\right)-\lambda_{1}\left(B_{\nu, h}(x)\right)>M_{5} .
$$

Then combining above estimates together yields

$$
\begin{aligned}
\lambda_{5}\left(U^{\nu, h}\left(x_{1}, y_{1}\right)\right)-\lambda_{1}\left(U^{\nu, h}\left(x_{2}, y_{2}\right)\right) & =\lambda_{5}\left(B_{\nu, h}(x)\right)-\lambda_{1}\left(B_{\nu, h}(x)\right)+O(1)(\varepsilon+\delta) \\
& =M_{5}+O(1)(\varepsilon+\delta),
\end{aligned}
$$

which leads to the desired result by choosing $\varepsilon$ and $\delta$ sufficiently small. 
Since $T V \cdot\left\{g^{\prime}(\cdot)\right\}<\infty$, then there exists $x_{*}>x_{\delta}$ such that

$$
g^{\prime}(x+)=g^{\prime}(\infty)+O(1) \delta \quad \text { for } x>x_{*} .
$$

Let $y=\chi_{\mathrm{g}}^{5}(x)$ be the maximal 5-generalized characteristic generated by point $\left(x_{*}, g\left(x^{*}\right)\right)$ on the bending wall $y=g(x)$. Then we have

Lemma 9.8. For any $x>x_{*}$,

$$
\sup \left\{|U(x, y)-B(x)| ; \quad\left(g(x), \chi_{\mathrm{g}}^{5}(x)\right)\right\}=O(1)(\varepsilon+\delta) .
$$

Proof. According to the construction of the approximate solutions, there exists a subsequence of approximate maximal 5 -generalized characteristics $\chi_{\mathrm{g}, \nu_{i}, h_{i}}^{5}(x)$ such that $\chi_{\mathrm{g}, \nu_{i}, h_{i}}^{5}(x) \rightarrow$ $\chi_{\mathrm{g}}^{5}(x)$ uniformly on every bounded interval as $\nu_{i} \rightarrow \infty$ and $h_{i} \rightarrow 0$. Then

$$
\begin{aligned}
& \sup \left\{\left|U^{\nu_{i}, h_{i}}(x, y)-B_{\nu_{i}, h_{i}}(x)\right| ;\left(g_{h_{i}}(x), \chi_{g, \nu_{i}, h_{i}}^{5}(x)\right)\right\} \\
& \leq O(1)\left(\sum_{\tau>x_{*}} E_{\nu_{i}, h_{i}}(\tau)+\|\bar{Z}\|_{\infty} h_{i} \sum_{k \geq\left[\frac{x_{*}}{h_{i}}\right]+1}^{\infty} e^{-L k h_{i}}\right. \\
& \left.\quad+L_{\nu_{i}, h_{i}}\left(\Gamma_{h_{i}} ;\left[x_{*}, \infty\right)\right)+F\left(U^{\nu_{i}, h_{i}} ; x_{*}+\right)\right) \\
& =O(1)(\varepsilon+\delta) .
\end{aligned}
$$

Then, passing to the limits for $\left(\nu_{i}, h_{i}\right)$ in the above, we obtain the expected result.

Lemma 9.9. For $g(x) \leq y \leq \chi_{\mathrm{g}}^{5}(x)$, when $\delta$ is sufficiently small,

$$
\lambda_{1}(U(x, y))-g_{\infty}^{\prime}<-\frac{M_{4}}{8} \quad \text { for } x>x_{*},
$$

where $M_{4}$ is given by (9.8).

Proof. By Lemma 9.7 and the construction of the approximate solutions, we deduce

$$
\begin{aligned}
& \lambda_{1}\left(U^{\nu, h}(x, y)\right)-\lambda_{1}\left(B_{\nu, h}(x)\right)=O(1)(\varepsilon+\delta), \\
& \lambda_{1}\left(B^{\nu, h}(x)\right)-\lambda_{1}\left(B_{\infty}\right)=O(1) L_{\nu, h}\left(\Gamma_{h} ;\left[x_{*}, \infty\right)\right)=O(1)(\varepsilon+\delta) .
\end{aligned}
$$

Then, by Lemma 9.5, choosing $\delta$ sufficiently small, we have

$$
\begin{aligned}
& \lambda_{1}\left(U^{\nu, h}(x)\right)-\lambda_{1}\left(B_{\infty}\right) \\
& =\left(\lambda_{1}\left(U^{\nu, h}(x)\right)-\lambda_{1}\left(B^{\nu, h}(x)\right)\right)+\left(\lambda_{1}\left(B^{\nu, h}(x)\right)-\lambda_{1}\left(B_{\infty}\right)\right)+\left(\lambda_{1}\left(B_{\infty}\right)-g_{\infty}^{\prime}\right) \\
& =\lambda_{1}\left(B_{\infty}\right)-g_{\infty}^{\prime}+O(1)(\varepsilon+\delta)<-\frac{g_{\infty}^{\prime}-\lambda_{1}\left(B_{\infty}\right)}{2}+O(1) \varepsilon \\
& <-\frac{M_{4}}{4}+O(1) \varepsilon<-\frac{M_{4}}{8} .
\end{aligned}
$$

Finally, passing to the limits for $U^{\nu, h}$ in the above by choosing a subsequence denoted by itself, we conclude the proof.

Lemma 9.10. $U(x, y)$ and $\chi_{\mathrm{g}}^{5}(x)$ satisfy

$$
\lim _{x \rightarrow \infty} T V \cdot\left\{\left(\frac{v}{u}, p\right)(x, \cdot) ;\left(g(x), \chi_{\mathrm{g}}^{5}(x)\right)\right\}=0 .
$$


Proof. Let $U^{\nu_{i}, h_{i}}$ be the sequence of approximate solutions stated in Propositions 8.18 .2 , and let the corresponding term $E_{\nu_{i}, h_{i}}(\tau)$ be the quantity defined in (6.3). As in [20], we denote $d E_{\nu_{i}, h_{i}}(\tau)$ as the measures assigning $E_{\nu_{i}, h_{i}}(\tau)$ at the interaction line $x=\tau$. Then, by Lemma 9.1, we can select a subsequence (still denoted by) $E_{\nu_{i}, h_{i}}(\tau)$ such that

$$
d E_{\nu_{i}, h_{i}}(\tau) \rightarrow d E(\tau) \quad \text { as } \nu_{i} \rightarrow \infty \text { and } h_{i} \rightarrow 0
$$

with $E(\tau)<\infty$. Therefore, for $\hat{\delta}<\delta$ sufficiently small, we can choose $x_{\hat{\delta}}>x_{*}$ independent of $U^{\nu_{i}, h_{i}}$ and $\left(\nu_{i}, h_{i}\right)$ such that

$$
\sum_{\tau>x_{\hat{\delta}}} E_{\nu_{i}, h_{i}}(\tau)<\hat{\delta}
$$

Let $X_{\hat{\delta}}^{1}=\left(x_{\hat{\delta}}, \chi_{g, \nu_{i}, h_{i}}^{5}\left(x_{\hat{\delta}}\right)\right)$ and $X_{\hat{\delta}}^{5}=\left(x_{\hat{\delta}}, g_{h}\left(x_{\hat{\delta}}\right)\right)$ be the two points lying in the approximate 5-maximal characteristic $y=\chi_{g, \nu_{i}, h_{i}}^{5}(x)$ and the approximate boundary $y=g_{h}(x)$, respectively. Let $\chi_{\nu_{i}, h_{i}}^{j}$ be the approximate $j$-generalized characteristic generated from $X_{\hat{\delta}}^{j}$ for $j=1,5$, respectively. According to the construction of the approximate solutions, there exist constants $\hat{M}_{j}>0, j=1,5$, independent of $U^{\nu_{i}, h_{i}}$ and $\left(\nu_{i}, h_{i}\right)$ such that

$$
\left|\chi_{\nu_{i}, h_{i}}^{j}\left(x_{1}\right)-\chi_{\nu_{i}, h_{i}}^{j}\left(x_{2}\right)\right| \leq \hat{M}_{j}\left(\left|x_{1}-x_{2}\right|+h_{i}+\frac{1}{2^{\nu_{i}}}\right) \quad \text { for } x_{1}, x_{2}>x_{\hat{\delta}} .
$$

Then we can choose a subsequence (still denoted by) $\left\{\nu_{i}\right\}$ and $\left\{h_{i}\right\}$ such that

$$
\chi_{\nu_{i}, h_{i}}^{j}(x) \rightarrow \chi^{j}(x) \quad \text { as } i \rightarrow \infty
$$

for some $\chi^{j} \in$ Lip with $\left(\chi^{j}\right)^{\prime}$ bounded.

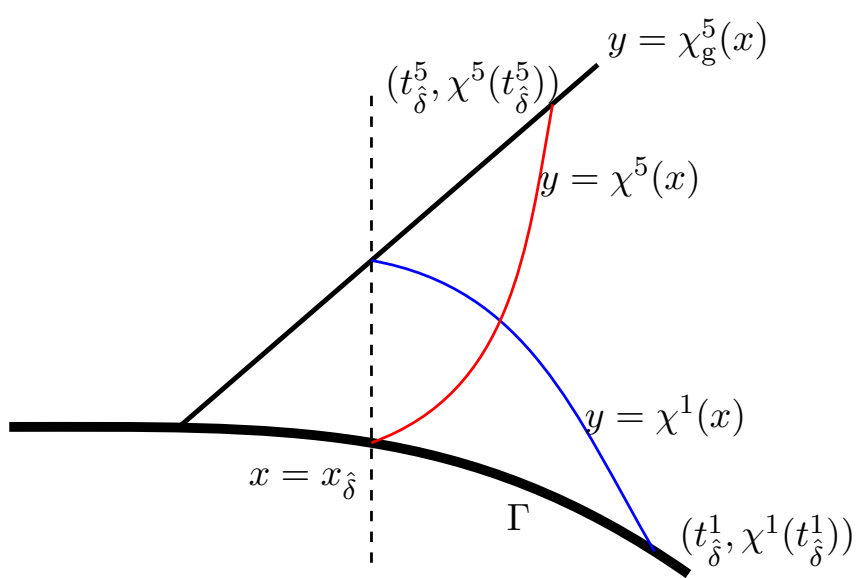

Fig. 9.2. The characteristics $\chi^{1}$ and $\chi^{5}$ intersect with $\Gamma$ and $y=\chi_{\mathrm{g}}^{5}(x)$, respectively

According to Lemma 9.9, $\chi^{1}$ can intersect with boundary $\Gamma$, whose intersection point is denoted by $\left(t_{\hat{\delta}}^{1}, \chi^{1}\left(t_{\hat{\delta}}^{1}\right)\right)$. Also, let $\chi^{5}$ intersect with $y=\chi_{\mathrm{g}}^{5}(x)$ at point $\left(t_{\hat{\delta}}^{5}, \chi^{5}\left(t_{\hat{\delta}}^{5}\right)\right)$; see Fig. 9.2. Since $\frac{v^{\nu_{i}}, h_{i}}{u^{\nu_{i}, h_{i}}}$ and $p^{\nu_{i}, h_{i}}$ are invariant across the $k$-contact discontinuities for $k=2,3,4$. Then we can do as in [20, 29] by applying the approximate conservation laws, we deduce 

that, for $x>2\left(t_{\hat{\delta}}^{1}+t_{\hat{\delta}}^{5}\right)$,

$$
T V \cdot\left\{\left(\frac{v^{\nu_{i}, h_{i}}}{u^{\nu_{i}, h_{i}}}, p^{\nu_{i}, h_{i}}\right)(x, \cdot) ;\left(g(x), \chi_{\mathrm{g}}^{5}(x)\right)\right\} \leq C_{8} \hat{\delta},
$$

where the positive constant $C_{8}$ is independent of $\left(\hat{\delta}, \nu_{i}, h_{i}\right)$ and $U^{\nu_{i}, h_{i}}$. Therefore, by Proposition 8.1 8.2 and taking the limit as $\nu_{i} \rightarrow \infty$ and $h_{i} \rightarrow 0$, it follows that

$$
T V \cdot\left\{\left(\frac{v}{u}, p\right)(x, \cdot) ;\left(g(x), \chi_{\mathrm{g}}^{5}(x)\right)\right\} \leq C_{8} \hat{\delta} \quad \text { for } x>2\left(t_{\hat{\delta}}^{1}+t_{\hat{\delta}}^{5}\right) .
$$

By similar arguments for $y>\chi_{\mathrm{g}}^{5}(x)$, we have

Lemma 9.11. The following hold:

$$
\begin{aligned}
& \lim _{x \rightarrow \infty} T V \cdot\left\{\left(J\left(q, \frac{q^{2}}{2}+\frac{c^{2}}{\gamma-1}\right)+\theta, \frac{q^{2}}{2}+\frac{c^{2}}{\gamma-1}\right)(U(x, \cdot)) ;\left(\chi_{\mathrm{g}}^{5}(x), \infty\right)\right\}=0, \\
& \lim _{x \rightarrow \infty} T V \cdot\left\{\left(\frac{p}{\rho^{\gamma}}, Z\right)(x, \cdot) ;\left(\chi_{\mathrm{g}}^{5}(x), \infty\right)\right\}=0 .
\end{aligned}
$$

Theorem 9.1 (Asymptotic behavior). Let $\chi_{\mathrm{g}}^{5}(x)$ be stated as above.

(i) Let $g_{\infty}^{\prime}=\lim _{x \rightarrow \infty} g^{\prime}(x+)$. Then

$$
\lim _{x \rightarrow \infty} \sup \left\{\left|\frac{v}{u}(x, \cdot)-g_{\infty}^{\prime}\right| ; \quad\left(g(x), \chi_{\mathrm{g}}^{5}(x)\right)\right\}=0,
$$

and there exists a constant $p_{+}$such that

$$
\lim _{x \rightarrow \infty} \sup \left\{\left|p(x, \cdot)-p_{+}\right| ;\left(g(x), \chi_{\mathrm{g}}^{5}(x)\right)\right\}=0 .
$$

(ii) There exists a constant state $U^{\infty}=\left(u^{\infty}, v^{\infty}, p^{\infty}, \rho^{\infty}, 0\right)$ such that

$$
\begin{aligned}
& \lim _{x \rightarrow \infty} \sup \left\{\left|\left(J\left(q, \frac{q^{2}}{2}+\frac{c^{2}}{\gamma-1}\right)+\theta\right)(U(x, \cdot))-\left(J\left(q, \frac{q^{2}}{2}+\frac{c^{2}}{\gamma-1}\right)+\theta\right)\left(U^{\infty}\right)\right| ;\left(\chi_{\mathrm{g}}^{5}(x), \infty\right)\right\} \\
& \quad=0 \\
& \lim _{x \rightarrow \infty} \sup \left\{\left|\left(\frac{q^{2}}{2}+\frac{c^{2}}{\gamma-1}\right)(U(x, \cdot))-\left(\frac{q^{2}}{2}+\frac{c^{2}}{\gamma-1}\right)\left(U^{\infty}\right)\right| ; \quad\left(\chi_{\mathrm{g}}^{5}(x), \infty\right)\right\}=0, \\
& \lim _{x \rightarrow \infty} \sup \left\{\left|\left(\frac{p}{\rho^{\gamma}}\right)(x, \cdot)-\frac{p^{\infty}}{\left(\rho^{\infty}\right)^{\gamma}}\right|+|Z(x, \cdot)| ;\left(\chi_{\mathrm{g}}^{5}(x), \infty\right)\right\}=0 .
\end{aligned}
$$

Proof. Let $U^{\nu_{i}, h_{i}}$ be the approximate solutions of problem (1.1) and (1.10)-(1.11), where $\left(\nu_{i}, h_{i}\right)$ is the sequence given as in Propositions 8.1 8.2. From the construction of the approximate solutions, we have

$$
\left.\frac{v^{\nu_{i}, h_{i}}}{u^{\nu_{i}, h_{i}}}\right|_{\Gamma_{h_{i}}}=g_{h_{i}}^{\prime}(x) \quad \text { for some } x \in\left(k h_{i},(k+1) h_{i}\right) .
$$

Then we can choose some $x_{\hat{\delta}}>x_{*}$ such that, for $x>2 x_{\hat{\delta}}$,

$$
\left|g_{h_{i}}^{\prime}(x)-g_{\infty}^{\prime}\right|<\frac{\hat{\delta}}{2}
$$


Thus, by Lemma 9.10, we have

$$
\begin{aligned}
& \sup \left\{\left|\frac{v^{\nu_{i}, h_{i}}}{u^{\nu_{i}, h_{i}}}(x, y)-g_{\infty}^{\prime}\right| ;\left(g_{h_{i}}(x), \chi_{g, \nu_{i}, h_{i}}^{5}(x)\right)\right\} \\
& \leq T V \cdot\left\{\frac{v^{\nu_{i}, h_{i}}}{u^{\nu_{i}, h_{i}}}(x, \cdot) ;\left(g_{h_{i}}(x), \chi_{g, \nu_{i}, h_{i}}^{5}(x)\right)\right\}+\left|g_{h_{i}}^{\prime}(x)-g_{\infty}^{\prime}\right| \leq \hat{\delta} \quad \text { for } x>2 x_{\hat{\delta}} .
\end{aligned}
$$

Then, letting $i \rightarrow \infty$ and by Propositions 8.1 8.2, we obtain (9.19).

To prove (9.20), let $p_{+}=\lim _{x \rightarrow \infty} p(x, g(x))$. Then

$$
\begin{aligned}
& \sup \left\{\left|p^{\nu_{i}, h_{i}}(x, \cdot)-p_{+}\right| ;\left(g_{h_{i}}(x), \chi_{g, \nu_{i}, h_{i}}^{5}(x)\right)\right\} \\
& \leq T . V \cdot\left\{p^{\nu_{i}, h_{i}}(x, \cdot) ;\left(g_{h_{i}}(x), \chi_{g, \nu_{i}, h_{i}}^{5}(x)\right)\right\}+\left|p^{\nu_{i}, h_{i}}\left(x, g_{h_{i}}(x)\right)-p_{+}\right| \leq \hat{\delta} .
\end{aligned}
$$

Thus, for $x>2 x_{\hat{\delta}}$, letting $i \rightarrow \infty$, and by Propositions 8.1$] 8.2$, we can obtain the desired result.

Now we consider (ii). Let $U^{\infty}=\lim _{x \rightarrow \infty} U(x, \infty)$. Then

$$
\begin{aligned}
& \sup \left|\left(J\left(q, \frac{q^{2}}{2}+\frac{c^{2}}{\gamma-1}\right)+\theta\right)\left(U^{\nu_{i}, h_{i}}(x, \cdot)\right)-\left(J\left(q, \frac{q^{2}}{2}+\frac{c^{2}}{\gamma-1}\right)+\theta\right)\left(U^{\infty}\right)\right| \\
& \leq T V \cdot\left\{\left(J\left(q, \frac{q^{2}}{2}+\frac{c^{2}}{\gamma-1}\right)+\theta\right)\left(U^{\nu_{i}, h_{i}}(x, \cdot)\right) ;\left(\chi_{g, \nu_{i}, h_{i}}^{5}(x), \infty\right)\right\} \\
& \quad+\left|\left(J\left(q, \frac{q^{2}}{2}+\frac{c^{2}}{\gamma-1}\right)+\theta\right)\left(U^{\nu_{i}, h_{i}}(x, \infty)\right)-\left(J\left(q, \frac{q^{2}}{2}+\frac{c^{2}}{\gamma-1}\right)+\theta\right)\left(U^{\infty}\right)\right| \leq \hat{\delta}
\end{aligned}
$$

for $x>2 x_{\hat{\delta}}$. Then, letting $i \rightarrow \infty$, and by Propositions 8.1$] 8.2$, we obtain the desire result. The proof of the others in (9.20) is similar. This completes the proof.

\section{Appendix}

In this section, we complete the proof of Lemma 2.1 by several lemmas.

Lemma 10.1. For $u>c$,

$$
\cos \left(\theta+(-1)^{\frac{j+3}{4}} \theta_{\mathrm{ma}}\right)>0 \quad \text { for } j=1,5 .
$$

Proof. Since $\left(u \sqrt{q^{2}-c^{2}}\right)^{2}-(v c)^{2}=q^{2}\left(u^{2}-c^{2}\right)>0$. Then, by direct computation, we have

$$
q^{2} \cos \left(\theta+(-1)^{\frac{j+3}{4}} \theta_{\text {ma }}\right)=u \sqrt{q^{2}-c^{2}}-(-1)^{\frac{j+3}{4}} v c>0, \quad j=1,5 .
$$

Lemma 10.2. For $u>c$,

$$
\begin{aligned}
& \nabla_{(u, v)} q=(\cos \theta, \sin \theta), \quad \nabla_{(u, v)} \theta=\frac{1}{q}(-\sin \theta, \cos \theta), \\
& \nabla_{(u, v)} \theta_{\mathrm{ma}}=-\frac{\tan \left(\theta_{\mathrm{ma}}\right)}{q}(\cos \theta, \sin \theta), \quad \nabla_{(p, \rho)} \theta_{\mathrm{ma}}=\frac{\tan \left(\theta_{\mathrm{ma}}\right)}{2 \rho c^{2}}\left(\gamma,-c^{2}\right) .
\end{aligned}
$$

Proof. Since $\theta_{\mathrm{ma}}=\arctan \left(\frac{c}{\sqrt{q^{2}-c^{2}}}\right)$, then

$$
\frac{\partial \theta_{\mathrm{ma}}}{\partial u}=\frac{q^{2}-c^{2}}{q^{2}} \frac{\partial}{\partial u}\left(\frac{c}{\sqrt{q^{2}-c^{2}}}\right)=-\frac{c}{q \sqrt{q^{2}-c^{2}}} \cos \theta=-\frac{\cos \theta \tan \left(\theta_{\mathrm{ma}}\right)}{q} .
$$


Similarly for $\frac{\partial \theta_{\text {ma }}}{\partial v}$. Notice that $c^{2}=\frac{\gamma p}{\rho}$, then $\frac{\partial c}{\partial p}=\frac{\gamma}{2 \rho c}$ and $\frac{\partial c}{\partial \rho}=-\frac{c}{2 \rho}$. Then

$$
\begin{aligned}
& \frac{\partial \theta_{\mathrm{ma}}}{\partial p}=\frac{q^{2}-c^{2}}{q^{2}} \frac{\partial}{\partial p}\left(\frac{c}{\sqrt{q^{2}-c^{2}}}\right)=\frac{q^{2}-c^{2}}{q^{2}} \frac{q^{2}}{\left(q^{2}-c^{2}\right)^{\frac{3}{2}}} \frac{\partial c}{\partial p}=\frac{\gamma}{2 \rho c^{2}} \tan \left(\theta_{\mathrm{ma}}\right), \\
& \frac{\partial \theta_{\mathrm{ma}}}{\partial \rho}=\frac{q^{2}-c^{2}}{q^{2}} \frac{\partial}{\partial \rho}\left(\frac{c}{\sqrt{q^{2}-c^{2}}}\right)=\frac{q^{2}-c^{2}}{q^{2}} \frac{q^{2}}{\left(q^{2}-c^{2}\right)^{\frac{3}{2}}} \frac{\partial c}{\partial \rho}=-\frac{1}{2 \rho} \tan \left(\theta_{\mathrm{ma}}\right) .
\end{aligned}
$$

The others can be proved in the same way. This completes the proof.

Lemma 10.3. For $u>c$,

$$
\begin{aligned}
& \nabla_{(u, v)} \lambda_{j}=\frac{\sec ^{2}\left(\theta+(-1)^{\frac{j+3}{4}} \theta_{\mathrm{ma}}\right)}{\sqrt{q^{2}-c^{2}}}\left(-\sin \left(\theta+(-1)^{\frac{j+3}{4}} \theta_{\mathrm{ma}}\right), \cos \left(\theta+(-1)^{\frac{j+3}{4}} \theta_{\mathrm{ma}}\right)\right) \\
& \nabla_{(p, \rho)} \lambda_{j}=\frac{(-1)^{\frac{j+3}{4}}}{2 \rho c^{2}} \sec ^{2}\left(\theta+(-1)^{\frac{j+3}{4}} \theta_{\mathrm{ma}}\right) \tan \left(\theta_{\mathrm{ma}}\right)\left(\gamma,-c^{2}\right) \\
& \frac{\partial \lambda_{j}}{\partial Z}=0
\end{aligned}
$$

for $j=1,5$, and

$$
\nabla_{(u, v)} \lambda_{i}=\frac{\sec ^{2} \theta \sin \theta}{q}(-1,1), \quad \frac{\partial \lambda_{i}}{\partial p}=\frac{\partial \lambda_{i}}{\partial \rho}=\frac{\partial \lambda_{i}}{\partial Z}=0 \quad \text { for } i=2,3,4 .
$$

Proof. We only consider the case $j=1$, since $j=2$ and $i=2,3,4$ can be carried out in the same way. By Lemma 10.2, we have

$$
\begin{aligned}
\frac{\partial \lambda_{1}}{\partial u} & =\frac{\partial \lambda_{1}}{\partial \theta} \frac{\partial \theta}{\partial u}+\frac{\partial \lambda_{1}}{\partial \theta_{\mathrm{ma}}} \frac{\partial \theta_{\mathrm{ma}}}{\partial u}=\sec ^{2}\left(\theta-\theta_{\mathrm{ma}}\right)\left(-\frac{\sin \theta}{q}\right)+\frac{\cos \theta \tan \left(\theta_{\mathrm{ma}}\right)}{q} \sec ^{2}\left(\theta-\theta_{\mathrm{ma}}\right) \\
& =-\frac{1}{\sqrt{q^{2}-c^{2}}} \sec ^{2}\left(\theta-\theta_{\mathrm{ma}}\right) \sin \left(\theta-\theta_{\mathrm{ma}}\right), \\
\frac{\partial \lambda_{1}}{\partial v} & =\frac{\partial \lambda_{1}}{\partial \theta} \frac{\partial \theta}{\partial v}+\frac{\partial \lambda_{1}}{\partial \theta_{\mathrm{ma}}} \frac{\partial \theta_{\mathrm{ma}}}{\partial v}=\sec ^{2}\left(\theta-\theta_{\mathrm{ma}}\right) \frac{\cos \theta}{q}+\frac{\sin \theta \tan \left(\theta_{\mathrm{ma}}\right)}{q} \sec ^{2}\left(\theta-\theta_{\mathrm{ma}}\right) \\
& =\frac{1}{\sqrt{q^{2}-c^{2}}} \sec ^{2}\left(\theta-\theta_{\mathrm{ma}}\right) \cos \left(\theta-\theta_{\mathrm{ma}}\right), \\
\frac{\partial \lambda_{1}}{\partial p} & =\frac{\partial \lambda_{1}}{\partial \theta_{\mathrm{ma}}} \frac{\partial \theta_{\mathrm{ma}}}{\partial p}=-\frac{\gamma}{2 \rho c^{2}} \sec ^{2}\left(\theta-\theta_{\mathrm{ma}}\right) \tan \left(\theta_{\mathrm{ma}}\right), \\
\frac{\partial \lambda_{1}}{\partial \rho} & =\frac{\partial \lambda_{1}}{\partial \theta_{\mathrm{ma}}} \frac{\partial \theta_{\mathrm{ma}}}{\partial \rho}=\frac{1}{2 \rho} \sec ^{2}\left(\theta-\theta_{\mathrm{ma}}\right) \tan \left(\theta_{\mathrm{ma}}\right),
\end{aligned}
$$

and clearly $\frac{\partial \lambda_{j}}{\partial Z}=0$. This completes the proof. 
Proof of Lemma 2.1. We consider Case $j=1$, since Case $j=5$ can be done in the same way. By Lemma 10.1 and direct computation, we have

$$
\begin{aligned}
\nabla_{U} \lambda_{1} \cdot \tilde{r}_{1}= & -\frac{\partial \lambda_{1}}{\partial u} \tan \left(\theta-\theta_{\mathrm{ma}}\right)+\frac{\partial \lambda_{1}}{\partial v}-\frac{\partial \lambda_{1}}{\partial p} \rho q \sec \left(\theta-\theta_{\mathrm{ma}}\right) \sin \left(\theta_{\mathrm{ma}}\right) \\
& -\frac{\partial \lambda_{1}}{\partial \rho} \frac{\rho q}{c^{2}} \sec \left(\theta-\theta_{\mathrm{ma}}\right) \sin \left(\theta_{\mathrm{ma}}\right) \\
= & \frac{1}{\sqrt{q^{2}-c^{2}}} \sec ^{2}\left(\theta-\theta_{\mathrm{ma}}\right) \sin \left(\theta-\theta_{\mathrm{ma}}\right) \tan \left(\theta-\theta_{\mathrm{ma}}\right) \\
& +\frac{1}{\sqrt{q^{2}-c^{2}}} \sec ^{2}\left(\theta-\theta_{\mathrm{ma}}\right) \cos \left(\theta-\theta_{\mathrm{ma}}\right) \\
& +\frac{\gamma}{2 \rho c^{2}} \sec ^{2}\left(\theta-\theta_{\mathrm{ma}}\right) \tan \left(\theta_{\mathrm{ma}}\right) \rho q \sec \left(\theta-\theta_{\mathrm{ma}}\right) \sin \left(\theta_{\mathrm{ma}}\right) \\
& -\frac{1}{2 \rho} \sec ^{2}\left(\theta-\theta_{\mathrm{ma}}\right) \tan \left(\theta_{\mathrm{ma}}\right) \frac{\rho q}{c^{2}} \sec \left(\theta-\theta_{\mathrm{ma}}\right) \sin \left(\theta_{\mathrm{ma}}\right) \\
= & \frac{\gamma+1}{\sqrt{q^{2}-c^{2}}} \sec ^{3}\left(\theta-\theta_{\mathrm{ma}}\right)>0 .
\end{aligned}
$$

Similarly, for $j=5$,

$$
\nabla_{U} \lambda_{5} \cdot \tilde{r}_{5}=\frac{\gamma-1}{\sqrt{q^{2}-c^{2}}} \sec ^{3}\left(\theta+\theta_{\mathrm{ma}}\right)>0 .
$$

In a similar way, we can prove

$$
\nabla_{U} \lambda_{j} \cdot \tilde{r}_{j}=0, \quad j=2,3,4 .
$$

This completes the proof.

Acknowledgements. The research of Gui-Qiang Chen was supported in part by the UK EPSRC Award to the EPSRC Centre for Doctoral Training in PDEs (EP/L015811/1) and the Royal Society-Wolfson Research Merit Award (UK). Yongqian Zhang was supported in part by NSFC Project 11421061, NSFC Project 11031001, NSFC Project 11121101, the 111 Project B08018 (China), and by Natural Science Foundation of Shanghai 15ZR1403900.

Conflict of Interest. The authors declare that they have no conflict of interest.

\section{REFERENCES}

[1] D. Amadori, Initial boundary value problem for nonlinear systems of conservation laws. NoDEA. 4 (1997), 1-42.

[2] D. Amadori, L. Gosse, and G. Guerra, Global BV entropy solutions and uniqueness for hyperbolic system of balance laws. Arch. Rational Mech. Anal. 162 (2002), 327-366.

[3] A. Bourlioux, A. Majda, and V. Roytburd, Theoretical and numerical structure for unstable onedimensional detonations. SIAM J. Appl. Math. 51 (1991), 303-343.

[4] A. Bressan, Hyperbolic Systems of Conservation Laws: The One-Dimensional Cauchy Problem. Oxford University Press Inc.: New York, 2000.

[5] G.-Q. Chen and T. Li, Well-posedness for two-dimnsional steady supersonic Euler flows past a Lipschitz wedge. J. Diff. Eqs. 244 (2008), 1521-1550.

[6] G.-Q. Chen and D. Wagner, Global entropy solutions to exothermically reacting, compressible Euler equations. J. Diff. Eqs. 191 (2003), 277-322. 
[7] G.-Q. Chen, C. Xiao, and Y. Zhang, Existence of entropy solutions to two-dimensional steady exothermically reacting Euler equations. Acta Math. Sci. 34B(1) (2014), 1-38.

[8] G.-Q. Chen, Y. Zhang, and D. Zhu, Existence and stability of supersonic Euler flows past Lipschitz wedges. Arch. Rational Mech. Anal. 181 (2006), 261-310.

[9] S. Chen, Supersonic flow past a concave wedge. Sci. China. 10A(27) (1997), 903-910.

[10] S. Chen and D. Li, Supersonic flow past a symmetrically curved cone. Indiana Univ. Math. J. 49(4) (2000), 1411-1435.

[11] S. Chen, Z. Xin, and H. Yin, Global shock waves for the supersonic flow past a perturbed cone. Commun. Math. Phys. 228 (2002), 47-84.

[12] A. J. Chorin, Random choice methods with application to reacting gas flow. J. Comput. Phys. 25 (1977), 253-272.

[13] N. Costanzino, H. K. Jenssen, G. Lyng, and M. Williams, Existence and stability of curved multidimensional detonation fronts. Indiana Univ. Math. J. 56 (2007), 1405-1461.

[14] R. Courant and K. Friedrichs, Supersonic Flow and Shock Waves. Interscience Publishers Inc.: New York, 1948.

[15] D. Cui and H. Yin, Global conic shock wave for the steady supersonic flow past a cone: Polytropic cas. J. Diff. Eqs. 246 (2009), 641-669.

[16] J.J. Erpenbeck, Stability of idealized one-reaction detonations. Phys. Fluids, 7 (1964), 684-696.

[17] W. Fickett, Detonation in miniature. Amer. J. Phys. 47 (1979), 1050-1059.

[18] W. Fickett and W. W. Wood, Flow calculation for pulsating one-dimensional detonation. Phys. Fluids, 9 (1966), 903-916.

[19] J. Glimm, Solutions in the large for nonlinear hyperbolic systems of wquations. Comm. Pure Appl. Math. 18 (1965), 697-715.

[20] J. Glimm and P. D. Lax, Decay of Solutions of Systems of Hyperbolic Conservation Laws. Mem. Amer. Math. Soc. 101 (1970).

[21] P. D. Lax, Hyperbolic system of conservation laws II. Comm. Pure Appl. Math. 10 (1957), 537-566.

[22] H. I. Lee and D. Scott Stewart, Calculation of linear detonation instability: one dimensional instability of plane detonation. J. Fluid Mech. 216 (1990), 103-132.

[23] M. Lewicka, Well-posedness for hyperbolic systems of conservation laws with large BV data. Arch. Rational Mech. Anal. 173 (2004), 415-445.

[24] M. Lewicka, Stability conditions for strong rarefaction waves. SIAM J. Math. Anal. 36 (2005), 13461369.

[25] M. Lewicka, Lyapunov functional for solutions of systems of conservation laws containing a strong rarefaction. SIAM J. Math. Anal. 36 (2005), 1371-1399.

[26] M. Lewicka and K. Trivisa, On the $L^{1}$ well posedness of systems of conservation laws near solutions containing two large shocks. J. Diff. Eqs. 179 (2002), 133-177.

[27] J.-Q. Li and P. Zhang, The transition from Zeldovich-von Neumann-Döring to ChapmanCJouget theories for a nonconvex scalar combustion model. SIAM J. Math. Anal. 34 (2003), 675-699.

[28] W. Lien and T-P. Liu, Nonlinear stability of a self-similar 3-D gas flow. Commun. Math. Phys. 304 (1999), 524-549.

[29] T.-P. Liu, Large-time behavior of initial and initial-boundary value problems of a general systemof hyperbolic conservation laws. Commun. Math. Phys. 55 (1977), 163-177.

[30] T.-P. Liu and L.-A. Ying, Nonlinear stability of strong denotations for a viscous combustion model. SIAM J. Math. Anal. 26 (1995), 519-528.

[31] A. Majda, A qualitative model for dynamic combustion. SIAM J. Appl. Math. 41 (1981), 70-93.

[32] J. von Neumann, Theory of detonation waves. In: Collected Works of John Von Neumann, Vol. 6, A. J. Taub (Ed.), New York: Macmillan, 1956.

[33] A. K. Oppenheim and R.I. Soloukhin, Experiments in gasdynamics of explosions. Ann. Rev. Fluid Mech. 5 (1973), 31-58.

[34] R. R. Rosales and A. Majda, Weakly nonlinear detonation waves. SIAM J. Appl. Math. 43 (1983), 1086-1118.

[35] S. Schochet, Sufficient conditions for local existence via Glimm's scheme for large BV data. J. Diff. Eqs. 89 (1991), 317-354. 
[36] W. C. Sheng and T. Zhang, Structural stability of the Riemann problem for a scalar non-convex C-J combustion model. Discrete Contin. Dynam. Systems. 25(2) (2009), 651-667.

[37] J. Smoller, Shock Waves and Reaction-Diffusion Equations. 2nd Edition, New York: Springer, 1994.

[38] Z. H. Teng, A. J. Chorin, and T.-P. Liu, Riemann problems for reacting gas with applications to transition. SIAM J. Appl. Math. 42 (1982), 964-981.

[39] D. Wagner, The existence and behavior of viscous structure for plane detonation waves. SIAM J. Math. Anal. 20 (1989), 1035-1054.

[40] Z. Wang and H. Yin, Local structural stability of a multidimensional centered rarefaction wave for the three-dimensional steady supersonic Euler flow around a sharp corner. SIAM J. Math. Anal. 42(4) (2010), 1639-1687.

[41] Z. Wang and Y. Zhang, Steady supersonic flow past a curved cone. J. Diff. Eqs. 247 (2009), 1817-1850.

[42] F. A. Williams, Combusition Theory. 2nd Edition, 1985.

[43] L. A. Ying and Z. Teng, Riemann problem for a reaction and convection hyperbolic system. Approx. Theory Appl. 1 (1984), 95-122.

[44] T. Zhang and Y. X. Zheng, Riemann problem for gas dynamic combustion. J. Diff. Eqs. 77 (1989), 203-230.

[45] Y. Zhang, Steady supersonic flow over a bending wall. Nonlinear Analysis: Real World Applications. 12 (2011), 167-189.

[46] Y. Zhang, Global existence of steady supersonic potential flow past a curved wedge with piecewise smooth boundary. SIAM J. Math. Anal. 31(1) (1999), 166-183.

[47] Y. Zhang, Steady supersonic flow past an almost straight wedge with large vertex angle. J. Diff. Eqs. 192(1) (2003), 1-46.

[48] Y. Zhang, Asymptotic behaviour of steady supersonic flow past a wedge. Chinese Ann. Math. 26B(3) (2005), 379-392.

Mathematical Institute, University of Oxford, Oxford, OX2 6GG, UK

E-mail address: Gui-Qiang. Chen@maths.ox.ac.uk

School of Mathematical Sciences, Fudan University, Shanghai 200433; Institute of Applied Mathematics, Academy of Mathematics and Systems Science, Chinese Academy of SciEnCes, Beijing 100190, P. R. China

E-mail address: jkuang12@fudan.edu.cn; jkuang@amss.ac.cn

School of Mathematical Sciences, Fudan University, Shanghai 200433, P. R. China

E-mail address: yongqianz@fudan.edu.cn 